J. Inst. Math. Jussieu (2021), 1-60

doi:10.1017/S1474748021000530 C The Author(s), 2021. Published by Cambridge University Press. 1 This is an Open Access article, distributed under the terms of the Creative Commons Attribution licence (https://creativecommons.org/licenses/by/4.0), which permits unrestricted re-use, distribution and reproduction, provided the original article is properly cited.

\title{
GEOMETRIC WEIGHT-SHIFTING OPERATORS ON HILBERT MODULAR FORMS IN CHARACTERISTIC $p$
}

\author{
FRED DIAMOND (D) \\ Department of Mathematics, King's College London, WC2R 2LS, UK \\ (fred.diamond@kcl.ac.uk)
}

(Received 04 December 2020; revised 18 September 2021; accepted 22 September 2021)

\begin{abstract}
We carry out a thorough study of weight-shifting operators on Hilbert modular forms in characteristic $p$, generalising the author's prior work with Sasaki to the case where $p$ is ramified in the totally real field. In particular, we use the partial Hasse invariants and Kodaira-Spencer filtrations defined by Reduzzi and Xiao to improve on Andreatta and Goren's construction of partial $\Theta$-operators, obtaining ones whose effect on weights is optimal from the point of view of geometric Serre weight conjectures. Furthermore, we describe the kernels of partial $\Theta$-operators in terms of images of geometrically constructed partial Frobenius operators. Finally, we apply our results to prove a partial positivity result for minimal weights of $\bmod p$ Hilbert modular forms.
\end{abstract}

Keywords: Hilbert modular forms, theta operators

2020 Mathematics subject classification: Primary 11F41

Secondary 11F33; 14G35

\section{Introduction}

Thestudy of weight-shifting operations on modular forms has a rich and fruitful history. Besides those naively obtained from the graded algebra structure on the space of classical modular forms of all weights, there is a deeper construction due to Ramanujan [30] which shifts the weight by two using differentiation, leading to a more general theory of Maass-Shimura operators. Analogous weight-shifting operations in characteristic $p$, first studied by Swinnerton-Dyer and Serre [34], take on special significance in the context of congruences between modular forms and the implications for associated Galois representations. In particular, one has the following linear maps from the space of mod $p$ modular forms of weight $k$ and some fixed level $N$ prime to $p$ :

- multiplication by a Hasse invariant $H$, to forms of weight $k+p-1$;

- a differential operator $\Theta$, to forms of weight $k+p+1$;

- a linearised $p$-power map $V$, to forms of weight $p k$.

These maps all have simple descriptions in terms of associated $q$-expansions: if $f$ has $q$-expansion $\sum a_{n} q^{n}$, then that of $H f$ (respectively $\Theta f, V f$ ) is $\sum a_{n} q^{n}$ (respectively $\left.\sum n a_{n} q^{n}, \sum a_{n} q^{p n}\right)$. Following the work of Swinnerton-Dyer and Serre, there were further significant developments to the theory due to Katz [23, 24] (interpreting the constructions more geometrically), Jochnowitz [21, 22] (on the weight filtration and Tate's $\Theta$-cycles) and 
Gross [20] (in the study of companion forms), providing crucial ingredients for Edixhoven's proof of the weight part of Serre's conjecture in [14].

Suppose now that $F$ is a totally real field of degree $d=[F: \mathbb{Q}]$ and consider spaces of Hilbert modular forms of weight $\mathbf{k} \in \mathbb{Z}^{\Sigma}$ and fixed level prime to $p$, where $\Sigma$ denotes the set of embeddings $\left\{\sigma: F \hookrightarrow \overline{\mathbb{Q}}_{p}\right\}$. For such spaces of $p$-adic modular forms, Katz [25] constructed a family of commuting differential operators $\Theta_{\sigma}$, indexed by the $d$ embeddings $\sigma \in \Sigma$. The theory was further developed by Andreatta and Goren [1], who, building on Katz's work and Goren's definition of partial Hasse invariants in $[18,19]$ (if $p$ is unramified in $F$ ), defined partial $\Theta$-operators on spaces of $\bmod p$ Hilbert modular forms.

Under the assumption that $p$ is unramified in $F$, some aspects of the construction of partial $\Theta$-operators in [1] were simplified in [12], which also went on to define partial Frobenius operators (generalising $V$ ) geometrically and use their image to describe kernels of partial $\Theta$-operators. When $p$ is ramified in $F$, the effectiveness of the approach in [1] was limited by the singularities of the available (Deligne-Pappas) model for the Hilbert modular variety. Since then, however, a smooth integral model was constructed by Pappas and Rapoport [29], and the theory of partial Hasse invariants was further developed in this context by Reduzzi and Xiao in [32]. The theory of partial $\Theta$-operators was revisited in that light by Deo, Dimitrov and Wiese in [8], where they closely follow [1]. Here we instead exploit the observations and techniques introduced in [12], applying them directly to the special fibre of the Pappas-Rapoport model to construct and relate partial $\Theta$ and Frobenius operators. In particular, this eliminates extraneous multiples of partial Hasse invariants that appear in [8] and yields results whose implications for minimal weights are motivated by the forthcoming generalisation to the ramified case of the geometric Serre weight conjectures of [12]. The main contributions of this article may be summarised as follows:

- a construction of operators $\Theta_{\tau}$ with optimal effect on weight (Theorem 5.2.1);

- a geometric construction of partial Frobenius operators $V_{\mathfrak{p}}$ (see Subsection 6.2);

- a description of the kernel of $\Theta_{\tau}$ in terms of the image of $V_{\mathfrak{p}}$ (see Subsection 9.1);

- an application to positivity of minimal weights (Theorem 9.2.1).

We should emphasise that the focus of this article is entirely on Hilbert modular forms in characteristic $p$. There is also a rich theory of $\Theta$-operators on $p$-adic automorphic forms which has seen major progress recently in the work of de Shalit and Goren [7] and Eischen, Fintzen et al. [15], which in turn has implications in the characteristic $p$ setting $[6,7,16,17]$. Another advance in characteristic $p$ has been Yamauchi's construction [36] of $\Theta$-operators for mod $p$ Siegel modular forms of degree 2. We remark, however, that all of the work just mentioned only considers automorphic forms on reductive groups which are unramified at $p$; the novelty of this article is largely in the treatment of ramification at $p$.

We now describe the contents in more detail.

We first set out some basic notation and constructions in Subsection 2.1. In particular, we fix a prime $p$, a totally real field $F$ of $\operatorname{degree}^{1} d=[F: \mathbb{Q}]>1$, and let $\mathcal{O}_{F}$ denote the

${ }^{1}$ Including the case $F=\mathbb{Q}$ would introduce different complications in the treatment of cusps and provide no new results. 
ring of integers of $F$ and $S_{p}$ the set of prime ideals of $\mathcal{O}_{F}$ dividing $p$. For each $\mathfrak{p} \in S_{p}$, let $\Sigma_{\mathfrak{p}, 0}$ denote the set of $f_{\mathfrak{p}}$ embeddings $\mathcal{O}_{F} / \mathfrak{p} \rightarrow \overline{\mathbb{F}}_{p}$ and $\Sigma_{\mathfrak{p}}$ the set of $e_{\mathfrak{p}} f_{\mathfrak{p}}$ embeddings $F_{\mathfrak{p}} \rightarrow \overline{\mathbb{Q}}_{p}$, where $f_{\mathfrak{p}}$ (respectively $e_{\mathfrak{p}}$ ) is the residual (respectively ramification) degree of $\mathfrak{p}$. We let

$$
\text { and } \begin{aligned}
\Sigma_{0} & =\coprod_{\mathfrak{p} \in S_{p}} \Sigma_{\mathfrak{p}, 0}=\left\{\tau_{\mathfrak{p}, i} \mid \mathfrak{p} \in S_{p}, i \in \mathbb{Z} / f_{\mathfrak{p}} \mathbb{Z}\right\} \\
\mathrm{\Sigma} & =\coprod_{\mathfrak{p} \in S_{p}} \Sigma_{\mathfrak{p}}=\left\{\theta_{\mathfrak{p}, i, j} \mid \mathfrak{p} \in S_{p}, i \in \mathbb{Z} / f_{\mathfrak{p}} \mathbb{Z}, j=1, \ldots, e_{\mathfrak{p}}\right\},
\end{aligned}
$$

where each $\tau_{\mathfrak{p}, 0} \in \Sigma_{\mathfrak{p}, 0}$ is chosen arbitrarily, and $\tau_{\mathfrak{p}, i}=\tau_{\mathfrak{p}, 0}^{p^{i}}$ and $\theta_{\mathfrak{p}, i, 1}, \ldots, \theta_{\mathfrak{p}, i, e_{\mathfrak{p}}}$ are any ordering of the lifts of $\tau_{\mathfrak{p}, i}$ to $\Sigma_{\mathfrak{p}}$. We also define a 'right-shift' permutation $\sigma$ of $\Sigma$ by

$$
\sigma\left(\theta_{\mathfrak{p}, i, j}\right)= \begin{cases}\theta_{\mathfrak{p}, i, j+1}, & \text { if } j<e_{\mathfrak{p}} \\ \theta_{\mathfrak{p}, i+1,1}, & \text { if } j=e_{\mathfrak{p}}\end{cases}
$$

In Subsection 2.2 we recall the definition of the Pappas-Rapoport model $Y_{U}$ for the Hilbert modular variety of level $U$, where $U$ is any sufficiently small open compact subgroup of $\mathrm{GL}_{2}\left(\mathbb{A}_{F, \mathbf{f}}\right)$ of level prime to $p$. This may be viewed as a coarse moduli space for Hilbert-Blumenthal abelian varieties with additional structure, where this additional structure includes a suitable collection of filtrations on direct summands of its sheaf of invariant differentials. The scheme $Y_{U}$ is then smooth of relative dimension $d$ over $\mathcal{O}$, where $\mathcal{O}$ is the ring of integers of a finite extension of $\mathbb{Q}_{p}$ in $\overline{\mathbb{Q}}_{p}$. Since the main results of the article concern Hilbert modular forms in characteristic $p$, we will restrict our attention to this setting for the remainder of the Introduction and let $\bar{Y}_{U}=Y_{U, \mathbb{F}}$ where $\mathbb{F}$ is the residue field of $\mathcal{O}$.

In Subsections 3.1-3.2 we construct the automorphic line bundles $\overline{\mathcal{A}}_{\mathbf{k}, \mathbf{l}}$ on $\bar{Y}_{U}$ for all $\mathbf{k}, \mathbf{l} \in \mathbb{Z}^{\Sigma}$ and sufficiently small $U$ (of level prime to $p$ ) and define the space of Hilbert modular forms of weight $(\mathbf{k}, \mathbf{l})$ and level $U$ over $\mathbb{F}$ to be

$$
M_{\mathbf{k}, \mathbf{l}}(U ; \mathbb{F})=H^{0}\left(\bar{Y}_{U}, \overline{\mathcal{A}}_{\mathbf{k}, \mathbf{l}}\right) .
$$

The spaces are equipped with a natural Hecke action making

$$
M_{\mathbf{k}, \mathbf{l}}(\mathbb{F}):=\underset{U}{\lim } M_{\mathbf{k}, \mathbf{l}}(U ; \mathbb{F})
$$

a smooth admissible representation of $\mathrm{GL}_{2}\left(\mathbb{A}_{F, \mathbf{f}}\right)$ over $\mathbb{F}$. A key point, as already observed in [12] in the unramified case, is that the parity condition on $\mathbf{k}$ imposed in the definition of Hilbert modular forms in characteristic zero (for the group $\operatorname{Res}_{F / \mathbb{Q}} \mathrm{GL}_{2}$ ) disappears in characteristic $p$. We remark also that the effect of the weight parameter $\mathbf{l}$ (in characteristic $p$ ) is to introduce twists by torsion bundles that make various constructions, in particular, that of partial $\Theta$-operators, compatible with the natural Hecke action.

In Subsections 3.3-4.2 we recall results of Reduzzi and Xiao [32] that will underpin our construction of partial $\Theta$-operators. Firstly, there is a natural Kodaira-Spencer filtration on direct summands of $\Omega_{\bar{Y}_{U} / \mathbb{F}}^{1}$ whose graded pieces are isomorphic to the automorphic line bundles $\overline{\mathcal{A}}_{2 \mathbf{e}_{\theta},-\mathbf{e}_{\theta}}$ (where $\mathbf{e}_{\theta}$ denotes the basis element of $\mathbb{Z}^{\Sigma}$ indexed by $\theta$ ). Secondly, for each $\theta=\theta_{\mathfrak{p}, i, j} \in \Sigma$, there is a partial Hasse invariant

$$
H_{\theta} \in M_{\mathbf{h}_{\theta}, \mathbf{0}}(U ; \mathbb{F}), \quad \text { where } \mathbf{h}_{\theta}=n_{\theta} \mathbf{e}_{\sigma^{-1} \theta}-\mathbf{e}_{\theta}
$$


with $n_{\theta}=p$ if $\theta_{\mathfrak{p}, i, 1}$ for some $\mathfrak{p}, i$, and $n_{\theta}=1$ otherwise. Multiplication by the partial Hasse invariant $H_{\theta}$ thus defines a map

$$
\cdot H_{\theta}: M_{\mathbf{k}, \mathbf{l}}(U ; \mathbb{F}) \longrightarrow M_{\mathbf{k}+\mathbf{h}_{\theta}, \mathbf{l}}(U ; \mathbb{F})
$$

which is easily seen to be Hecke-equivariant. We also analogously define invariants $G_{\theta} \in$ $M_{\mathbf{0}, \mathbf{h}_{\theta}}(U ; \mathbb{F})$ which trivialise the bundles $\overline{\mathcal{A}}_{\mathbf{0}, \mathbf{h}_{\theta}}$. The partial Hasse invariants $H_{\theta}$ refine the ones defined by Andreatta and Goren in [1] and give rise to a natural stratification on $\bar{Y}_{U}$ and a notion of minimal weight $\mathbf{k}_{\min }(f)$ for nonzero $f \in M_{\mathbf{k}, \mathbf{l}}(U ; \mathbb{F})$, which the main result of [10] shows lies in a certain cone $\Xi^{\min } \subset \mathbb{Z}_{>_{0}}^{\Sigma}$ (see (10)).

We then follow the approach of $[12, \S 8]$ to define partial $\Theta$-operators in Section 5 . For each $\tau=\tau_{\mathfrak{p}, i} \in \Sigma_{0}$, this gives a Hecke-equivariant operator

$$
\Theta_{\tau}: M_{\mathbf{k}, \mathbf{l}}(U ; \mathbb{F}) \longrightarrow M_{\mathbf{k}+\mathbf{h}_{\theta}+2 \mathbf{e}_{\theta}, \mathbf{l}-\mathbf{e}_{\theta}}(U ; \mathbb{F})
$$

where $\theta=\theta_{\mathfrak{p}, i, e_{\mathfrak{p}}}$. Note in particular that if $\mathfrak{p}$ is ramified, then the shift ${ }^{2}$ in the weight parameter $\mathbf{k}$ is by $\mathbf{e}_{\sigma^{-1} \theta}+\mathbf{e}_{\theta}$. The idea of the construction, inspired by the one in $[1, \S 12]$, is to divide by fundamental Hasse invariants to get a rational function on the Igusa cover of $\bar{Y}_{U}$, differentiate, project to the top graded piece of the $\tau$-component of the KodairaSpencer filtration and, finally, multiply by fundamental Hasse invariants to descend to $\bar{Y}_{U}$ and eliminate poles. The argument also gives a direct (albeit local) definition of the $\Theta$-operator without reference to the Igusa cover in (12) and establishes the following result (Theorem 5.2.1) generalising [12, Thm. 8.2.2].

Theorem A. Let $\tau=\tau_{\mathfrak{p}, i}$ and $\theta=\theta_{\mathfrak{p}, i, e_{\mathfrak{p}}}$. Then $\Theta_{\tau}(f)$ is divisible by $H_{\theta}$ if and only if either $f$ is divisible by $H_{\theta}$ or $p \mid k_{\theta}$.

We turn to the construction of partial Frobenius operators $V_{\mathfrak{p}}$ in Section 6. This essentially generalises a definition in $[12, \S 9.8]$ but requires significantly more work to actualise if $\mathfrak{p}$ is ramified. We do this using Dieudonné theory to define a partial Frobenius endomorphism $\Phi_{\mathfrak{p}}$ of $\bar{Y}_{U}$ and an isomorphism $\Phi_{\mathfrak{p}}^{*} \overline{\mathcal{A}}_{\mathbf{k}, \mathbf{l}} \cong \overline{\mathcal{A}}_{\mathbf{k}^{\prime \prime}, \mathbf{1}^{\prime \prime}}$, where

$$
\mathbf{k}^{\prime \prime}=\mathbf{k}+\sum_{\theta \in \Sigma_{\mathfrak{p}}} k_{\theta} \mathbf{h}_{\theta} \quad \text { and } \quad \mathbf{l}^{\prime \prime}=\mathbf{l}+\sum_{\theta \in \Sigma_{\mathfrak{p}}} l_{\theta} \mathbf{h}_{\theta},
$$

in order to obtain, for $\mathfrak{p} \in S_{p}$, commuting Hecke-equivariant operators

$$
V_{\mathfrak{p}}: M_{\mathbf{k}, \mathbf{l}}(U ; \mathbb{F}) \longrightarrow M_{\mathbf{k}^{\prime \prime}, \mathbf{l}^{\prime \prime}}(U ; \mathbb{F}) .
$$

We will use $q$-expansions to relate the kernel of $\Theta_{\tau}$ to the image of $V_{\mathfrak{p}}$ for $\tau \in \Sigma_{\mathfrak{p}, 0}$, so we recall the theory in Section 7. This is a straightforward adaptation to our setting of results and methods developed in $[31,4,13,5]$. In Section 8 we compute the (constant) $q$-expansions of the invariants $H_{\theta}$ and $G_{\theta}$ at each cusp of $\bar{Y}_{U}$, and we obtain formulas generalising the classical ones for the effect of the operators $\Theta_{\tau}$ and $V_{\mathfrak{p}}$ on all $q$-expansions. In particular, this shows that the operators $\Theta_{\tau}$ for varying $\tau$ commute.

${ }^{2}$ This precise shift is predictable from the point of view of forthcoming work with Sasaki generalising the geometric Serre weight conjectures of [12] to the ramified case. 
In Subsection 9.1 we turn our attention to the description of the kernel of $\Theta_{\tau}$. The $q$-expansion formulas also show that $\Theta_{\tau} \circ V_{\mathfrak{p}}=0$ if $\tau \in \Sigma_{\mathfrak{p}, 0}$ and that $\operatorname{ker}\left(\Theta_{\tau}\right)$ is the same for all $\tau \in \Sigma_{\mathfrak{p}, 0}$. Theorem A then reduces the study of the kernel to the case of weights of the form $\left(\mathbf{k}^{\prime \prime}, \mathbf{l}^{\prime \prime}\right)$ where $\mathbf{k}^{\prime \prime}, \mathbf{l}^{\prime \prime}$ are as in the definition of $V_{\mathfrak{p}}$, for which the argument proving [12, Thm. 9.8.2] gives the following. ${ }^{3}$

Theorem B. If $\mathbf{k}, \mathbf{l} \in \mathbb{Z}^{\Sigma}$ and $\tau=\tau_{\mathfrak{p}, i}$ and $\theta=\theta_{\mathfrak{p}, i, e_{\mathfrak{p}}}$, then the sequence

$$
0 \longrightarrow M_{\mathbf{k}, \mathbf{l}}(U, \mathbb{F}) \stackrel{V_{\mathfrak{p}}}{\longrightarrow} M_{\mathbf{k}^{\prime \prime}, \mathbf{1}^{\prime \prime}}(U ; \mathbb{F}) \stackrel{\Theta_{\tau}}{\longrightarrow} M_{\mathbf{k}^{\prime \prime}+\mathbf{h}_{\theta}+2 \mathbf{e}_{\theta}, \mathbf{1}^{\prime \prime}-\mathbf{e}_{\theta}}(U ; \mathbb{F})
$$

is exact.

Before discussing the application to positivity of minimal weights, we remark that a less precise relation among the weight-shifting operations can be neatly encapsulated in terms of the algebra of modular forms of all weights

$$
M_{\mathrm{tot}}(U ; \mathbb{F}):=\bigoplus_{\mathbf{k}, \mathbf{l} \in \mathbb{Z}^{\Sigma}} M_{\mathbf{k}, \mathbf{l}}(U ; \mathbb{F}),
$$

or even its direct limit $M_{\text {tot }}(\mathbb{F}):=\lim _{U} M_{\text {tot }}(U ; \mathbb{F}$ ) (over all sufficiently small levels prime to $p$ ). It follows from its definition that the operator $V_{\mathfrak{p}}$ (respectively $\Theta_{\tau}$ ) on the direct sum is an $\mathbb{F}$-algebra endomorphism (respectively $\mathbb{F}$-derivation) of $M_{\text {tot }}(\mathbb{F})$. One also finds that $V_{\mathfrak{p}}$ maps the ideal

$$
\mathfrak{I}=\left\langle H_{\theta}^{\prime}-1, G_{\theta}^{\prime}-1\right\rangle_{\theta \in \Sigma}
$$

to itself. ${ }^{4}$ Furthermore, $\Theta_{\tau}\left(H_{\theta}^{\prime}\right)=\Theta_{\tau}\left(G_{\theta}^{\prime}\right)=0$ for all $\theta \in \Sigma$, so $V_{\mathfrak{p}}$ (respectively $\Theta_{\tau}$ ) induces an $\mathbb{F}$-algebra endomorphism (respectively $\mathbb{F}$-derivation) of the quotient $M_{\text {tot }}(\mathbb{F}) / \mathfrak{I}$. We then have the following consequence of Theorem B (see Theorem 9.3.4).

Theorem C. If $\tau \in \Sigma_{\mathfrak{p}, 0}$, then the sequence

$$
0 \longrightarrow M_{\text {tot }}(\mathbb{F}) / \mathfrak{I} \stackrel{V_{\mathfrak{p}}}{\longrightarrow} M_{\text {tot }}(\mathbb{F}) / \mathfrak{I} \stackrel{\Theta_{\tau}}{\longrightarrow} M_{\text {tot }}(\mathbb{F}) / \mathfrak{I}
$$

is exact.

In Subsection 9.2 we apply our results to refine the main result of [10], which we recall states that minimal weights of nonzero forms always lie in $\Xi^{\text {min }}$. The geometric Serre weight conjectures of [12] (and its forthcoming generalisation to the ramified case) predict that if $f$ is a mod $p$ Hecke eigenform which is non-Eisenstein (in the sense that the associated Galois representation is irreducible), then $\mathbf{k}_{\min }(f)$ should be totally positive. We use Theorems A and B to prove a partial result in this direction (Theorem 9.2.1).

Theorem D. Suppose that $\mathfrak{p} \in S_{p}$ is such that $F_{\mathfrak{p}} \neq \mathbb{Q}_{p}$ and $p^{f_{\mathfrak{p}}}>3$. Suppose that $f \in$ $M_{\mathbf{k}, \mathbf{l}}(U ; \mathbb{F})$ is nonzero and $\mathbf{k}=\mathbf{k}_{\min }(f)$. If $k_{\theta}=0$ for some $\theta \in \Sigma_{\mathfrak{p}}$, then $\mathbf{k}=\mathbf{0}$.

${ }^{3}$ This is a slight reformulation of Theorem 9.1.1.

${ }^{4}$ The $H_{\theta}^{\prime}$ and $G_{\theta}^{\prime}$ are slight modifications of the $H_{\theta}$ and $G_{\theta}$ obtained by rescaling those for which $j=1$; see Subsection 9.3. 
Since the Hecke action on forms of weight $(\mathbf{0}, \mathbf{l})$ is Eisenstein (see Proposition 3.2.2), the theorem implies the total positivity of minimal weights of non-Eisenstein eigenforms in many situations - for example, if $p>3$ and there are no primes $\mathfrak{p} \in S_{p}$ such that $F_{\mathfrak{p}}=\mathbb{Q}_{p}$. We remark that the hypothesis $F_{\mathfrak{p}} \neq \mathbb{Q}_{p}$ cannot be removed from Theorem D: if $\Sigma_{\mathfrak{p}}=\{\theta\}$, then there are nonzero forms whose minimal weight $\mathbf{k}$ satisfies $k_{\theta}=0$ and $k_{\theta^{\prime}}>0$ for some $\theta^{\prime} \neq \theta$. However, forthcoming work with Kassaei will show that the Hecke action on such forms is Eisenstein; like in $[9,10]$, the case of split primes seems to require a completely different method. Unfortunately, the case of $p \leq 3, f_{\mathfrak{p}}=1, e_{\mathfrak{p}}>1$ slips through the crack between the two methods. We do not know whether Theorem D should hold in this case, but we still at least conjecture that the failure is Eisenstein.

\section{Preliminaries}

\subsection{Embeddings and decompositions}

We first set out notation and conventions for various constructions associated to the set of embeddings of a totally real field $F$, which together with a prime $p$ will be fixed throughout the article.

We assume that $F$ has degree $d=[F: \mathbb{Q}]>1$, let $\mathcal{O}_{F}$ denote its ring of integers, $\mathfrak{d}$ its different and $\Sigma$ the set of embeddings $F \rightarrow \overline{\mathbb{Q}}$, where $\overline{\mathbb{Q}}$ is the algebraic closure of $\mathbb{Q}$ in $\mathbb{C}$.

We also fix an embedding $\overline{\mathbb{Q}} \rightarrow \overline{\mathbb{Q}}_{p}$. We let $S_{p}$ denote the set of primes of $\mathcal{O}_{F}$ dividing $p$ and identify $\Sigma$ with $\coprod_{\mathfrak{p} \in S_{p}} \Sigma_{\mathfrak{p}}$ under the natural bijection, where $\Sigma_{\mathfrak{p}}$ denotes the set of embeddings $F_{\mathfrak{p}} \rightarrow \overline{\mathbb{Q}}_{p}$.

For each $\mathfrak{p} \in S_{p}$, we let $F_{\mathfrak{p}, 0}$ denote the maximal unramified subextension of $F_{\mathfrak{p}}$, which we identify with the field of fractions of $W\left(\mathcal{O}_{F} / \mathfrak{p}\right)$. We also let $f_{\mathfrak{p}}$ denote the residue degree $\left[F_{\mathfrak{p}, 0}: \mathbb{Q}_{p}\right], e_{\mathfrak{p}}$ the ramification index $\left[F_{\mathfrak{p}}: F_{\mathfrak{p}, 0}\right]$, and $\Sigma_{\mathfrak{p}, 0}$ the set of embeddings $F_{\mathfrak{p}, 0} \rightarrow$ $\overline{\mathbb{Q}}_{p}$, which we may identify with the set of embeddings $\mathcal{O}_{F} / \mathfrak{p} \rightarrow \overline{\mathbb{F}}_{p}$ or homomorphisms $W\left(\mathcal{O}_{F} / \mathfrak{p}\right) \rightarrow W\left(\overline{\mathbb{F}}_{p}\right)$. For each $\mathfrak{p} \in S_{p}$, we fix a choice of embedding $\tau_{\mathfrak{p}, 0} \in \Sigma_{\mathfrak{p}, 0}$, and for $i \in \mathbb{Z} / f_{\mathfrak{p}} \mathbb{Z}$, we let $\tau_{\mathfrak{p}, i}=\phi^{i} \circ \tau_{\mathfrak{p}, 0}$ where $\phi$ is the Frobenius automorphism of $\overline{\mathbb{F}}_{p}$ (or $W\left(\overline{\mathbb{F}}_{p}\right)$ or its field of fractions), so that $\Sigma_{\mathfrak{p}, 0}=\left\{\tau_{\mathfrak{p}, 1}, \tau_{\mathfrak{p}, 2}, \ldots, \tau_{\mathfrak{p}, f_{\mathfrak{p}}}\right\}$. We also let $\Sigma_{0}=\coprod_{\mathfrak{p} \in S_{p}} \Sigma_{\mathfrak{p}, 0}$. Letting $\mathfrak{q}=\prod_{\mathfrak{p} \in S_{p}} \mathfrak{p}$ denote the radical of $p$ in $\mathcal{O}_{F}$, note that $\Sigma_{0}$ may also be identified with the set of ring homomorphisms $\mathcal{O}_{F} / \mathfrak{q} \rightarrow \overline{\mathbb{F}}_{p}$ (or, indeed, $\mathcal{O}_{F} \rightarrow \overline{\mathbb{F}}_{p}$ ).

For each $\tau=\tau_{\mathfrak{p}, i} \in \Sigma_{0}$, we let $\Sigma_{\tau} \subset \Sigma_{\mathfrak{p}}$ denote the set of embeddings restricting to $\tau$, for which we choose an ordering $\theta_{\mathfrak{p}, i, 1}, \theta_{\mathfrak{p}, i, 2}, \ldots, \theta_{\mathfrak{p}, i, e_{\mathfrak{p}}}$, so that

$$
\Sigma=\coprod_{\tau \in \Sigma_{0}} \Sigma_{\tau}=\left\{\theta_{\mathfrak{p}, i, j} \mid \mathfrak{p} \in S_{p}, i \in \mathbb{Z} / f_{\mathfrak{p}} \mathbb{Z}, 1 \leq j \leq e_{\mathfrak{p}}\right\}
$$

We also define a permutation $\sigma$ of $\Sigma$ whose restriction to each $\Sigma_{\mathfrak{p}}$ is the $e_{\mathfrak{p}} f_{\mathfrak{p}}$-cycle corresponding to the right shift of indices with respect to the lexicographic ordering; that is,

$$
\begin{aligned}
& (1,1) \quad \mapsto \quad(1,2) \quad \mapsto \quad \cdots \quad \mapsto \quad\left(1, e_{\mathfrak{p}}\right) \mapsto \\
& (2,1) \mapsto(2,2) \mapsto \cdots \mapsto\left(2, e_{\mathfrak{p}}\right) \mapsto \\
& \left(f_{\mathfrak{p}}, 1\right) \mapsto\left(f_{\mathfrak{p}}, 2\right) \mapsto \quad \cdots \quad \mapsto \quad\left(f_{\mathfrak{p}}, e_{\mathfrak{p}}\right) \mapsto(1,1)
\end{aligned}
$$


Let $E \subset \overline{\mathbb{Q}}$ be a number field containing the image of $\theta$ for all $\theta \in \Sigma$, let $\mathcal{O}$ be the completion of $\mathcal{O}_{E}$ at the prime determined by the choice of $\overline{\mathbb{Q}} \rightarrow \overline{\mathbb{Q}}_{p}$ and let $\mathbb{F}$ be its residue field. For any $\mathcal{O}_{F, p}=\mathcal{O}_{F} \otimes \mathbb{Z}_{p}$-module $M$, we write $M=\bigoplus_{\mathfrak{p} \in S_{p}} M_{\mathfrak{p}}$ for the decomposition obtained from that of

$$
\mathcal{O}_{F, p} \cong \prod_{\mathfrak{p} \in S_{p}} \mathcal{O}_{F, \mathfrak{p}}
$$

Similarly, for any $W\left(\mathcal{O}_{F} / \mathfrak{p}\right) \otimes_{\mathbb{Z}_{p}} \mathcal{O}$-module $N$, we have a decomposition $N=\bigoplus_{\tau \in \Sigma_{\mathfrak{p}, 0}} N_{\tau}$ obtained from

$$
W\left(\mathcal{O}_{F} / \mathfrak{p}\right) \otimes_{\mathbb{Z}_{p}} \mathcal{O} \cong \prod_{\tau \in \Sigma_{\mathfrak{p}, 0}} \mathcal{O} .
$$

In particular, for any $\mathcal{O}_{F} \otimes \mathcal{O}$-module $M$, we have the decomposition

$$
M=\bigoplus_{\mathfrak{p} \in S_{p}} M_{\mathfrak{p}}=\bigoplus_{\tau \in \Sigma_{0}} M_{\tau},
$$

where we simply write $M_{\tau}$ for $M_{\mathfrak{p}, \tau}$. We also write $M_{\mathfrak{p}, i}$ for $M_{\tau}$ if $\tau=\tau_{\mathfrak{p}, i}$; thus, $M_{\mathfrak{p}, i}$ is the summand of the $\mathcal{O}_{F, \mathfrak{p}} \otimes_{\mathbb{Z}_{p}} \mathcal{O}$-module $M_{\mathfrak{p}}$ on which $W\left(\mathcal{O}_{F} / \mathfrak{p}\right)$ acts via $\tau_{\mathfrak{p}, i}$.

We also fix a choice of uniformiser $\varpi_{\mathfrak{p}}$ for each $\mathfrak{p} \in S_{p}$. We let $f_{\mathfrak{p}}(u)$ denote the minimal polynomial of $\varpi_{\mathfrak{p}}$ over $W\left(\mathcal{O}_{F} / \mathfrak{p}\right)$ and let $f_{\tau}$ denote its image in $\mathcal{O}[u]$ for each $\tau \in \Sigma_{\mathfrak{p}, 0}$; thus, $u \mapsto \varpi_{\mathfrak{p}} \otimes 1$ induces an isomorphism

$$
\mathcal{O}[u] /\left(f_{\tau}(u)\right) \stackrel{\sim}{\longrightarrow} \mathcal{O}_{F, \mathfrak{p}} \otimes_{W\left(\mathcal{O}_{F} / \mathfrak{p}\right), \tau} \mathcal{O}
$$

Furthermore, we have $f_{\tau}(u)=\prod_{\theta \in \Sigma_{\tau}}\left(u-\theta\left(\varpi_{\mathfrak{p}}\right)\right)$, and we define elements

$$
\begin{aligned}
s_{\tau, j} & =\left(u-\theta_{\mathfrak{p}, i, 1}\left(\varpi_{\mathfrak{p}}\right)\right) \cdots\left(u-\theta_{\mathfrak{p}, i, j}\left(\varpi_{\mathfrak{p}}\right)\right) \\
\text { and } t_{\tau, j} & =\left(u-\theta_{\mathfrak{p}, i, j+1}\left(\varpi_{\mathfrak{p}}\right)\right) \cdots\left(u-\theta_{\mathfrak{p}, i, e_{\mathfrak{p}}}\left(\varpi_{\mathfrak{p}}\right)\right)
\end{aligned}
$$

of $\mathcal{O}[u] /\left(f_{\tau}(u)\right)$ for $j=0, \ldots, e_{\mathfrak{p}}$ (with the obvious convention that $s_{\tau, 0}=t_{\tau, e_{\mathfrak{p}}}=1$ ). Note that each of the ideals $\left(s_{\tau, j}\right)$ and $\left(t_{\tau, j}\right)$ is the other's annihilator; furthermore, the quotients of $\mathcal{O}[u] /\left(f_{\tau}(u)\right)$ by these ideals are free over $\mathcal{O}$, and the corresponding ideals in $\mathcal{O}_{F, \mathfrak{p}} \otimes_{W\left(\mathcal{O}_{F} / \mathfrak{p}\right), \tau} \mathcal{O}$ may be described as kernels of projection maps to products of copies of $\mathcal{O}$, and hence depend only on $j$ and the ordering of embeddings and not on the choice of uniformiser $\varpi_{\mathfrak{p}}$.

For an invertible $\mathcal{O}_{F}$-module $L$ and an embedding $\theta=\theta_{\mathfrak{p}, i, j} \in \Sigma_{\tau}$, we define $L_{\theta}$ to be the free rank $1 \mathcal{O}$-module

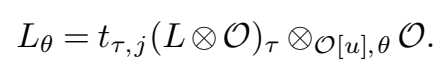

Note that $L_{\theta}$ is not to be identified with $L \otimes_{\mathcal{O}_{F}, \theta} \mathcal{O}$; rather, there is a canonical map $L_{\theta} \rightarrow L \otimes_{\mathcal{O}_{F}, \theta} \mathcal{O}$ which is an isomorphism if and only if $j=e_{\mathfrak{p}}$. If $L$ and $L^{\prime}$ are invertible $\mathcal{O}_{F}$-modules, we will write $L L^{\prime}$ for $L \otimes_{\mathcal{O}_{F}} L^{\prime}$ and $L^{-1}$ for $\operatorname{Hom}_{\mathcal{O}_{F}}\left(L, \mathcal{O}_{F}\right)$. Note that there are natural maps $L_{\theta} \otimes_{\mathcal{O}} L_{\theta}^{\prime} \rightarrow\left(L L^{\prime}\right)_{\theta}$ and $\left(L^{-1}\right)_{\theta} \rightarrow \operatorname{Hom}_{\mathcal{O}}\left(L_{\theta}, \mathcal{O}\right)$ but, again, these are isomorphisms if and only if $j=e_{\mathfrak{p}}$. 


\subsection{Pappas-Rapoport models}

In this section we recall the description of the Hilbert modular variety as a coarse moduli space for abelian varieties with additional structure, along with the construction by Pappas and Rapoport of a smooth integral model (see [29] and [33]).

Let $G=\operatorname{Res}_{F / \mathbb{Q}} \mathrm{GL}_{2}$ and let $U$ be an open compact subgroup of $\mathrm{GL}_{2}\left(\widehat{\mathcal{O}}_{F}\right) \subset$ $\mathrm{GL}_{2}\left(\mathbb{A}_{F, \mathbf{f}}\right)=G\left(\mathbb{A}_{\mathbf{f}}\right)$ of the form $U_{p} U^{p}$, where $U_{p}=\mathrm{GL}_{2}\left(\mathcal{O}_{F, p}\right)$ and $U^{p} \subset \mathrm{GL}_{2}\left(\mathbb{A}_{F, \mathbf{f}}^{(p)}\right)$ is sufficiently small, in a sense to be specified below.

We consider the functor which associates, to a locally Noetherian $\mathcal{O}$-scheme $S$, the set of isomorphism classes of data $\left(A, \iota, \lambda, \eta, \mathcal{F}^{\bullet}\right)$, where:

- $s: A \rightarrow S$ is an abelian scheme of relative dimension $d$;

- $\iota: \mathcal{O}_{F} \rightarrow \operatorname{End}_{S}(A)$ is an embedding such that $\left(s_{*} \Omega_{A / S}^{1}\right)_{\mathfrak{p}}$ is, locally on $S$, free of rank $e_{\mathfrak{p}}$ over $W\left(\mathcal{O}_{F} / \mathfrak{p}\right) \otimes_{\mathbb{Z}_{p}} \mathcal{O}_{S}$ for each $\mathfrak{p} \in S_{p}$

- $\lambda$ is an $\mathcal{O}_{F}$-linear quasi-polarisation of $A$ such that for each connected component $S_{i}$ of $S, \lambda$ induces an isomorphism $\mathfrak{c}_{i} \mathfrak{d} \otimes_{\mathcal{O}_{F}} A_{S_{i}} \rightarrow A_{S_{i}}^{\vee}$ for some fractional ideal $\mathfrak{c}_{i}$ of $F$ prime to $p$;

- $\quad \eta$ is a level $U^{p}$ structure on $A$; that is, for a choice of geometric point $\bar{s}_{i}$ on each connected component $S_{i}$ of $S$, the data of a $\pi_{1}\left(S_{i}, \bar{s}_{i}\right)$-invariant $U^{p}$-orbit of $\widehat{\mathcal{O}}_{F}^{(p)}=\mathcal{O}_{F} \otimes \widehat{\mathbb{Z}}^{(p)}$-linear isomorphisms ${ }^{5}$

$$
\eta_{i}:\left(\widehat{\mathcal{O}}_{F}^{(p)}\right)^{2} \rightarrow \mathfrak{d} \otimes_{\mathcal{O}_{F}} T^{(p)}\left(A_{\bar{s}_{i}}\right),
$$

where $T^{(p)}$ denotes the product over $\ell \neq p$ of the $\ell$-adic Tate modules and $g \in U^{p}$ acts on $\eta_{i}$ by pre-composing with right multiplication by $g^{-1}$;

- $\mathcal{F}^{\bullet}$ is a collection of Pappas-Rapoport filtrations; that is, for each $\tau=\tau_{\mathfrak{p}, i} \in \Sigma_{0}$, an increasing filtration of $\mathcal{O}_{F, \mathfrak{p}} \otimes_{W\left(\mathcal{O}_{F} / \mathfrak{p}\right), \tau} \mathcal{O}_{S}$-modules

$$
0=\mathcal{F}_{\tau}^{(0)} \subset \mathcal{F}_{\tau}^{(1)} \subset \cdots \subset \mathcal{F}_{\tau}^{\left(e_{\mathfrak{p}}-1\right)} \subset \mathcal{F}_{\tau}^{\left(e_{\mathfrak{p}}\right)}=\left(s_{*} \Omega_{A / S}^{1}\right)_{\tau}
$$

such that for $j=1, \ldots, e_{\mathfrak{p}}$, the quotient

$$
\mathcal{L}_{\mathfrak{p}, i, j}:=\mathcal{F}_{\tau}^{(j)} / \mathcal{F}_{\tau}^{(j-1)}
$$

is a line bundle on $S$ on which $\mathcal{O}_{F}$ acts via $\theta_{\mathfrak{p}, i, j}$.

The proof of [12, Lemma 2.4.1] does not assume $p$ is unramified in $F$ and shows that if $U^{p}$ is sufficiently small and $\alpha$ is an automorphism of a triple $(A, \iota, \eta)$ over a connected scheme $S$, then $\alpha=\iota(\mu)$ for some $\mu \in U \cap \mathcal{O}_{F}^{\times}$. If we assume further that $-1 \notin U \cap \mathcal{O}_{F}^{\times}$, then it follows from standard arguments that the functor above is representable by an infinite disjoint union of quasi-projective schemes over $\mathcal{O}$, which we denote by $\widetilde{Y}_{U}$, and the argument in the proof of [33, Prop. 6] shows that $\widetilde{Y}_{U}$ is smooth of relative dimension $d$ over $\mathcal{O}$. Furthermore, defining an action of $\mathcal{O}_{F,(p),+}^{\times}$on $\widetilde{Y}_{U}$ by

$$
\nu \cdot\left(A, \iota, \lambda, \eta, \mathcal{F}^{\bullet}\right)=\left(A, \iota, \nu \lambda, \eta, \mathcal{F}^{\bullet}\right)
$$

\footnotetext{
${ }^{5}$ Note the conventions in place with respect to the different, which are motivated by the point of view that we wish to systematically trivialise modules defined by cohomological constructions.
} 
(as in $[11, \S 2.1 .3]$ ), we see that the resulting action of $\mathcal{O}_{F,(p),+}^{\times} /\left(U \cap \mathcal{O}_{F}^{\times}\right)^{2}$ is free and the quotient is representable by a smooth quasi-projective scheme over $\mathcal{O}$, which we denote by $Y_{U}$.

We also have a natural right action of $\mathrm{GL}_{2}\left(\mathbb{A}_{F, \mathbf{f}}^{(p)}\right)$ on the inverse system of schemes $Y_{U}$ induced by pre-composing the level structure $\eta$ with right multiplication by $g^{-1}$. More precisely, suppose that $U_{1}$ and $U_{2}$ are as above (with $U_{1}^{p}$ and $U_{2}^{p}$ sufficiently small) and $g \in \mathrm{GL}_{2}\left(\mathbb{A}_{F, \mathbf{f}}^{(p)}\right)$ is such that $g^{-1} U_{1} g \subset U_{2}$. Letting $\left(A, \iota, \lambda, \eta, \mathcal{F}^{\bullet}\right)$ denote the universal object over $\widetilde{Y}_{U_{1}}$, there is a prime-to- $p$ quasi-isogeny $A \rightarrow A^{\prime}$ of abelian varieties with $\mathcal{O}_{F}$-action inducing isomorphisms $\mathfrak{d} \otimes_{\mathcal{O}_{F}} T^{(p)}\left(A_{\bar{s}_{i}}^{\prime}\right) \cong \eta_{i}\left(\left(\widehat{\mathcal{O}}_{F}^{(p)}\right)^{2} g^{-1}\right)$ for each $i$, from which we obtain a level $U_{2}$-structure $\eta^{\prime}=\eta \circ r_{g^{-1}}$ on $A^{\prime}$ (where $r_{g^{-1}}$ denotes right multiplication by $g^{-1}$ ). Together with the other data inherited from $A$, we obtain an object $\left(A^{\prime}, \iota^{\prime}, \lambda^{\prime}, \eta^{\prime}, \mathcal{F}^{\prime \bullet}\right)$ corresponding to a morphism $\widetilde{\rho}_{g}: \widetilde{Y}_{U_{1}} \rightarrow \widetilde{Y}_{U_{2}}$ and descending to a morphism $Y_{U_{1}} \rightarrow Y_{U_{2}}$ which we denote $\rho_{g}$. These morphisms satisfy the evident compatibility $\rho_{g_{2}} \circ \rho_{g_{1}}=\rho_{g_{1} g_{2}}$ whenever $g_{1}^{-1} U_{1} g_{1} \subset U_{2}$ and $g_{2}^{-1} U_{2} g_{2} \subset U_{3}$.

Finally, we remark that the schemes $Y_{U}$ define smooth integral models over $\mathcal{O}$ for the Hilbert modular varieties associated to the group $G$ (with the usual choice of Shimura datum), and their generic fibres and resulting $\mathrm{GL}_{2}\left(\mathbb{A}_{F, \mathbf{f}}^{(p)}\right)$-action may be identified with those obtained from a system of canonical models. In particular, for any $\mathcal{O} \rightarrow \mathbb{C}$, we have isomorphisms

$$
Y_{U}(\mathbb{C}) \cong \mathrm{GL}_{2}(F)_{+} \backslash\left(\mathfrak{H}^{\Sigma} \times \mathrm{GL}_{2}\left(\mathbb{A}_{F, \mathbf{f}}\right) / U\right) \cong \mathrm{GL}_{2}\left(\mathcal{O}_{F,(p)}\right)_{+} \backslash\left(\mathfrak{H}^{\Sigma} \times \mathrm{GL}_{2}\left(\mathbb{A}_{F, \mathbf{f}}^{(p)}\right) / U^{p}\right)
$$

compatible with the right action of $\mathrm{GL}_{2}\left(\mathbb{A}_{F, \mathbf{f}}^{(p)}\right)$ on the inverse system and inducing a bijection between the set of geometric components of $Y_{U}$ and

$$
\mathbb{A}_{F, \mathbf{f}}^{\times} / F_{+}^{\times} \operatorname{det}(U) \cong\left(\mathbb{A}_{F, \mathbf{f}}^{(p)}\right)^{\times} / \mathcal{O}_{F,(p),+}^{\times} \operatorname{det}\left(U^{p}\right)
$$

These isomorphisms arise in turn from ones of the form

$$
\widetilde{Y}_{U}(\mathbb{C}) \cong \mathrm{SL}_{2}\left(\mathcal{O}_{F,(p)}\right) \backslash\left(\mathfrak{H}^{\Sigma} \times \mathrm{GL}_{2}\left(\mathbb{A}_{F, \mathbf{f}}^{(p)}\right) / U^{p}\right)
$$

under which the set of geometric components of $\widetilde{Y}_{U}$ is described by $\left(\mathbb{A}_{F, \mathbf{f}}^{(p)}\right)^{\times} / \operatorname{det}\left(U^{p}\right)$.

\section{Automorphic bundles}

\subsection{Pairings and duality}

Before introducing the line bundles whose sections define the automorphic forms of interest in the article, we present a plethora of perfect pairings provided by Poincaré duality.

We fix a sufficiently small $U$ as in Subsection 2.2 and consider the de Rham cohomology sheaves $\mathcal{H}_{\mathrm{dR}}^{1}(A / S)=\mathbb{R}^{1} s_{*} \Omega_{A / S}^{\bullet}$ on the universal abelian scheme $A$ over $S=\widetilde{Y}_{U}$. Recall 
that these sheaves are locally free of rank 2 over $\mathcal{O}_{F} \otimes \mathcal{O}_{S}$. Furthermore, Poincaré duality and the polarisation $\lambda$ induce an $\mathcal{O}_{F} \otimes \mathcal{O}_{S}$-linear isomorphism

$$
\begin{aligned}
& \left.\mathcal{H}_{\mathrm{dR}}^{1}(A / S) \stackrel{\sim}{\longrightarrow} \mathcal{H}^{\circ} m_{\mathcal{O}_{S}}\left(\mathcal{H}_{\mathrm{dR}}^{1}\left(A^{\vee} / S\right), \mathcal{O}_{S}\right) \stackrel{\sim}{\longleftarrow} \mathcal{H}^{\circ} m_{\mathcal{O}_{S}}\left(\mathcal{H}_{\mathrm{dR}}^{1}\left(\mathfrak{c d} \otimes_{\mathcal{O}_{F}} A\right) / S\right), \mathcal{O}_{S}\right) \\
& 2 \| \\
& \mathcal{H o m}_{\mathcal{O}_{S}}\left(\mathfrak{d}^{-1} \otimes_{\mathcal{O}_{F}} \mathcal{H}_{\mathrm{dR}}^{1}(A / S), \mathcal{O}_{S}\right)
\end{aligned}
$$

(where $\mathfrak{c}$ depends on the connected component of $S$ and disappears from the last expression since it is prime to $p)$. This in turn induces $\mathcal{O}_{F, \mathfrak{p}} \otimes_{W\left(\mathcal{O}_{F} / \mathfrak{p}\right), \tau} \mathcal{O}_{S}$-linear isomorphisms

$$
\mathcal{H}_{\mathrm{dR}}^{1}(A / S)_{\tau} \cong \mathcal{H}^{\circ} m_{\mathcal{O}_{S}}\left(\mathfrak{d}_{\mathfrak{p}}^{-1} \otimes_{\mathcal{O}_{F, \mathfrak{p}}} \mathcal{H}_{\mathrm{dR}}^{1}(A / S)_{\tau}, \mathcal{O}_{S}\right)
$$

which we view as defining a perfect $\mathcal{O}_{S}$-bilinear pairing $\langle\cdot, \cdot\rangle_{\tau}^{0}$ between $\mathcal{H}_{\tau}:=\mathcal{H}_{\mathrm{dR}}^{1}(A / S)_{\tau}$ and $\mathfrak{d}_{\mathfrak{p}}^{-1} \otimes_{\mathcal{O}_{F, \mathfrak{p}}} \mathcal{H}_{\tau}$ for $\tau \in \Sigma_{\mathfrak{p}, 0}$. Furthermore, the pairing is alternating in the sense that $\langle x, c \otimes y\rangle_{\tau}^{0}=-\langle y, c \otimes x\rangle_{\tau}^{0}$ on sections. Alternatively, we may apply the canonical $\mathcal{O}_{F} \otimes R$ linear isomorphism

$$
\operatorname{Hom}_{\mathcal{O}_{F} \otimes R}\left(M, \mathcal{O}_{F} \otimes R\right) \stackrel{\sim}{\longrightarrow} \operatorname{Hom}_{R}\left(\mathfrak{d}^{-1} \otimes \mathcal{O}_{F} M, R\right)
$$

induced by the trace for any $\mathcal{O}_{F} \otimes R$-module $M$ to obtain an $\mathcal{O}_{F} \otimes \mathcal{O}_{S}$-linear isomorphism

$$
\mathcal{H}_{\mathrm{dR}}^{1}(A / S) \stackrel{\sim}{\longrightarrow} \mathcal{H o m}_{\mathcal{O}_{F} \otimes \mathcal{O}_{S}}\left(\mathfrak{c}^{-1} \otimes \mathcal{O}_{F} \mathcal{H}_{\mathrm{dR}}^{1}(A / S), \mathcal{O}_{F} \otimes \mathcal{O}_{S}\right),
$$

and hence a perfect alternating $\mathcal{O}_{F, \mathfrak{p}} \otimes_{W\left(\mathcal{O}_{F} / \mathfrak{p}\right), \tau} \mathcal{O}_{S^{-}}$-bilinear pairing $\langle\cdot, \cdot\rangle_{\tau}$ on $\mathcal{H}_{\tau}$.

Note that $\mathcal{H}_{\tau}$ is locally free of rank 2 over $\mathcal{O}_{F, \mathfrak{p}} \otimes_{W\left(\mathcal{O}_{F} / \mathfrak{p}\right), \tau} \mathcal{O}_{S}$ and hence a vector bundle of rank $2 e_{\mathfrak{p}}$ over $\mathcal{O}_{S}$. Furthermore, $\left(s_{*} \Omega_{A / S}^{1}\right)_{\tau}$ is a subbundle of $\mathcal{H}_{\tau}$ of rank $e_{\mathfrak{p}}$ but is not locally free over $\mathcal{O}_{F, \mathfrak{p}} \otimes_{W\left(\mathcal{O}_{F} / \mathfrak{p}\right), \tau} \mathcal{O}_{S}$ if $e_{\mathfrak{p}}>1$ (in which case the failure is on a closed subscheme of codimension 1) and, more generally, $\mathcal{F}_{\tau}^{(j)}$ is a subbundle of rank $j$ for $j=0,1, \ldots, e_{\mathfrak{p}}$.

Recall that for $j=0,1, \ldots, e_{\mathfrak{p}}$, we defined (see (1)) elements $s_{\tau, j}$ and $t_{\tau, j}$ of the ring $\mathcal{O}_{F, \mathfrak{p}} \otimes_{W\left(\mathcal{O}_{F} / \mathfrak{p}\right), \tau} \mathcal{O} \cong \mathcal{O}[u] /\left(f_{\tau}(u)\right)$, where $f_{\tau}$ is the image under $\tau$ of the Eisenstein polynomial associated to our choice of uniformiser $\varpi_{\mathfrak{p}}$. In the following, we shall fix $\tau$ and omit the subscripts $\tau, \mathfrak{p}$ and $i$ to disencumber the notation; we also write simply $W$ for $W\left(\mathcal{O}_{F} / \mathfrak{p}\right)$. Note that since $\mathcal{H}$ is locally free over $\mathcal{O}_{F, \mathfrak{p}} \otimes_{W} \mathcal{O}_{S}$ and $\mathcal{F}^{(j)}$ is annihilated by $s_{j}$, we have that $\mathcal{F}^{(j)}$ is in fact a subbundle of $t_{j} \mathcal{H}$.

For a subsheaf $\mathcal{E} \subset \mathcal{H}$ of $\mathcal{O}_{F, \mathfrak{p}} \otimes_{W} \mathcal{O}_{S}$-submodules, we define $\mathcal{E}^{\perp}$ to be its orthogonal complement under the pairing $\langle\cdot, \cdot\rangle$; that is, the kernel of the morphism

$$
\mathcal{H} \stackrel{\sim}{\longrightarrow} \mathcal{H o m}_{\mathcal{O}_{F, \mathfrak{p}} \otimes_{W} \mathcal{O}_{S}}\left(\mathcal{H}, \mathcal{O}_{F, \mathfrak{p}} \otimes_{W} \mathcal{O}_{S}\right) \longrightarrow \mathcal{H o m}_{\mathcal{O}_{F, \mathfrak{p}} \otimes_{W} \mathcal{O}_{S}}\left(\mathcal{E}, \mathcal{O}_{F, \mathfrak{p}} \otimes_{W} \mathcal{O}_{S}\right)
$$

or, equivalently, the orthogonal complement of $\mathfrak{d}_{\mathfrak{p}}^{-1} \otimes_{\mathcal{O}_{F, \mathfrak{p}}} \mathcal{E}$ under the pairing $\langle\cdot, \cdot\rangle^{0}$. Note from the latter description that if $\mathcal{E}$ is an $\mathcal{O}_{S}$-subbundle of $\mathcal{H}$, then so is $\mathcal{E}^{\perp}$.

Lemma 3.1.1. We have the equality $\left(\mathcal{F}^{(j)}\right)^{\perp}=t_{j}^{-1} \mathcal{F}^{(j)}$ for $j=0,1, \ldots$, e, where $t_{j}^{-1} \mathcal{F}^{(j)}=$ $\operatorname{ker}\left(\mathcal{H} \stackrel{t_{j}}{\longrightarrow} t_{j} \mathcal{H} \longrightarrow t_{j} \mathcal{H} / \mathcal{F}^{(j)}\right)$ is the pre-image sheaf of $\mathcal{F}^{(j)}$ under $t_{j}$.

Proof. We prove the lemma by induction on $j$, the case of $j=0$ being obvious. 
Suppose then that $1 \leq j \leq e$ and that $\left(\mathcal{F}^{(j-1)}\right)^{\perp}=t_{j-1}^{-1} \mathcal{F}^{(j-1)}$. Note that $\left(\mathcal{F}^{(j)}\right)^{\perp}$ and $t_{j}^{-1} \mathcal{F}^{(j)}$ are both kernels of surjective morphisms from $\mathcal{H}$ to vector bundles of rank $j$ on $S$, so each is a subbundle of rank $2 e-j$, and hence it suffices to prove the inclusion $t_{j}^{-1} \mathcal{F}^{(j)} \subset\left(\mathcal{F}^{(j)}\right)^{\perp}$. To do so, we may work locally on $S$ and assume that $\mathcal{F}^{(j)}(V)=$ $\mathcal{F}^{(j-1)}(V) \oplus R x_{j}$ where $V=\operatorname{Spec} R$ is a Noetherian open subscheme of $\widetilde{Y}_{U}$ such that $\left.\mathcal{H}\right|_{V}$ is free over $\mathcal{O}_{F, \mathfrak{p}} \otimes_{W} \mathcal{O}_{V}$ and $x_{j} \in \mathcal{H}(V)$ satisfies $\left(u-\theta_{j}(\varpi)\right) x_{j} \in \mathcal{F}^{(j-1)}(V)$. In particular, $x_{j}=t_{j} y_{j}$ for some $y_{j} \in t_{j-1}^{-1} \mathcal{F}^{(j-1)}(V)$, so that

$$
t_{j}^{-1} \mathcal{F}^{(j)}(V)=t_{j}^{-1} \mathcal{F}^{(j-1)}(V) \oplus R y_{j} \quad \text { and } \quad\left(\mathcal{F}^{(j)}(V)\right)^{\perp}=\left(\mathcal{F}^{(j-1)}(V)\right)^{\perp} \cap\left(R x_{j}\right)^{\perp} .
$$

Note that if $w \in t_{j}^{-1} \mathcal{F}^{(j)}(V)$, then

$$
t_{j-1} w=\left(u-\theta_{j}(\varpi)\right) t_{j} w \in\left(u-\theta_{j}(\varpi)\right) \mathcal{F}^{(j)}(V) \subset \mathcal{F}^{(j-1)}(V),
$$

so that $\left.w \in t_{j-1}^{-1} \mathcal{F}^{(j-1)}(V)=\left(\mathcal{F}^{(j-1)}\right)(V)\right)^{\perp}$. Furthermore, if $w \in t_{j}^{-1} \mathcal{F}^{(j-1)}(V)$, then

$$
\left\langle w, x_{j}\right\rangle=\left\langle w, t_{j} y_{j}\right\rangle=\left\langle t_{j} w, y_{j}\right\rangle
$$

since $t_{j} w \in \mathcal{F}^{(j-1)}$ and $y_{j} \in t_{j-1}^{-1} \mathcal{F}^{(j-1)}(V)=\left(\mathcal{F}^{(j-1)}(V)\right)^{\perp}$. Finally, since the pairing is alternating, we have

$$
\left\langle y_{j}, x_{j}\right\rangle=\left\langle y_{j}, t_{j} y_{j}\right\rangle=\left\langle t_{j} y_{j}, y_{j}\right\rangle=\left\langle x_{j}, y_{j}\right\rangle=-\left\langle y_{j}, x_{j}\right\rangle
$$

which implies that $\left\langle y_{j}, x_{j}\right\rangle=0$ (since $\widetilde{Y}_{U}$ is flat over $\mathbb{Z}_{2}$ if $p=2$ ).

We have now shown that $t_{j}^{-1} \mathcal{F}^{(j)}(V) \subset\left(\mathcal{F}^{(j-1)}(V)\right)^{\perp} \cap\left(R x_{j}\right)^{\perp}=\left(\mathcal{F}^{(j)}(V)\right)^{\perp}$, as required.

We now define $\mathcal{G}^{(j)}=\left(u-\theta_{j}(\varpi)\right)^{-1} \mathcal{F}^{(j-1)}$ for $j=1, \ldots, e$. Thus, $\mathcal{G}^{(j)}$ is a rank $j+1$ subbundle of $\mathcal{H}$, and we have inclusions of subbundles $\mathcal{F}^{(j-1)} \subset \mathcal{F}^{(j)} \subset \mathcal{G}^{(j)}$, so that $\mathcal{L}_{j}:=\mathcal{F}^{(j)} / \mathcal{F}^{(j-1)}$ is a rank 1 subbundle of the rank 2 vector bundle $\mathcal{P}_{j}:=\mathcal{G}^{(j)} / \mathcal{F}^{(j-1)}$. Furthermore, all of the inclusions are morphisms of $\mathcal{O}_{F, \mathfrak{p}} \otimes_{W} \mathcal{O}_{S}$-modules, and $\mathcal{O}_{F}$ acts on $\mathcal{P}_{j}$ via $\theta_{j}$.

Note that $\mathcal{G}^{(j)}$ is annihilated by $s_{j}$, so that $\mathcal{G}^{(j)} \subset t_{j} \mathcal{H}$, and we have $t_{j}^{-1} \mathcal{G}^{(j)}=$ $t_{j-1}^{-1} \mathcal{F}^{(j-1)}=\left(\mathcal{F}^{j-1}\right)^{\perp}$ by Lemma 3.1.1, from which it follows also that

$$
t_{j}^{-1} \mathcal{F}^{(j-1)}=t_{j}^{-1}\left(t_{j}^{-1} \mathcal{G}^{(j)}\right)^{\perp}=\left(\mathcal{G}^{(j)}\right)^{\perp} .
$$

(The last equality can be seen by arguing locally on sections or by noting that the diagram

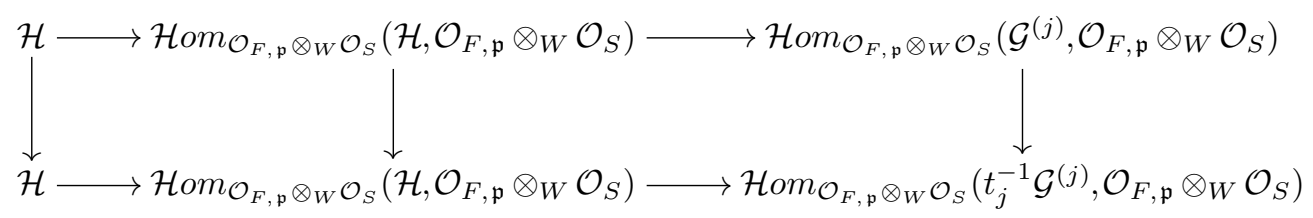

commutes, where the left horizontal morphisms are defined by the pairing, the right by restriction and all of the vertical morphisms by $t_{j}$. The kernel of the composite along the top is $\left(\mathcal{G}^{(j)}\right)^{\perp}$, whereas $t_{j}^{-1}\left(t_{j}^{-1} \mathcal{G}^{(j)}\right)^{\perp}$ is the kernel of the composite along the left and bottom. Since $t_{j}: t_{j}^{-1} \mathcal{G}^{(j)} \rightarrow \mathcal{G}^{(j)}$ is a surjective morphism of vector bundles, the 
leftmost vertical arrow is injective, so these kernels coincide.) Therefore, multiplication by $t_{j}$ defines an isomorphism $\left(\mathcal{F}^{(j-1)}\right)^{\perp} /\left(\mathcal{G}^{(j)}\right)^{\perp} \stackrel{\sim}{\longrightarrow} \mathcal{G}^{(j)} / \mathcal{F}^{(j-1)}$, and composing its inverse with the isomorphism

$$
\left(\mathcal{F}^{(j-1)}\right)^{\perp} /\left(\mathcal{G}^{(j)}\right)^{\perp} \stackrel{\sim}{\longrightarrow} \mathcal{H o m}_{\mathcal{O}_{F, \mathfrak{p}} \otimes_{W} \mathcal{O}_{S}}\left(\mathcal{G}^{(j)} / \mathcal{F}^{(j-1)}, \mathcal{O}_{F, \mathfrak{p}} \otimes_{W} \mathcal{O}_{S}\right)
$$

induced by the pairing on $\mathcal{H}$, we obtain an alternating $\left(\mathcal{O}_{F, \mathfrak{p}} \otimes_{W} \mathcal{O}_{S}\right)$-valued pairing $\langle\cdot, \cdot\rangle_{j}$ on $\mathcal{P}_{j}:=\mathcal{G}^{(j)} / \mathcal{F}^{(j-1)}$, whose description on sections is given in terms of (4) by

$$
\left\langle t_{j} x, t_{j} y\right\rangle_{j}=\left\langle x, t_{j} y\right\rangle=\left\langle t_{j} x, y\right\rangle .
$$

Note that since $\mathcal{O}_{F, \mathfrak{p}}$ acts via $\tau_{j}$ on $\mathcal{P}_{j}$, we in fact have the identification

$$
\mathcal{H} o m_{\mathcal{O}_{F, \mathfrak{p}} \otimes_{W} \mathcal{O}_{S}}\left(\mathcal{P}_{j}, \mathcal{O}_{F, \mathfrak{p}} \otimes_{W} \mathcal{O}_{S}\right)=\mathcal{H} o m_{\mathcal{O}_{S}}\left(\mathcal{P}_{j}, \mathcal{I}_{j}\right)
$$

where $\mathcal{I}_{j}$ is the sheaf of ideals, and trivial rank $1 \mathcal{O}_{S}$-subbundle, of $\mathcal{O}_{F, \mathfrak{p}} \otimes_{W} \mathcal{O}_{S}$ generated by the global section $\prod_{j^{\prime} \neq j}\left(u-\tau_{j^{\prime}}\left(\varpi_{\mathfrak{p}}\right)\right)$. We thus obtain a trivialisation of $\wedge_{\mathcal{O}_{S}}^{2} \mathcal{P}_{j}$ corresponding to a perfect $\mathcal{O}_{S}$-valued pairing $\langle\cdot, \cdot\rangle_{j}^{0}$, which an unravelling of definitions shows is given in terms of the original pairing $\langle\cdot, \cdot\rangle^{0}$ of (3) by the formula

$$
\left\langle t_{j} x, y\right\rangle_{j}^{0}=\left\langle x, f^{\prime}\left(\varpi_{\pi_{\mathfrak{p}}}\right)^{-1} \otimes y\right\rangle^{0}
$$

on sections (where $f^{\prime}$ is the derivative of the Eisenstein polynomial $f$ ).

\subsection{Automorphic line bundles}

Recall that the Pappas-Rapoport model $\tilde{Y}_{U}$ is equipped with line bundles ${ }^{6} \mathcal{L}_{j}$, which we described in Subsection 3.1 as subbundles of the rank 2 vector bundles $\mathcal{P}_{j}$. It is natural and convenient to consider also the twists of $\mathcal{L}_{j}$ by powers of the determinant bundle of $\mathcal{P}_{j}$ :

$$
\mathcal{N}_{j}=\wedge_{\mathcal{O}_{S}}^{2} \mathcal{P}_{j} \cong \mathcal{L}_{j} \otimes_{\mathcal{O}_{S}} \mathcal{M}_{j}
$$

where $\mathcal{M}_{j}$ is the line bundle $\mathcal{P}_{j} / \mathcal{L}_{j}$. Note that the pairing $\langle\cdot, \cdot\rangle_{j}^{0}$ defines an isomorphism $\mathcal{M}_{j} \stackrel{\sim}{\longrightarrow} \mathcal{L}_{j}^{-1}$ and a trivialisation $\mathcal{O}_{S} \stackrel{\sim}{\longrightarrow} \mathcal{N}_{j}$ (which depends on the choice of $\varpi$ ).

As we will now consider these bundles for varying $\tau$, we resume writing the indicative subscripts; thus, for $\tau=\tau_{\mathfrak{p}, i}$, we will denote $\mathcal{G}^{(j)}$ by $\mathcal{G}_{\tau}^{(j)}, \mathcal{P}_{j}$ by $\mathcal{P}_{\mathfrak{p}, i, j}$ and similarly for $\mathcal{M}_{j}$ and $\mathcal{N}_{j}$. We also freely replace the subscript ' $\mathfrak{p}, i, j$ ' by $\theta$, where $\theta=\theta_{\mathfrak{p}, i, j}$, so that for each $\theta \in \Sigma$, we have now defined a rank 2 vector bundle $\mathcal{P}_{\theta}$ and line bundles $\mathcal{L}_{\theta}, \mathcal{M}_{\theta}, \mathcal{N}_{\theta}$ on $S=\widetilde{Y}_{U}$, along with exact sequences

$$
0 \longrightarrow \mathcal{L}_{\theta} \longrightarrow \mathcal{P}_{\theta} \longrightarrow \mathcal{M}_{\theta} \longrightarrow 0
$$

and a trivialisation of $\mathcal{N}_{\theta}=\wedge_{\mathcal{O}_{S}}^{2} \mathcal{P}_{\theta} \cong \mathcal{L}_{\theta} \otimes_{\mathcal{O}_{S}} \mathcal{M}_{\theta}$. Furthermore, the bundles $\mathcal{P}_{\theta}, \mathcal{L}_{\theta}$ and $\mathcal{M}_{\theta}$ are $\mathcal{O}_{F} \otimes \mathcal{O}_{S}$-subquotients of $\mathcal{H}_{\mathrm{dR}}^{1}(A / S)$ on which $\mathcal{O}_{F}$ acts via $\theta$.

Recall that we have an action of $\mathcal{O}_{F,(p),+}^{\times}$on $\widetilde{Y}_{U}$ defined by multiplication on the quasipolarisation. In particular, if $\nu \in \mathcal{O}_{F,(p),+}^{\times}$, then the identification of $\nu^{*} A$ with $A$ induces an $\mathcal{O}_{F} \otimes \mathcal{O}_{S}$-linear isomorphism $\nu^{*} \mathcal{H}_{\mathrm{dR}}^{1}(A / S) \cong \mathcal{H}_{\mathrm{dR}}^{1}(A / S)$ under which $\nu^{*} \mathcal{F}^{\bullet}$ corresponds to

${ }^{6}$ For the moment, we continue to suppress the fixed $\tau=\tau_{\mathfrak{p}, i} \in \Sigma_{0}$ from the notation. 
$\mathcal{F}^{\bullet}$, and we thus obtain isomorphisms $\alpha_{\nu}: \nu^{*} \mathcal{P}_{\theta} \stackrel{\sim}{\longrightarrow} \mathcal{P}_{\theta}$ compatible with (5) and satisfying $\alpha_{\nu^{\prime} \nu}=\alpha_{\nu} \circ \nu^{*}\left(\alpha_{\nu^{\prime}}\right)$ (for $\left.\nu, \nu^{\prime} \in \mathcal{O}_{F,(p),+}^{\times}\right)$. Recall also that the action of $\mathcal{O}_{F,(p),+}^{\times}$on $\widetilde{Y}_{U}$ factors through $\mathcal{O}_{F,(p),+}^{\times} /\left(U \cap \mathcal{O}_{F}^{\times}\right)^{2}$, the isomorphism $\underline{A} \stackrel{\sim}{\longrightarrow} \nu^{*} \underline{A}$ being defined by $\iota\left(\mu^{-1}\right)$ if $\nu=\mu^{2}$ for $\mu \in U \cap \mathcal{O}_{F}^{\times}$, and one finds that the automorphism of $\mathcal{P}_{\theta}$ obtained from $\alpha_{\nu}$ is multiplication by $\theta(\mu)$, so the natural action of $\mathcal{O}_{F,(p),+}^{\times}$on the bundles fails to define descent data with respect to the cover $\widetilde{Y}_{U} \rightarrow Y_{U}$. We do, however, obtain descent data after taking suitable tensor products or base changes of these bundles, which we now consider.

For any $\mathcal{O}$-algebra $R$, we will use $\cdot_{R}$ to denote the base change to $R$ of an $\mathcal{O}$-scheme $X$, as well as the pullback to $X_{R}$ of a quasi-coherent sheaf on $X$. Let $\left\{\mathbf{e}_{\theta} \mid \theta \in \Sigma\right\}$ denote the standard basis of $\mathbb{Z}^{\Sigma}$. For $\mathbf{k}=\sum k_{\theta} \mathbf{e}_{\theta}$ and $\mathbf{l}=\sum l_{\theta} \mathbf{e}_{\theta} \in \mathbb{Z}^{\Sigma}$, we define the line bundle

$$
\widetilde{\mathcal{A}}_{\mathbf{k}, \mathbf{l}}=\bigotimes_{\theta \in \Sigma}\left(\mathcal{L}_{\theta}^{\otimes k_{\theta}} \otimes \mathcal{N}_{\theta}^{\otimes l_{\theta}}\right) \cong \bigotimes_{\theta \in \Sigma}\left(\mathcal{L}_{\theta}^{\otimes k_{\theta}+l_{\theta}} \otimes \mathcal{M}_{\theta}^{\otimes l_{\theta}}\right)
$$

on $S=\widetilde{Y}_{U}$, where all tensor products are over $\mathcal{O}_{S}$. For $\mathbf{n}=\sum n_{\theta} \mathbf{e}_{\theta} \in \mathbb{Z}^{\Sigma}$, we let $\chi_{\mathbf{n}}$ : $\mathcal{O}_{F}^{\times} \rightarrow \mathcal{O}^{\times}$denote the character defined by $\chi_{\mathbf{n}}(\mu)=\prod_{\theta} \theta(\mu)^{n_{\theta}}$, and we let $\chi_{\mathbf{n}, R}$ denote the associated $R^{\times}$-valued character. If $\mathbf{k}, \mathbf{l}, R$ and $U$ are such that $\chi_{\mathbf{k}+2 \mathbf{l}, R}$ is trivial on $\mathcal{O}_{F}^{\times} \cap U$, then the action of $\mathcal{O}_{F,(p),+}^{\times}$on $\widetilde{\mathcal{A}}_{\mathbf{k}, 1, R}$ (over its action on $\widetilde{Y}_{U, R}$ ) factors through $\mathcal{O}_{F,(p),+}^{\times} /\left(U \cap \mathcal{O}_{F}^{\times}\right)^{2}$ and hence defines descent data, in which case we denote the resulting line bundle on $Y_{U, R}$ by $\mathcal{A}_{\mathbf{k}, \mathbf{l}, R}$.

Definition 3.2.1. For $\mathbf{k}, \mathbf{l}, U$ and $R$ as above, we call $\mathcal{A}_{\mathbf{k}, 1, R}$ the automorphic line bundle of weight $(\mathbf{k}, \mathbf{l})$ on $Y_{U, R}$, and we define the space of Hilbert modular forms of weight $(\mathbf{k}, \mathbf{l})$ and level $U$ with coefficients in $R$ to be

$$
M_{\mathbf{k}, \mathbf{l}}(U ; R):=H^{0}\left(Y_{U, R}, \mathcal{A}_{\mathbf{k}, \mathbf{l}, R}\right) .
$$

We note some general situations in which this space is defined:

- The paritious setting: if $w=k_{\theta}+2 l_{\theta}$ is independent of $\theta$, then $\chi_{\mathbf{k}+2 \mathbf{l}}(\mu)=$ $\operatorname{Nm}_{F / \mathbb{Q}}(\mu)^{w}=1$ for all $\mu \in U \cap \mathcal{O}_{F}^{\times}$(assuming only $U$ is small enough that $U \cap \mathcal{O}_{F}^{\times}$ has no elements of norm -1 if $w$ is odd).

- The $\bmod p$ setting: if $R$ is any $\mathbb{F}=\mathcal{O} / \mathfrak{m}_{\mathcal{O}}$-algebra and $U$ is sufficiently small that $\mu \equiv 1 \bmod \mathfrak{p}$ for all $\mu \in U \cap \mathcal{O}_{F}^{\times}$and $\mathfrak{p} \in S_{p}$, then $\theta(\mu) \equiv 1 \bmod \mathfrak{m}_{\mathcal{O}}$ for all $\theta \in \Sigma$, so $\chi_{\mathbf{k}+2 \mathbf{l}, \mathbb{F}}$ is trivial on $U \cap \mathcal{O}_{F}^{\times}$, and hence so is $\chi_{\mathbf{k}+2 \mathbf{l}, R}$.

- The torsion setting: if $R$ is an $\mathcal{O} / p^{N} \mathcal{O}$-algebra and $U$ is sufficiently small that $\mu \equiv 1 \bmod p^{N} \mathcal{O}_{F}$ for all $\mu \in U \cap \mathcal{O}_{F}^{\times}$, then $\chi_{\mathbf{k}+2 \mathbf{l}, \mathcal{O} / p^{N} \mathcal{O}}$ is trivial on $U \cap \mathcal{O}_{F}^{\times}$, and hence so is $\chi_{\mathbf{k}+2 \mathbf{l}, R}$.

We also have a natural left action of $\mathrm{GL}_{2}\left(\mathbb{A}_{F, \mathbf{f}}^{(p)}\right)$ on the direct limit over $U$ of the spaces $M_{\mathbf{k}, \mathbf{l}}(U ; R)$. More precisely, suppose that $U_{1}$ and $U_{2}$ are as above and $g \in \mathrm{GL}_{2}\left(\mathbb{A}_{F, \mathbf{f}}^{(p)}\right)$ is such that $g^{-1} U_{1} g \subset U_{2}$, in which case recall that in Subsection 2.2 we defined a morphism $\widetilde{\rho}_{g}: \widetilde{Y}_{U_{1}} \rightarrow \widetilde{Y}_{U_{2}}$ descending to a morphism $\rho_{g}: Y_{U_{1}} \rightarrow Y_{U_{2}}$. Furthermore, the morphism $\widetilde{\rho}_{g}$ is obtained from a prime-to- $p$ quasi-isogeny $A \rightarrow A^{\prime}$ where $A$ is the universal abelian scheme over $\widetilde{Y}_{U_{1}}$ and $A^{\prime}$ is the pullback of the universal abelian scheme. We 
thus obtain isomorphisms $\widetilde{\rho}_{g}^{*} \mathcal{P}_{\theta, 2} \rightarrow \mathcal{P}_{\theta, 1}$ compatible with (5) and the action of $\mathcal{O}_{F,(p),+}^{\times}$ (augmenting the notation for the automorphic bundles on $\widetilde{Y}_{U_{i}}$ and $Y_{U_{i}, R}$ with the subscript $i$ ). Note that $U_{1} \cap \mathcal{O}_{F}^{\times} \subset U_{2} \cap \mathcal{O}_{F}^{\times}$, so if $\widetilde{\mathcal{A}}_{\mathbf{k}, \mathbf{l}, R, 2}$ descends to $Y_{U_{2}, R}$, then $\widetilde{\mathcal{A}}_{\mathbf{k}, \mathbf{l}, R, 1}$ descends to $Y_{U_{1}, R}$, and we obtain an isomorphism $\rho_{g}^{*} \mathcal{A}_{\mathbf{k}, \mathbf{l}, R, 2} \cong \mathcal{A}_{\mathbf{k}, \mathbf{l}, R, 1}$. We then define $[g]=[g]_{U_{1}, U_{2}}: M_{\mathbf{k}, \mathbf{l}}\left(U_{2} ; R\right) \rightarrow M_{\mathbf{k}, \mathbf{l}}\left(U_{1} ; R\right)$ as the composite

$$
H^{0}\left(Y_{U_{2}}, \mathcal{A}_{\mathbf{k}, \mathbf{l}, R, 2}\right) \stackrel{\rho_{g}^{*}}{\longrightarrow} H^{0}\left(Y_{U_{1}}, \rho_{g}^{*} \mathcal{A}_{\mathbf{k}, \mathbf{l}, R, 2}\right) \stackrel{\sim}{\longrightarrow} H^{0}\left(Y_{U_{1}}, \mathcal{A}_{\mathbf{k}, \mathbf{l}, R, 1}\right)
$$

These maps satisfy the obvious compatibility, namely, that

$$
\left[g_{1}\right]_{U_{1}, U_{2}} \circ\left[g_{2}\right]_{U_{2}, U_{3}}=\left[g_{1} g_{2}\right]_{U_{1}, U_{3}}
$$

whenever $g_{1}^{-1} U_{1} g_{1} \subset U_{2}$ and $g_{2}^{-1} U_{2} g_{2} \subset U_{3}$, and hence define an action of $\mathrm{GL}_{2}\left(\mathbb{A}_{F, \mathbf{f}}^{(p)}\right)$ on

$$
M_{\mathbf{k}, \mathbf{l}}(R):=\underset{U}{\lim } M_{\mathbf{k}, \mathbf{l}}(U ; R)
$$

(where the limit is over sufficiently small open compact $U$ with respect to the maps $\left.[1]_{U_{1}, U_{2}}\right)$. For paritious $\mathbf{k}, \mathbf{l}$ and any choice of $\mathcal{O} \rightarrow \mathbb{C}$, we may identify the spaces $M_{\mathbf{k}, \mathbf{l}}(U ; \mathbb{C})$ with those of holomorphic Hilbert modular forms, compatibly with the usual action (up to normalisation by a factor of $\|\operatorname{det} g\|$ depending on conventions).

Finally, we remark that the action of $\nu \in \mathcal{O}_{F,(p),+}^{\times}$on the trivialisation of $\mathcal{N}_{\theta}$ is given by multiplication by $\theta(\nu)$, so their products do not descend to trivialisations of line bundles on $Y_{U, R}$. However, since the stabiliser of each geometric connected component of $\widetilde{Y}_{U}$ is $\mathcal{O}_{F,+}^{\times} \cap \operatorname{det}(U)$, we can obtain a (noncanonical) trivialisation of $\mathcal{A}_{\mathbf{0}, \mathbf{l}, R}$ as in [12, Prop. 3.6.1], provided $\chi_{\mathbf{l}, R}$ is trivial on $\mathcal{O}_{F,+}^{\times} \cap \operatorname{det}(U)$ and the geometric connected components of $Y_{U}$ are defined over $R$. Furthermore, the same argument as in the proof of [12, Lemma 4.5.1] shows the following.

Proposition 3.2.2. If $p^{N} R=0$, then the action of $\mathrm{GL}_{2}\left(\mathbb{A}_{F, \mathbf{f}}^{(p)}\right)$ on $M_{\mathbf{0 , 1}}(R)$ factors through $\operatorname{det}: \mathrm{GL}_{2}\left(\mathbb{A}_{F, \mathbf{f}}^{(p)}\right) \rightarrow\left(\mathbb{A}_{F, \mathbf{f}}^{(p)}\right)^{\times} ;$Furthermore, as a representation of $\left(\mathbb{A}_{F, \mathbf{f}}^{(p)}\right)^{\times}, M_{\mathbf{0}, \mathbf{1}}(R)$ is isomorphic to the smooth induction of the character $\mathcal{O}_{F,(p),+}^{\times} \rightarrow R^{\times}$defined by $\alpha \mapsto$ $\prod_{\theta} \theta(\alpha)^{l_{\theta}}$.

\subsection{The Kodaira-Spencer filtration}

In this section we define a filtration on $\Omega_{Y_{U} / \mathcal{O}}^{1}$ whose pieces are described by automorphic bundles with weight components $k_{\theta}=2, l_{\theta}=-1$. The construction of the filtration is due to Reduzzi and Xiao (see $[32, \S 2.8]$ ), but their presentation is complicated by the fact that they wish to prove smoothness simultaneously, and it obscures the fact that the bundles we denoted $\mathcal{G}^{(j)}$ automatically satisfy the orthogonality condition appearing in the definition of their counterparts in [32]. We will show below that, with smoothness already established, one can give a more direct conceptual description of the filtration 
and its properties. ${ }^{7}$ Furthermore, in the case $p=2$, the argument in [32] appeals to a very general flatness assertion for divided power envelopes for which we could not find a proof or reference, so it is not used here.

Theorem 3.3.1 (Reduzzi-Xiao). There exists a decomposition

$$
\Omega_{Y_{U} / \mathcal{O}}^{1}=\bigoplus_{\mathfrak{p} \in S_{p}} \bigoplus_{i=1}^{f_{\mathfrak{p}}} \Omega_{Y_{U} / \mathcal{O}, \mathfrak{p}, i}^{1},
$$

together with an increasing filtration

$$
\begin{aligned}
& 0=\operatorname{Fil}^{0}\left(\Omega_{Y_{U} / \mathcal{O}, \mathfrak{p}, i}^{1}\right) \subset \operatorname{Fil}^{1}\left(\Omega_{Y_{U} / \mathcal{O}, \mathfrak{p}, i}^{1}\right) \quad \subset \cdots \\
& \subset \operatorname{Fil}^{e_{\mathfrak{p}}-1}\left(\Omega_{Y_{U} / \mathcal{O}, \mathfrak{p}, i}^{1}\right) \subset \operatorname{Fil}^{e_{\mathfrak{p}}}\left(\Omega_{Y_{U} / \mathcal{O}, \mathfrak{p}, i}^{1}\right)=\Omega_{Y_{U} / \mathcal{O}, \mathfrak{p}, i}^{1}
\end{aligned}
$$

for each $\mathfrak{p} \in S_{p}$ and $i=1, \ldots, f_{\mathfrak{p}}$, such that for each $j=1, \ldots, e_{\mathfrak{p}}, \operatorname{gr}^{j}\left(\Omega_{Y_{U} / \mathcal{O}, \mathfrak{p}, i}^{1}\right)$ is isomorphic to the automorphic bundle $\mathcal{A}_{2 \mathbf{e}_{\theta},-\mathbf{e}_{\theta}, \mathcal{O}}$, where $\theta=\theta_{\mathfrak{p}, i, j}$.

Proof. As usual, we first prove the analogous result for $S:=\widetilde{Y}_{U}$ and then descend to $Y_{U}$.

We let $\delta_{0}: S \stackrel{\sim}{\longrightarrow} \Delta \hookrightarrow Z_{0}$ denote the first infinitesimal thickening of the diagonal embedding, and we view $\Omega_{S / \mathcal{O}}^{1}$ as $\delta_{0}^{*} \mathcal{I}$, where $\mathcal{I}$ denotes the sheaf of ideals defining $\Delta$ in $Z_{0}$. Letting $s: A \rightarrow S$ denote the universal abelian scheme, the transition maps for the crystal $R^{1} s_{\text {cris }, *} \mathcal{O}_{A / \mathbb{Z}_{p}}$ and canonical isomorphisms with de Rham cohomology yield an $\mathcal{O}_{F} \otimes \mathcal{O}_{Z_{0}}$-linear isomorphism

$$
\alpha: p_{0}^{*} \mathcal{H}_{\mathrm{dR}}^{1}(A / S) \stackrel{\sim}{\longrightarrow} q_{0}^{*} \mathcal{H}_{\mathrm{dR}}^{1}(A / S)
$$

extending the identity on $S \cong \Delta$, where $p_{0}, q_{0}: Z_{0} \rightarrow S$ are the two projection maps $Z_{0} \rightarrow S$. Since $\alpha$ is $\mathcal{O}_{F}$-linear, it follows from the definition of $\mathcal{P}_{\tau, 1}=\mathcal{G}_{\tau}^{(1)}$ that $\alpha$ restricts to an isomorphism

$$
\alpha_{\tau, 1}: p_{0}^{*} \mathcal{P}_{\tau, 1} \stackrel{\sim}{\longrightarrow} q_{0}^{*} \mathcal{P}_{\tau, 1}
$$

for each $\mathfrak{p} \in S_{p}$ and $\tau \in \Sigma_{\mathfrak{p}, 0}$. Furthermore, since the composite

$$
p_{0}^{*} \mathcal{L}_{\tau, 1} \hookrightarrow p_{0}^{*} \mathcal{P}_{\tau, 1} \stackrel{\sim}{\longrightarrow} q_{0}^{*} \mathcal{P}_{\tau, 1} \rightarrow q_{0}^{*} \mathcal{M}_{\tau, 1}
$$

has trivial pullback to $S \cong \Delta$, it factors through a morphism

$$
\delta_{0, *} \mathcal{L}_{\tau, 1}=p_{0}^{*} \mathcal{L}_{\tau, 1} \otimes_{\mathcal{O}_{Z_{0}}}\left(\mathcal{O}_{Z_{0}} / \mathcal{I}\right) \longrightarrow q_{0}^{*} \mathcal{M}_{\tau, 1} \otimes_{\mathcal{O}_{Z_{0}}} \mathcal{I}=\delta_{0, *} \mathcal{M}_{\tau, 1} \otimes_{\mathcal{O}_{Z_{0}}} \mathcal{I}
$$

and hence induces a morphism

$$
\beta_{\tau, 1}: \delta_{0, *}\left(\mathcal{L}_{\tau, 1} \otimes_{\mathcal{O}_{S}} \mathcal{M}_{\tau, 1}^{-1}\right) \longrightarrow \mathcal{I}
$$

${ }^{7}$ The simultaneous treatment in [32] seems natural in view of the inherent overlap in the analysis of deformations needed for both results. However, the decision not to appeal to the results in [35] and [33] also makes reference to a perceived minor gap in the proof of [35, Prop 2.11]; we found no such gap, nor is that result even needed, but we remark that we made implicit use of [35, Cor. 2.10] when invoking the proof of [33, Prop. 6] to conclude that $\widetilde{Y}_{U}$ is smooth over $\mathcal{O}$. 
We then define the sheaf of ideals $\mathcal{I}_{\tau, 1}$ on $Z_{0}$ to be the image of $\beta_{\tau, 1}$, and we let $Z_{\tau, 1}$ denote the subscheme of $Z_{0}$ defined by $\mathcal{I}_{\tau, 1}$, and $p_{\tau, 1}$ and $q_{\tau, 1}$ the resulting projection maps $Z_{\tau, 1} \rightarrow S$. By construction, the pullback of $\beta_{\tau, 1}$ to $Z_{\tau, 1}$ is trivial, and hence so is that of the morphism $p_{0}^{*} \mathcal{L}_{\tau, 1} \rightarrow q_{0}^{*} \mathcal{M}_{\tau, 1}$, which implies that the pullback of $\alpha$ maps $p_{\tau, 1}^{*} \mathcal{L}_{\tau, 1}=p_{\tau, 1}^{*} \mathcal{F}_{\tau}^{(1)}$ isomorphically to $q_{\tau, 1}^{*} \mathcal{L}_{\tau, 1}=q_{\tau, 1}^{*} \mathcal{F}_{\tau}^{(1)}$. It follows from $\mathcal{O}_{F}$-linearity that $\alpha$ induces an isomorphism $p_{\tau, 1}^{*} \mathcal{G}_{\tau}^{(2)} \stackrel{\sim}{\longrightarrow} q_{\tau, 1}^{*} \mathcal{G}_{\tau}^{(2)}$ (if $e_{\mathfrak{p}}>1$ ), and hence an isomorphism

$$
\alpha_{\tau, 2}: p_{\tau, 1}^{*} \mathcal{P}_{\tau, 2} \stackrel{\sim}{\longrightarrow} q_{\tau, 1}^{*} \mathcal{P}_{\tau, 2} \text {. }
$$

The same argument as above now yields a morphism

$$
\beta_{\tau, 2}: \delta_{0, *}\left(\mathcal{L}_{\tau, 2} \otimes_{\mathcal{O}_{S}} \mathcal{M}_{\tau, 2}^{-1}\right) \longrightarrow \mathcal{I} / \mathcal{I}_{\tau, 1}
$$

whose image is that of a sheaf of ideals on $Z_{0}$ we denote by $\mathcal{I}_{\tau, 2}$.

Iterating the above construction thus yields, for each $\tau \in \Sigma_{\mathfrak{p}, 0}$, a chain of sheaves of ideals

$$
0=\mathcal{I}_{\tau, 0} \subset \mathcal{I}_{\tau, 1} \subset \cdots \subset \mathcal{I}_{\tau, e_{\mathfrak{p}}}
$$

on $Z_{0}$ such that $\alpha$ induces

- isomorphisms $p_{\tau, j}^{*} \mathcal{F}_{\tau}^{(j)} \stackrel{\sim}{\longrightarrow} q_{\tau, j}^{*} \mathcal{F}_{\tau}^{(j)}$

- and surjections $\delta_{0, *}\left(\mathcal{L}_{\tau, j} \otimes_{\mathcal{O}_{S}} \mathcal{M}_{\tau, j}^{-1}\right) \rightarrow \mathcal{I}_{\tau, j} / \mathcal{I}_{\tau, j-1}$

for $j=1, \ldots, e_{\mathfrak{p}}$, where $Z_{\tau, j}$ denotes the closed subscheme of $Z_{0}$ defined by $\mathcal{I}_{\tau, j}$ and $p_{\tau, j}$, $q_{\tau, j}$ are the projections $Z_{\tau, j} \rightarrow S$.

Furthermore, we claim that the map

$$
\bigoplus_{\mathfrak{p} \in S_{p}} \bigoplus_{\tau \in \Sigma_{\mathfrak{p}, 0}} \mathcal{I}_{\tau, e_{\mathfrak{p}}} \rightarrow \mathcal{I}
$$

is surjective. Indeed, let $\mathcal{J}$ denote the image and let $T$ denote the corresponding closed subscheme of $Z_{0}$, so $T$ is the scheme-theoretic intersection of the $Z_{\tau, e_{\mathfrak{p}}}$, and let ${ }^{8} p$, $q: T \rightarrow S$ denote the projection maps. By construction, $\alpha$ pulls back to an isomorphism $p^{*} \mathcal{H}_{\mathrm{dR}}^{1}(A / S) \stackrel{\sim}{\longrightarrow} q^{*} \mathcal{H}_{\mathrm{dR}}^{1}(A / S)$ under which $p^{*} \mathcal{F}_{\tau}^{(j)} \stackrel{\sim}{\longrightarrow} q^{*} \mathcal{F}_{\tau}^{(j)}$ for all $\tau$ and $j$. In particular, $t_{*} \Omega_{p^{*} A / T}^{1}=p^{*}\left(s_{*} \Omega_{A / S}^{1}\right) \stackrel{\sim}{\longrightarrow} q^{*}\left(s_{*} \Omega_{A / S}^{1}\right)=u_{*} \Omega_{q^{*} A / T}^{1}$ (where $t: p^{*} A \rightarrow T$ and $u: q^{*} A \rightarrow$ $T$ are the structure morphisms), which the Grothendieck-Messing theorem implies is induced by an isomorphism $p^{*} A \cong q^{*} A$ of abelian schemes lifting the identity over $S$. Since the isomorphism respects the filtrations $\mathcal{F}^{\bullet}$ and the lifts of the universal auxiliary structures $\iota, \lambda$ and $\eta$ over $T$ are unique, it follows that $p^{*} \underline{A} \cong q^{*} \underline{A}$, which means that $p=q \in S(T)$, so $T=\Delta$.

Now defining $\Omega_{S / \mathcal{O}, \mathfrak{p}, i}^{1}=\delta_{0}^{*} \mathcal{I}_{\tau_{\mathfrak{p}, i}, e_{\mathfrak{p}}}$ and $\mathrm{Fil}^{j}\left(\Omega_{S / \mathcal{O}, \mathfrak{p}, i}^{1}\right)=\delta_{0}^{*} \mathcal{I}_{\tau_{\mathfrak{p}, i}, j}$ for $\mathfrak{p} \in S_{p}, 1 \leq i \leq f_{\mathfrak{p}}$, $1 \leq j \leq e_{\mathfrak{p}}$, we obtain surjective morphisms

$$
\mathcal{L}_{\tau_{\mathfrak{p}, i}, j} \otimes_{\mathcal{O}_{S}} \mathcal{M}_{\tau_{\mathfrak{p}, i}, j}^{-1} \rightarrow \operatorname{gr}^{j}\left(\Omega_{S / \mathcal{O}, \mathfrak{p}, i}^{1}\right) \quad \text { and } \quad \bigoplus_{\mathfrak{p}, i} \Omega_{S / \mathcal{O}, \mathfrak{p}, i}^{1} \rightarrow \Omega_{S / \mathcal{O}}^{1}
$$

Since the $\mathcal{L}_{\tau_{\mathfrak{p}, i}, j} \otimes_{\mathcal{O}_{S}} \mathcal{M}_{\tau_{\mathfrak{p}, i}, j}^{-1}$ are line bundles and $\Omega_{S / \mathcal{O}}^{1}$ is locally free of rank $d$, it follows that all of the maps are isomorphisms.

${ }^{8}$ With apologies for the temporary dual use of $p$. 
Finally, the constructions above are independent of the polarisation $\lambda$ and hence are compatible with the action of $\nu \in \mathcal{O}_{F,(p),+}^{\times}$on $S=\widetilde{Y}_{U}$. More precisely, the pullback of $\alpha$ via the diagonal map $(\nu, \nu)$ is compatible with the canonical isomorphism $\nu^{*} \mathcal{H}_{\mathrm{dR}}^{1}(A / S) \cong$ $\mathcal{H}_{\mathrm{dR}}^{1}(A / S)$ induced by the identification of $\nu^{*} A$ with $A$, from which it follows easily that the morphisms in the construction of the filtration are invariant under the action of $\mathcal{O}_{F,(p),+}^{\times}$and hence descend to give the decomposition, filtrations and isomorphisms in the statement of the theorem.

Let us also note the interpretation of the Kodaira-Spencer filtration in terms of tangent spaces. For a closed point $y$ of $S$ corresponding to the data $\underline{A}_{0}=\left(A_{0}, \iota_{0}, \lambda_{0}, \eta_{0}, \mathcal{F}_{0}^{*}\right)$ over a finite extension $k$ of the residue field of $\mathcal{O}$, the fibre $T_{y}(S)$ of $\mathcal{H o m}_{\mathcal{O}_{S}}\left(\Omega_{S / \mathcal{O}}^{1}, \mathcal{O}_{S}\right)$ is canonically identified with the set of isomorphism classes of data $\underline{A}_{1}$ over $k[\epsilon]$ lifting $\underline{A}_{0}$, and the decomposition and filtrations of the theorem yield dual decompositions of $T_{y}(S)$ into components $T_{y}(S)_{\tau}$ with decreasing filtrations $\mathrm{Fil}^{j}\left(T_{y}(S)_{\tau}\right)$. From the proof of the theorem one sees immediately that $\bigoplus_{\tau} \mathrm{Fil}^{j_{\tau}}\left(T_{y}(S)_{\tau}\right)$ corresponds to the set of $\left(A_{1}, \iota_{1}, \lambda_{1}, \eta_{1}, \mathcal{F}_{1}^{\bullet}\right)$ such that $\mathcal{F}_{1, \tau}^{(j)}$ is the image of $\mathcal{F}_{0, \tau}^{(j)} \otimes_{k} k[\epsilon]$ for all $\tau$ and $j \leq j_{\tau}$ under the canonical isomorphism

$$
H_{\mathrm{dR}}^{1}\left(A_{1} / k[\epsilon]\right) \cong H_{\text {cris }}^{1}\left(A_{0} / k[\epsilon]\right) \cong H_{\mathrm{dR}}^{1}\left(A_{0} / k\right) \otimes_{k} k[\epsilon] .
$$

We note also that the theorem yields a canonical (Kodaira-Spencer) isomorphism

$$
\Omega_{Y_{U} / \mathcal{O}}^{d} \cong \wedge_{\mathcal{O}_{Y_{U}}}^{d} \Omega_{Y_{U} / \mathcal{O}}^{1} \cong \mathcal{A}_{2,-1, \mathcal{O}}
$$

(writing $\sum m \mathbf{e}_{\theta}$ as $\mathbf{m}$ for $m \in \mathbb{Z}$ ). Furthermore, the decomposition, filtrations and isomorphisms of the theorem (and hence also the Kodaira-Spencer isomorphism) are Hecke-equivariant in the obvious sense. More precisely, the same argument as for the compatibility with the $\mathcal{O}_{F,(p),+}^{\times}$-action, but using the quasi-isogeny in the construction of $\rho_{g}$, shows that if $U_{1}, U_{2}$ and $g \in \mathrm{GL}_{2}\left(\mathbb{A}_{F, \mathbf{f}}^{(p)}\right)$ are such that $g^{-1} U_{1} g \subset U_{2}$, then $\rho_{g}^{*} \operatorname{Fil}^{j}\left(\Omega_{Y_{U_{2}} / \mathcal{O}, \mathfrak{p}, i}^{1}\right)$ corresponds to $\operatorname{Fil}^{j}\left(\Omega_{Y_{U_{1}} / \mathcal{O}, \mathfrak{p}, i}^{1}\right)$ for all $\mathfrak{p}, i, j$ under the canonical isomorphism $\rho_{g}^{*} \Omega_{Y_{U_{2}} / \mathcal{O}}^{1} \stackrel{\sim}{\longrightarrow} \Omega_{Y_{U_{1}} / \mathcal{O}}^{1}$, and the resulting diagrams

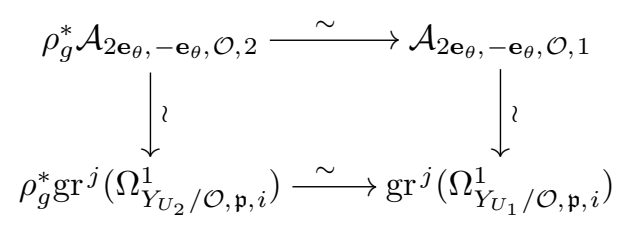

commute (where the top arrow is defined in the discussion preceding (6)).

\section{Partial Hasse invariants}

\subsection{Construction of $H_{\theta}$ and $G_{\theta}$}

We now recall the definition, due to Reduzzi and Xiao [32], of generalised partial Hasse invariants on Pappas-Rapoport models. These will be, for each $\theta=\theta_{\mathfrak{p}, i, j} \in \Sigma$, a Hilbert modular form $H_{\theta}$ of weight $\left(\mathbf{h}_{\theta}, \mathbf{0}\right)$ with coefficients in $\mathbb{F}=\mathcal{O} / \mathfrak{m}_{\mathcal{O}}$, where 
$\mathbf{h}_{\theta}:=n_{\theta} \mathbf{e}_{\sigma^{-1} \theta}-\mathbf{e}_{\theta}$, with $n_{\theta}=p$ if $j=1$ and $n_{\theta}=1$ if $j>1$. We also define below a(n in)variant $G_{\theta}$ of weight $\left(\mathbf{0}, \mathbf{h}_{\theta}\right)$.

We will now be working in the $\bmod p$ setting, so until further notice $S$ will denote $\widetilde{Y}_{U, \mathbb{F}}$, and $s: A \rightarrow S$ the universal abelian scheme over it. Thus, $\mathcal{H}_{\mathrm{dR}}^{1}(A / S)$ is a locally free sheaf of rank 2 over

$$
\mathcal{O}_{F} \otimes \mathcal{O}_{S} \cong \bigoplus_{\mathfrak{p} \in S_{p}} \bigoplus_{\tau \in \Sigma_{\mathfrak{p}, 0}} \mathcal{O}_{S}[u] /\left(u^{e_{\mathfrak{p}}}\right),
$$

where $u$ acts via $\iota\left(\varpi_{\mathfrak{p}}\right)^{*}$ on the $\mathfrak{p}$-component of

$$
\mathcal{H}_{\mathrm{dR}}^{1}(A / S)=\bigoplus_{\mathfrak{p} \in S_{p}} \mathcal{H}_{\mathfrak{p}}=\bigoplus_{\mathfrak{p} \in S_{p}} \bigoplus_{\tau \in \Sigma_{\mathfrak{p}, 0}} \mathcal{H}_{\tau}
$$

We will also now be working with a fixed $\mathfrak{p}$ and omit the subscript from the notation, so that

$$
\mathcal{H}=\mathcal{H}_{\mathrm{dR}}^{1}(A / S)_{\mathfrak{p}}=\bigoplus_{\tau \in \Sigma_{\mathfrak{p}, 0}} \mathcal{H}_{\tau}=\bigoplus_{i \in \mathbb{Z} / f \mathbb{Z}} \mathcal{H}_{i}
$$

with each $\mathcal{H}_{i}$ locally free of rank 2 over $\mathcal{O}_{S}[u] /\left(u^{e}\right)$ (where we have also abbreviated the subscript $\tau_{\mathfrak{p}, i}$ by $i$ ). Furthermore, for each $i \in \mathbb{Z} / f \mathbb{Z}$, we have a filtration

$$
0=\mathcal{F}_{i}^{(0)} \subset \mathcal{F}_{i}^{(1)} \subset \cdots \subset \mathcal{F}_{i}^{(e-1)} \subset \mathcal{F}_{i}^{(e)}=\left(s_{*} \Omega_{A / S}^{1}\right)_{i}
$$

by sheaves of $\mathcal{O}_{S}[u] /\left(u^{e}\right)$-modules such that the quotients $\mathcal{L}_{i, j}=\mathcal{F}_{i}^{(j)} / \mathcal{F}_{i}^{(j-1)}$ are line bundles annihilated by $u$.

Firstly, note that if $j>1$, then $u: \mathcal{F}_{i}^{(j)} \rightarrow \mathcal{F}_{i}^{(j-1)}$ induces a morphism $\mathcal{L}_{i, j} \rightarrow \mathcal{L}_{i, j-1}$. On the other hand, if $j=1$, then the Verschiebung morphism $\phi_{S}^{*} A \rightarrow A$ over $S$ induces $\mathcal{O}_{S}[u] /\left(u^{e}\right)$-linear morphisms

$$
\operatorname{Ver}_{i}^{*}: \mathcal{H}_{i}=\mathcal{H}_{\tau_{i}} \longrightarrow \mathcal{H}_{\mathrm{dR}}^{1}\left(\phi_{S}^{*}(A) / S\right)_{\tau_{i}} \cong \phi_{S}^{*}\left(\mathcal{H}_{\tau_{i-1}}\right)=\phi_{S}^{*}\left(\mathcal{H}_{i-1}\right)
$$

with image $\phi_{S}^{*}\left(\mathcal{F}_{i-1}^{(e)}\right)$ (where $\phi_{S}$ denotes the absolute Frobenius on $S$ ). Note that $\mathcal{L}_{i, 1}=$ $\mathcal{F}_{i}^{(1)} \subset u^{e-1} \mathcal{H}_{i}$, so that $u^{e-1}$ defines an isomorphism

$$
u^{1-e} \mathcal{L}_{i, 1} / u \mathcal{H}_{i} \stackrel{\sim}{\longrightarrow} \mathcal{F}_{i}^{(1)}
$$

and that $\operatorname{Ver}_{i}^{*}\left(u \mathcal{H}_{i}\right)=u \phi_{S}^{*}\left(\mathcal{F}_{i-1}^{(e)}\right) \subset \phi_{S}^{*}\left(\mathcal{F}_{i-1}^{(e-1)}\right)$, so we obtain a well-defined $\mathcal{O}_{S}$-linear morphism ' $\operatorname{Ver}_{i}^{*} \circ u^{1-e}$,

$$
\mathcal{L}_{i, 1} \stackrel{\sim}{\longleftarrow} u^{1-e} \mathcal{L}_{i, 1} / u \mathcal{H}_{i} \longrightarrow \phi_{S}^{*}\left(\mathcal{L}_{i-1, e}\right) \cong \mathcal{L}_{i-1, e}^{\otimes p}
$$

We have now defined a morphism $\mathcal{L}_{\theta} \longrightarrow \mathcal{L}_{\sigma^{-1} \theta}^{\otimes n_{\theta}}$ for all $\theta$ and hence a section of $\widetilde{\mathcal{A}}_{\mathbf{h}_{\theta}, \mathbf{0}, \mathbb{F}}$ over $S$. Furthermore, it is straightforward to check that the section is invariant under the action of $\mathcal{O}_{F,(p),+}^{\times}$and therefore descends to an element

$$
H_{\theta} \in M_{\mathbf{h}_{\theta}, \mathbf{0}}(U ; \mathbb{F})=H^{0}\left(Y_{U, \mathbb{F}}, \mathcal{A}_{\mathbf{h}_{\theta}, \mathbf{0}, \mathbb{F}}\right)
$$


which we call the partial Hasse invariant (indexed by $\theta$ ). Furthermore, the partial Hasse invariants are stable under the Hecke action, in the sense that if $U_{1}, U_{2}$ and $g \in \mathrm{GL}_{2}\left(\mathbb{A}_{F, \mathbf{f}}^{(p)}\right)$ are such that $g^{-1} U_{1} g \subset U_{2}$, then $[g] H_{\theta, 2}=H_{\theta, 1}$. (Note also that the partial Hasse invariants are dependent on the choice of uniformiser $\varpi=\varpi_{\mathfrak{p}}$ only up to a scalar in $\mathbb{F}^{\times}:$if $\varpi$ is replaced by $a \varpi$ for some $a \in \mathcal{O}_{F, \mathfrak{p}}^{\times}$, then $H_{\theta}$ is replaced by $\tau(a) H_{\theta}$ if $j>1$ and by $\tau(a)^{1-e} H_{\theta}$ if $j=1$.)

We remark also that the line bundles $\mathcal{A}_{\mathbf{0}, \mathbf{h}_{\theta}, \mathbb{F}}$ have canonical trivialisations. Indeed, for each $i \in \mathbb{Z} / f \mathbb{Z}$ and $j=2, \ldots, e$, we have the exact sequence

$$
0 \rightarrow \mathcal{G}_{i}^{(j-1)} / \mathcal{F}_{i}^{(j-1)} \longrightarrow \mathcal{G}_{i}^{(j)} / \mathcal{F}_{i}^{(j-1)} \stackrel{u}{\longrightarrow} \mathcal{F}_{i}^{(j-1)} / \mathcal{F}_{i}^{(j-2)} \rightarrow 0
$$

over $S$; that is, $0 \rightarrow \mathcal{M}_{i, j-1} \rightarrow \mathcal{P}_{i, j} \rightarrow \mathcal{N}_{i, j-1} \rightarrow 0$, inducing an isomorphism

$$
\mathcal{N}_{i, j-1}=\mathcal{L}_{i, j-1} \otimes_{\mathcal{O}_{S}} \mathcal{M}_{i, j-1} \cong \wedge_{\mathcal{O}_{S}}^{2} \mathcal{P}_{i, j}=\mathcal{N}_{i, j}
$$

and hence $\mathcal{O}_{S} \simeq \mathcal{N}_{i, j-1}^{-1} \otimes_{\mathcal{O}_{S}} \mathcal{N}_{i, j}=\widetilde{\mathcal{A}}_{\mathbf{0}, \mathbf{h}_{\theta}, \mathbb{F}}$ for $\theta=\theta_{\mathfrak{p}, i, j}$, which it is straightforward to check descends to $Y_{U, \mathbb{F}}$. Similarly, we have the exact sequence

$$
0 \rightarrow \phi_{S}^{*}\left(\mathcal{G}_{i-1}^{(e)} / \mathcal{F}_{i-1}^{(e)}\right) \stackrel{\operatorname{Frob}_{A}^{*}}{\longrightarrow} \mathcal{G}_{i}^{(1)} \stackrel{\operatorname{Ver}_{A}^{*} u^{1-e}}{\longrightarrow} \phi_{S}^{*}\left(\mathcal{F}_{i-1}^{(e)} / \mathcal{F}_{i-1}^{(e-1)}\right) \rightarrow 0
$$

inducing an isomorphism $\phi_{S}^{*}\left(\mathcal{N}_{i-1, e}\right) \cong \mathcal{N}_{i, 1}$ and hence $\mathcal{O}_{S} \simeq \widetilde{\mathcal{A}}_{\mathbf{0}, \mathbf{h}_{\theta}, \mathbb{F}}$ for $\theta=\theta_{\mathfrak{p}, i, 1}$ descending to $Y_{U, \mathbb{F}}$. Furthermore, these isomorphisms are Hecke-equivariant in the usual sense, but note that they depend via $u$ on the choice of $\varpi_{\mathfrak{p}}$. For each $\theta$, we let $G_{\theta} \in M_{\mathbf{0}, \mathbf{h}_{\theta}}(U ; \mathbb{F})$ denote the canonical trivialising section.

\subsection{Stratification}

We also recall how the partial Hasse invariants define a stratification of the Hilbert modular variety in characteristic $p$. For any $\theta \in \Theta$, we define $\widetilde{Z}_{\theta}$ (respectively $Z_{\theta}$ ) to be the closed subscheme of $S=\widetilde{Y}_{U, \mathbb{F}}$ (respectively $Y_{U, \mathbb{F}}$ ) defined by the vanishing of $H_{\theta}$, and for any subset $T \subset \Sigma$, we let

$$
\widetilde{Z}_{T}=\bigcap_{\theta \in T} \widetilde{Z}_{\theta} \quad \text { and } \quad Z_{T}=\bigcap_{\theta \in T} Z_{\theta} .
$$

Note that the schemes $Z_{T}$ are stable under the Hecke action, in the strong sense that $Z_{T, 1}$ is the pullback of $Z_{T, 2}$ under $\rho_{g}: Y_{U_{1}} \rightarrow Y_{U_{2}}$.

We then have the following consequence ([32, Thm. 3.10]) of the description of the Kodaira-Spencer filtration on tangent spaces at closed points; we give a proof here as some of the details are relevant to the construction of $\Theta$-operators in Subsection 5.2.

Proposition 4.2.1. The schemes $\widetilde{Z}_{T}$ and $Z_{T}$ are smooth over $\mathbb{F}$ of dimension $|\Sigma-T|$.

Proof. We prove the result for $\widetilde{Z}_{T}$, from which the result for $Z_{T}$ is immediate.

Let $y$ be a closed point of $S$ with local ring $R=\mathcal{O}_{S, y}$, maximal ideal $\mathfrak{m}$ and residue field $k=R / \mathfrak{m}$. For each $\theta \in \Sigma$, choose a basis $b_{\theta}$ for $\mathcal{L}_{\theta, y}$ over $R$ and write $H_{\theta, y} b_{\theta}=x_{\theta} b_{\sigma^{-1} \theta_{\theta}}^{n_{\theta}}$. Thus, if $y \in \widetilde{Z}_{\theta}$, then $x_{\theta} \in \mathfrak{m}$, and we let $\bar{x}_{\theta}$ denote its image in $\mathfrak{m} / \mathfrak{m}^{2}$. 
Identifying $\mathfrak{m} / \mathfrak{m}^{2}$ with the fibre of $\Omega_{\widetilde{Y}_{U} / \mathcal{O}}^{1}$ at $y$ and writing $\mathrm{Fil}^{j}\left(\mathfrak{m} / \mathfrak{m}^{2}\right)_{\tau}$ for the subspaces obtained from the Kodaira-Spencer filtration, we claim that if $y \in Z_{\theta}$, then

$$
\operatorname{Fil}^{j}\left(\mathfrak{m} / \mathfrak{m}^{2}\right)_{\tau}=k \bar{x}_{\theta}+\operatorname{Fil}^{j-1}\left(\mathfrak{m} / \mathfrak{m}^{2}\right)_{\tau},
$$

where $\tau=\tau_{\mathfrak{p}, i}$ and $\theta=\theta_{\mathfrak{p}, i, j}$. Comparing dimensions, we see it suffices to prove the inclusion of the left-hand side in the right or, equivalently, that if

$$
v \in T_{y}(S)=\bigoplus_{\tau^{\prime} \in \Sigma_{0}} T_{y}(S)_{\tau^{\prime}}
$$

is such that its $\tau$-component $v_{\tau}$ lies in $\mathrm{Fil}^{j-1} T_{y}(S)_{\tau}$ and $v$ is orthogonal to $\bar{x}_{\theta}$, then in fact $v_{\tau} \in \mathrm{Fil}^{j}\left(T_{y}(S)_{\tau}\right)$ (using the notation of the discussion following the proof of Theorem 3.3.1).

Let $\underline{A}_{0}=\left(A_{0}, \iota_{0}, \lambda_{0}, \eta_{0}, \mathcal{F}_{0}^{\bullet}\right)$ denote the data corresponding to the point $y \in S(k)$ and $\underline{A}_{1}=\left(A_{1}, \iota_{1}, \lambda_{1}, \eta_{1}, \mathcal{F}_{1}^{\bullet}\right)$ that of its lift $v \in S(k[\epsilon])$. With $\tau=\tau_{\mathfrak{p}, i}$ fixed for now, we will suppress $\mathfrak{p}$ from the notation and replace the subscript $\tau_{p, i^{\prime}}$ by $i^{\prime}$ (for $i^{\prime}=i, i-1$ ). Recall the assumption that $v_{i} \in \mathrm{Fil}^{j-1}\left(T_{y}(S)_{i}\right)$ means that $\mathcal{F}_{1, i}^{\left(j^{\prime}\right)}$ corresponds to $\mathcal{F}_{0, i}^{\left(j^{\prime}\right)} \otimes_{k} k[\epsilon]$ for $j^{\prime}=1, \ldots, j-1$ under the canonical isomorphism

$$
H_{\mathrm{dR}}^{1}\left(A_{1} / k[\epsilon]\right) \cong H_{\mathrm{cris}}^{1}\left(A_{0} / k[\epsilon]\right) \cong H_{\mathrm{dR}}^{1}\left(A_{0} / k\right) \otimes_{k} k[\epsilon] .
$$

For $v_{i}$ to be orthogonal to $\bar{x}_{\theta}$ means that the morphism

$$
\mathcal{L}_{1, \theta} \longrightarrow \mathcal{L}_{1, \sigma^{-1} \theta}^{\otimes n_{\theta}}
$$

induced by $H_{\theta}$ vanishes, and we need to show this implies that $\mathcal{F}_{1, i}^{(j)}$ is the image of $\mathcal{F}_{0, i}^{(j)} \otimes_{k} k[\epsilon]$.

Suppose first that $j>1$. Then (9) is simply

$$
u: \mathcal{F}_{1, i}^{(j)} / \mathcal{F}_{1, i}^{(j-1)} \longrightarrow \mathcal{F}_{1, i}^{(j-1)} / \mathcal{F}_{1, i}^{(j-2)},
$$

whose vanishing means $\mathcal{F}_{1, i}^{(j)}=u^{-1} \mathcal{F}_{1, i}^{(j-2)}$. Since (8) sends $\mathcal{F}_{1, i}^{(j-2)}$ to $\mathcal{F}_{0, i}^{(j-2)} \otimes_{k} k[\epsilon]$ and is compatible with $u$, it follows that it also sends $\mathcal{F}_{1, i}^{(j)}$ to $\mathcal{F}_{0, i}^{(j)} \otimes_{k} k[\epsilon]$.

On the other hand, if $j=1$, then the vanishing of (9) means that $u^{1-e} \mathcal{F}_{1, i}^{(1)}$ is the preimage of $\phi_{1}^{*}\left(\mathcal{F}_{1, i-1}^{(e-1)}\right)$ under $\operatorname{Ver}_{i}^{*}$ (where $\phi_{1}$ is the absolute Frobenius on $k[\epsilon]$, and $\phi_{0}$ will denote the absolute Frobenius on $k$ ). Since the diagram

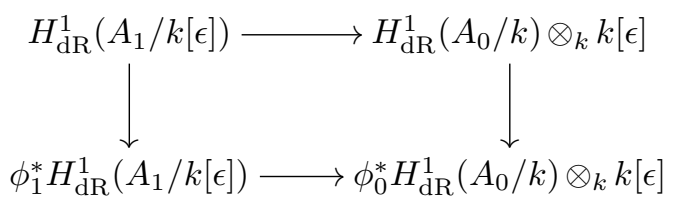

commutes, where the vertical maps are induced by Verschiebung, the top arrow is (8) and the bottom one is given by the identification of $\phi_{1}^{*} A_{1}$ with $\phi_{0}^{*} A_{0} \otimes_{k} k[\epsilon]$, so in particular identifies $\phi_{1}^{*}\left(\mathcal{F}_{1, i-1}^{(e-1)}\right)$ with $\phi_{0}^{*}\left(\mathcal{F}_{0, i-1}^{(e-1)}\right) \otimes_{k} k[\epsilon]$, it follows that the top arrow sends $u^{1-e} \mathcal{F}_{1, i}^{(1)}$ to $u^{1-e} \mathcal{F}_{0, i}^{(1)} \otimes_{k} k[\epsilon]$, and hence $\mathcal{F}_{1, i}^{(1)}$ to $\mathcal{F}_{0, i}^{(1)} \otimes_{k} k[\epsilon]$. This completes the proof of the claim. 
Now note that if $y \in \widetilde{Z}_{T}$, then (7) implies that the elements $\bar{x}_{\theta}$ for $\theta \in T$ can be extended to a basis for $\mathfrak{m} / \mathfrak{m}^{2}$ over $k$ and hence are linearly independent. Since $R$ is regular of dimension $d=|\Sigma|$, it follows that $\mathcal{O}_{\widetilde{Z}_{T}, y}=R /\left\langle x_{\theta}\right\rangle_{\theta \in T}$ is regular of dimension of $d-|T|$ and hence that $\widetilde{Z}_{T}$ is smooth over $\mathbb{F}$ of dimension $d-|T|$.

Finally, we recall the definition of the minimal weight of a nonzero mod $p$ Hilbert modular form. If $f \in M_{\mathbf{k}, \mathbf{l}}(U ; \mathbb{F})$, then $\mathbf{k}_{\min }(f)$ is defined to be $\mathbf{k}-\sum_{\theta} m_{\theta} \mathbf{h}_{\theta}$ where $\sum_{\theta} m_{\theta} \mathbf{e}_{\theta}$ is the unique maximal element of the set

$$
\left\{\sum_{\theta} m_{\theta} \mathbf{e}_{\theta} \in \mathbb{Z}_{\geq 0}^{\Sigma} \mid f=g \prod_{\theta \in \Sigma} H_{\theta}^{m_{\theta}} \text { for some } g \in M_{\mathbf{k}-\sum_{\theta} m_{\theta} \mathbf{h}_{\theta}, \mathbf{l}}(U ; \mathbb{F})\right\} .
$$

By the main result of [10], the minimal weight of $f$ always lies in the cone

$$
\Xi^{\min }:=\left\{\sum_{\theta} k_{\theta} \mathbf{e}_{\theta} \mid n_{\theta} k_{\theta} \geq k_{\sigma^{-1} \theta} \text { for all } \theta \in \Sigma\right\} \text {. }
$$

Note that the result stated in [10] applies to forms on a finite étale cover of $Y_{U, \mathbb{F}}$, from which the analogous result for forms on $Y_{U, \mathbb{F}}$ is immediate.

\section{Partial Theta operators}

\subsection{Fundamental Hasse invariants}

In order to define the partial $\Theta$-operators (in Subsection 5.2), we first define a canonical factorisation of the partial Hasse invariants over a finite flat (Igusa) cover of the Hilbert modular variety over $\mathbb{F}$.

We fix a sufficiently small $U$ that the line bundles $\mathcal{L}_{\theta}, \mathcal{M}_{\theta}, \mathcal{N}_{\theta}$ (and hence $\widetilde{\mathcal{A}}_{\mathbf{k}, \mathbf{l}, \mathbb{F}}$ ) on $\widetilde{Y}_{U, \mathbb{F}}$ descend to $Y_{U, \mathbb{F}}$ for all $\theta \in \Sigma$ (and all $\mathbf{k}, \mathbf{l} \in \mathbb{Z}^{\Sigma}$ ), and we write simply $\bar{Y}$ for $Y_{U, \mathbb{F}}$ and $\overline{\mathcal{L}}_{\tau, j}, \overline{\mathcal{M}}_{\tau, j}$ and $\overline{\mathcal{N}}_{\tau, j}$ for the line bundles on $\bar{Y}$. For each $\mathfrak{p} \in S_{p}$ and $\tau \in \Sigma_{\mathfrak{p}, 0}$, we let

$$
H_{\tau}=\prod_{j=1}^{e_{\mathfrak{p}}} H_{\tau, j} \in H^{0}\left(\bar{Y}, \overline{\mathcal{L}}_{\tau, e_{\mathfrak{p}}}^{-1} \otimes_{\mathcal{O}_{\bar{Y}}} \overline{\mathcal{L}}_{\phi^{-1} \circ \tau, e_{\mathfrak{p}}}^{\otimes p}\right)
$$

Viewing each $H_{\tau}$ as a morphism $\left(\overline{\mathcal{L}}_{\phi^{-1} \circ \tau, e_{\mathfrak{p}}}^{-1}\right)^{\otimes p} \rightarrow \overline{\mathcal{L}}_{\tau, e_{\mathfrak{p}}}^{-1}$ and $H_{\mathfrak{p}}:=\prod_{\tau \in \Sigma_{\mathfrak{p}, 0}} H_{\tau}$ as a morphism $\otimes_{\tau \in \Sigma_{\mathfrak{p}, 0}}\left(\overline{\mathcal{L}}_{\tau, e_{\mathfrak{p}}}^{-1}\right)^{\otimes(p-1)} \rightarrow \mathcal{O}_{\bar{Y}}$, we define the Igusa cover ${ }^{9}$ of $\bar{Y}$ (of level $\left.\mathfrak{q}=\prod \mathfrak{p}\right)$ to be

$$
Y^{\mathrm{Ig}}=\operatorname{Spec}\left(\operatorname{Sym}_{\mathcal{O}_{\bar{Y}}}\left(\bigoplus_{\tau \in \Sigma_{0}} \overline{\mathcal{L}}_{\tau, e_{\mathfrak{p}}}^{-1}\right) / \mathcal{I}\right)
$$

where $\mathcal{I}$ is the sheaf of ideals generated by the $\mathcal{O}_{\bar{Y}}$-submodules

$$
\left(H_{\tau}-1\right) \overline{\mathcal{L}}_{\tau, e_{\mathfrak{p}}}^{-1} \text { for } \tau \in \Sigma_{0}, \text { and }\left(H_{\mathfrak{p}}-1\right)\left(\bigotimes_{\tau \in \Sigma_{\mathfrak{p}, 0}}\left(\overline{\mathcal{L}}_{\tau, e_{\mathfrak{p}}}^{-1}\right)^{\otimes(p-1)}\right) \text { for } \mathfrak{p} \in S_{p}
$$

${ }^{9}$ The cover has a natural moduli-theoretic interpretation in terms of $A[\mathfrak{q}]$, but we will not need this here. 
(where all tensor products are over $\left.\mathcal{O}_{\bar{Y}}\right)$. We then define an action of $\left(\mathcal{O}_{F} / \mathfrak{q}\right)^{\times}$on $Y^{\text {Ig }}$ over $\bar{Y}$ by letting $\alpha \in\left(\mathcal{O}_{F} / \mathfrak{q}\right)^{\times}$act on the structure sheaf by the automorphism of sheaves of $\mathcal{O}_{\bar{Y}}$-algebras induced by multiplication by $\tau(\alpha)^{-1}$ on $\overline{\mathcal{L}}_{\tau, e_{\mathfrak{p}}}^{-1}$ for each $\tau$. We then see, exactly as in the proof of parts (1) and (2) of [12, Prop. 8.1.1], that the projection $\pi: Y^{\mathrm{Ig}} \rightarrow \bar{Y}$ is finite flat, generically étale and identifies $\bar{Y}$ with the quotient of $Y^{\mathrm{Ig}}$ by the action of $\left(\mathcal{O}_{F} / \mathfrak{q}\right)^{\times}$.

For each $\tau \in \Sigma_{\mathfrak{p}, 0}$, we let $h_{\tau, e_{\mathfrak{p}}}$ denote the tautological section of $\pi^{*} \overline{\mathcal{L}}_{\tau, e_{\mathfrak{p}}}$ induced by the inclusion $\overline{\mathcal{L}}_{\tau, e_{\mathfrak{p}}}^{-1} \hookrightarrow \pi_{*} \mathcal{O}_{Y^{\mathrm{Ig}}}$. We also define the section

$$
h_{\tau, j}=\pi^{*}\left(H_{\tau, j+1} \cdots H_{\tau, e_{\mathfrak{p}}}\right) h_{\tau, e_{\mathfrak{p}}}
$$

of $\pi^{*} \overline{\mathcal{L}}_{\tau, j}$ for $j=1, \ldots, e_{\mathfrak{p}}-1$. Note that since $Y^{\text {Ig }}$ is reduced (or since $\prod_{\tau \in \Sigma_{\mathfrak{p}, 0}} h_{\tau, e_{\mathfrak{p}}}^{p-1}=\pi^{*} H_{\mathfrak{p}}$ by construction), the sections $h_{\tau, e_{\mathfrak{p}}}$ are injective, and hence so are the $h_{\tau, j}$ for all $\tau$ and $j$. We write simply $h_{\theta}$ for the section $h_{\tau, j}$ of $\pi^{*} \overline{\mathcal{L}}_{\theta}=\pi^{*} \overline{\mathcal{L}}_{\tau, j}$, and we call the $h_{\theta}$ the fundamental Hasse invariant (indexed by $\theta$ ).

\subsection{Construction of $\Theta_{\tau}$}

We now explain how the construction of $\Theta$-operators in [12] directly generalises to the case where $p$ is ramified in $F$, yielding an operator that shifts the weight $\mathbf{k}$ by $(1,1)$ in the final two components corresponding to embeddings with the same reduction; that is, $\theta_{\mathfrak{p}, i, e_{\mathfrak{p}}-1}, \theta_{\mathfrak{p}, i, e_{\mathfrak{p}}}$ (and hence, by composing with multiplication by partial Hasse invariants, one can shift weights by +1 for any pair of embeddings with the same reduction).

Indeed, for each $\tau \in \Sigma_{0}$, we define the operator $\Theta_{\tau}$ exactly as in $[12, \S 8]$ but using the morphism

$$
K S_{\tau}: \Omega_{\bar{Y} / \mathbb{F}}^{1} \longrightarrow \operatorname{gr}^{e_{\mathfrak{p}}}\left(\Omega_{\bar{Y} / \mathbb{F}}^{1}\right)_{\tau} \stackrel{\sim}{\longrightarrow} \overline{\mathcal{L}}_{\tau, e_{\mathfrak{p}}} \otimes_{\mathcal{O}_{\bar{Y}}} \overline{\mathcal{M}}_{\tau, e_{\mathfrak{p}}}^{-1}
$$

provided by Theorem 3.3.1 via projection to the top graded piece of the filtration of the $\tau$-component of $\Omega_{Y / \mathbb{F}}^{1}$. More precisely, fix $\mathfrak{p}_{0} \in S_{p}$ and $\tau_{0}=\tau_{\mathfrak{p}_{0}, i}$, let $\theta_{0}=\theta_{\mathfrak{p}_{0}, i, e_{\mathfrak{p}_{0}}}$ and consider the morphism

$$
K S_{\tau_{0}}^{\mathrm{Ig}}: \Omega_{Y^{\mathrm{Ig}} / \mathbb{F}}^{1} \otimes_{\mathcal{O}_{Y^{\mathrm{Ig}}}} \mathcal{F}^{\mathrm{Ig}} \cong \pi^{*} \Omega_{\bar{Y} / \mathbb{F}}^{1} \otimes_{\mathcal{O}_{Y^{\mathrm{Ig}}}} \mathcal{F}^{\mathrm{Ig}} \longrightarrow \pi^{*}\left(\mathcal{A}_{2 \mathbf{e}_{\theta_{0}},-\mathbf{e}_{\theta_{0}}, \mathbb{F}}\right) \otimes_{\mathcal{O}_{Y^{I g}}} \mathcal{F}^{\mathrm{Ig}}
$$

induced by $K S_{\tau_{0}}$, where $\mathcal{F}^{\mathrm{Ig}}$ is the sheaf of total fractions on $Y^{\mathrm{Ig}}$. Suppose now that $f \in M_{\mathbf{k}, \mathbf{l}}(U ; \mathbb{F})$, and write $h^{\mathbf{k}}=\prod_{\theta \in \Sigma} h_{\theta}^{k_{\theta}}$ and $g^{\mathbf{l}}=\prod_{\theta \in \Sigma} g_{\theta}^{l_{\theta}}$ for any choice of trivialisations $g_{\theta}$ of the line bundles $\overline{\mathcal{N}}_{\theta}$ on $\bar{Y}$. We then define the section

$$
\Theta_{\tau_{0}}^{\mathrm{Ig}}(f):=h^{\mathbf{k}} \pi^{*}\left(g^{\mathbf{l}} H_{\theta_{0}}\right) K S_{\tau_{0}}^{\mathrm{Ig}}\left(d\left(h^{-\mathbf{k}} \pi^{*}\left(g^{-\mathbf{l}} f\right)\right)\right),
$$

where

$$
\mathbf{k}^{\prime}=\mathbf{k}+n_{\theta_{0}} \mathbf{e}_{\sigma^{-1} \theta_{0}}+\mathbf{e}_{\theta_{0}} \quad \text { and } \quad \mathbf{l}^{\prime}=\mathbf{l}+\mathbf{e}_{\theta_{0}} .
$$

Furthermore, the section is independent of the choices of $g_{\theta}$ and invariant under the action of $\left(\mathcal{O}_{F} / \mathfrak{q}\right)^{\times}$and hence descends to a section of $\mathcal{A}_{\mathbf{k}^{\prime}, \mathbf{l}^{\prime}, \mathbb{F}} \otimes_{\mathcal{O}_{\bar{Y}}} \mathcal{F}$, where $\mathcal{F}$ is the sheaf of total fractions on $\bar{Y}$. Denoting the section $\Theta_{\tau_{0}}(f)$, we have the following generalisation of [12, Thm. 8.2.2]. 
Theorem 5.2.1. If $f \in M_{\mathbf{k}, \mathbf{l}}(U ; \mathbb{F})$, then $\Theta_{\tau_{0}}(f) \in M_{\mathbf{k}^{\prime}, \mathbf{l}^{\prime}}(U ; \mathbb{F})$. Moreover, $\Theta_{\tau_{0}}(f)$ is divisible by $H_{\theta_{0}}$ if and only if either $f$ is divisible by $H_{\theta_{0}}$ or $p \mid k_{\theta_{0}}$.

Proof. We see exactly as in [12] that $\Theta_{\tau_{0}}(f)$ is regular on the ordinary locus of $\bar{Y}$; that is, the complement of the divisor $\cup_{\theta \in \Sigma} Z_{\theta}$ (where $Z_{\theta}$ was defined in Subsection 4.2), so the theorem reduces to proving that if $z$ is the generic point of an irreducible component of $Z_{\theta_{1}}$ for some $\theta_{1} \in \Sigma$, then

- $\operatorname{ord}_{z}\left(\Theta_{\tau_{0}}(f)\right) \geq 0$,

- if $\theta_{1}=\theta_{0}$, then $\operatorname{ord}_{z}\left(\Theta_{\tau_{0}}(f)\right)>0$ if and only if $p \mid k_{\theta_{0}}$ or $\operatorname{ord}_{z}(f)>0$.

Let $R$ denote the discrete valuation $\operatorname{ring} \mathcal{O}_{\bar{Y}, z}$, and for each $\tau \in \Sigma_{\mathfrak{p}, 0}$ and $\theta \in \Sigma_{\tau}$, let $y_{\theta}=y_{\tau, j}$ be a basis for the stalk $\overline{\mathcal{L}}_{\theta, z}=\overline{\mathcal{L}}_{\tau, j, z}$ over $R$ (for $j=1, \ldots, e_{\mathfrak{p}}$ ). For each $\theta \in \Sigma$, we may then write

$$
H_{\theta} y_{\theta}=r_{\theta} y_{\sigma^{-1} \theta}^{n_{\theta}}
$$

for some $r_{\theta}=r_{\tau, j} \in R$, and we let $r_{\tau}=\prod_{j=1}^{e_{\mathfrak{p}}} r_{\tau, j}$. By construction, we have $T:=$ $\left(\pi_{*} \mathcal{O}_{Y^{\mathrm{Ig}}}\right)_{z}=R\left[x_{\tau}\right]_{\tau \in \Sigma_{0}} / I$, where $I$ is the ideal generated by

$$
x_{\phi^{-1} \circ \tau}^{p}-r_{\tau} x_{\tau} \text { for } \tau \in \Sigma_{0}, \quad \text { and } \prod_{\tau \in \Sigma_{\mathfrak{p}, 0}} x_{\tau}^{p-1}-\prod_{\tau \in \Sigma_{\mathfrak{p}, 0}} r_{\tau}^{p-1} \text { for } \mathfrak{p} \in S_{p},
$$

where each $x_{\tau}$ is the dual basis of $y_{\tau, e_{\mathfrak{p}}}$. We then have $h_{\tau, e_{\mathfrak{p}}}=x_{\tau} y_{\tau, e_{\mathfrak{p}}}\left(\right.$ in $\left.\left(\pi_{*} \pi^{*} \mathcal{L}_{\tau, e_{\mathfrak{p}}}\right)_{z}\right)$, from which it follows that

$$
h_{\tau, j}=r_{\tau, j+1} r_{\tau, j+2} \cdots r_{\tau, e_{p}} x_{\tau} y_{\tau, j}
$$

for $j=1, \ldots, e_{\mathfrak{p}}-1$, and hence that $h^{\mathbf{k}}=\varphi_{\mathbf{k}} y^{\mathbf{k}}$, where $y^{\mathbf{k}}=\prod_{\theta \in \Sigma} y_{\theta}^{k_{\theta}}$ and

$$
\varphi_{\mathbf{k}}=\prod_{\mathfrak{p} \in S_{p}} \prod_{\tau \in \Sigma_{\mathfrak{p}, 0}}\left(\left(r_{\tau} x_{\tau}\right)^{\sum_{\theta \in \Sigma_{\tau}} k_{\theta}} \prod_{j=1}^{e_{\mathfrak{p}}} r_{\tau, j}^{-\sum_{j^{\prime}=j}^{e_{\mathfrak{p}}} k_{\tau, j^{\prime}}}\right)
$$

(writing $k_{\tau_{\mathfrak{p}, i}, j}$ for $k_{\theta_{\mathfrak{p}, i, j}}$ as usual and working over the field of fractions of $T$ ).

Writing $f=\varphi_{f} y^{\mathbf{k}} g^{\mathbf{l}}$, we see that

$$
\Theta_{\tau_{0}}^{\mathrm{Ig}}(f)=K S_{\tau_{0}}^{\mathrm{Ig}}\left(r_{\theta_{0}} \varphi_{\mathbf{k}} d\left(\varphi_{f} \varphi_{\mathbf{k}}^{-1}\right)\right) y_{\theta_{0}}^{-1} y_{\sigma^{-1} \theta_{0}}^{n_{\theta_{0}}} y^{\mathbf{k}} g^{\mathbf{l}}
$$

Since $r_{\tau} x_{\tau}=x_{\phi^{-1} \circ \tau}^{p}$, we have $d\left(r_{\tau} x_{\tau}\right)=0$ and

$$
\Theta_{\tau_{0}}(f)=K S_{\tau_{0}}\left(r_{\theta_{0}} d \varphi_{f}+r_{\theta_{0}} \varphi_{f} \sum_{\theta \in \Sigma} k_{\theta}^{\prime} \frac{d r_{\theta}}{r_{\theta}}\right) y_{\theta_{0}}^{-1} y_{\sigma^{-1} \theta_{0}}^{n_{\theta_{0}}} y^{\mathbf{k}} g^{\mathbf{l}},
$$

where $k_{\theta}^{\prime}=k_{\tau, j}+k_{\tau, j+1}+\cdots k_{\tau, e_{\mathfrak{p}}}$ if $\tau=\tau_{\mathfrak{p}, i}$ and $\theta=\theta_{\mathfrak{p}, i, j}$. We are therefore reduced to showing that $\operatorname{ord}_{z} K S_{\tau_{0}}\left(d r_{\theta_{1}}\right)>0$ if and only if $\theta_{1}=\theta_{0}$. However, the proof of Proposition 4.2.1 shows that if $y$ is a closed point of $Z_{\theta_{1}}$, then $K S_{\tau_{0}}\left(d r_{\theta_{1}}\right)$ vanishes at $y$ if and only if $\theta_{1}=\theta_{0}$.

Remark 5.2.2. The Kodaira-Spencer isomorphism is defined in [12] using the GaussManin connection. Much of the work in $[12, \S 8.2]$ amounts to an explicit translation of 
this to the context of deformation theory. Here, however, we defined the morphism $K S_{\tau_{0}}$ more directly using deformation theory, so the analogue of [12, Lem. 8.2.3] was not needed here.

Remark 5.2.3. It is straightforward to check directly that the right-hand side of (12) is independent of the choice of local trivialisations $y_{\tau}$ and $g_{\tau}$ and can therefore be used to define the partial $\Theta$-operator without reference to the Igusa cover $Y^{\mathrm{Ig}}$.

We call $\Theta_{\tau_{0}}$ the partial $\Theta$-operator (indexed by $\tau_{0}$ ). It is immediate from its definition that the resulting map on $\mathbb{F}$-algebras

$$
\bigoplus_{\mathbf{k}, \mathbf{l} \in \mathbb{Z}^{\Sigma}} M_{\mathbf{k}, \mathbf{l}}(U ; \mathbb{F}) \longrightarrow \bigoplus_{\mathbf{k}, \mathbf{l} \in \mathbb{Z}^{\Sigma}} M_{\mathbf{k}, \mathbf{l}}(U ; \mathbb{F})
$$

given by the direct sum over all weights of the operators $\Theta_{\tau_{0}}$, is an $\mathbb{F}$-linear derivation; that is, that

$$
\Theta_{\tau_{0}}\left(f_{1} f_{2}\right)=f_{1} \Theta_{\tau_{0}}\left(f_{2}\right)+\Theta_{\tau_{0}}\left(f_{1}\right) f_{2}
$$

for all $f_{1}, f_{2}$ in $\oplus M_{\mathbf{k}, \mathbf{l}}(U ; \mathbb{F})$. It is also clear that $\Theta_{\tau_{0}}\left(H_{\theta}\right)=0$ for all $\theta \in \Sigma$, and hence that $\Theta_{\tau_{0}}$ commutes with multiplication by partial Hasse invariants.

It is also straightforward to check that the operator $\Theta_{\tau_{0}}$ commutes with the Hecke action in the obvious sense and hence induces a $\mathrm{GL}_{2}\left(\mathbb{A}_{F, \mathbf{f}}^{(p)}\right)$-equivariant map

$$
\Theta_{\tau}: M_{\mathbf{k}, \mathbf{l}}(\mathbb{F}) \longrightarrow M_{\mathbf{k}^{\prime}, \mathbf{l}^{\prime}}(\mathbb{F})
$$

where $M_{\mathbf{k}, \mathbf{l}}(\mathbb{F})$ (and $M_{\mathbf{k}^{\prime}, \mathbf{l}^{\prime}}(\mathbb{F})$, with their $\mathrm{GL}_{2}\left(\mathbb{A}_{F, \mathbf{f}}^{(p)}\right)$ actions) is defined in (6) as direct limits over suitable open compact $U$.

Let us also make the effect of $\Theta_{\tau_{0}}$ on the weight $\mathbf{k}$ more explicit. Note that if $\mathbf{k}=\sum_{\theta} k_{\theta} \mathbf{e}_{\theta}$ and $\tau_{0}=\tau_{\mathfrak{p}_{0}, i_{0}}$, then $\mathbf{k}^{\prime}=\sum_{\theta} k_{\theta}^{\prime} \mathbf{e}_{\theta}$, where

- if $e_{\mathfrak{p}_{0}}=f_{\mathfrak{p}_{0}}=1$, then $k_{\theta}^{\prime}= \begin{cases}k_{\theta}+p+1, & \text { if } \theta=\theta_{0}=\theta_{\mathfrak{p}_{0}, 1,1}, \\ k_{\theta}, & \text { otherwise }\end{cases}$

- if $e_{\mathfrak{p}_{0}}=1$ and $f_{\mathfrak{p}_{0}}>1$, then $k_{\theta}^{\prime}= \begin{cases}k_{\theta}+1, & \text { if } \theta=\theta_{0}=\theta_{\mathfrak{p}_{0}, i_{0}, 1}, \\ k_{\theta}+p, & \text { if } \theta=\sigma^{-1} \theta_{0}=\theta_{\mathfrak{p}_{0}, i_{0}-1,1}, \\ k_{\theta}, & \text { otherwise; }\end{cases}$

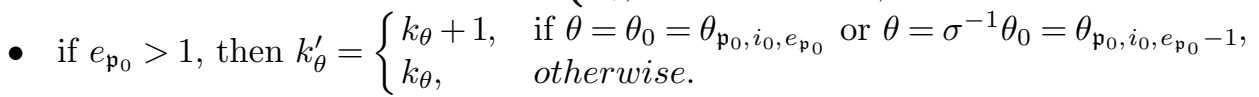

Remark 5.2.4. Considerations from the theory of Serre weights from the point of [12] suggest that the above weight shifts are in a certain sense optimal. One can also define cruder partial $\Theta$-operators by composing with multiplication by (products of) partial Hasse invariants. For example, the operator $H_{\tau_{0}, 1} H_{\tau_{0}, 2} \cdots H_{\tau_{0}, e_{p_{0}}-1} \Theta_{\tau_{0}}$ is the one constructed in [8], and for any $j=1, \ldots, e_{\mathfrak{p}_{0}}$, the operator

$$
H_{\tau_{0}, j} H_{\tau_{0}, j+1}^{2} \cdots H_{\tau_{0}, e_{p_{0}}-1}^{2} H_{\tau_{0}, e_{p_{0}}} \Theta_{\tau_{0}}
$$

shifts the weight $\mathbf{k}$ by $\mathbf{e}_{\theta}+n_{\sigma^{-1} \theta} \mathbf{e}_{\sigma^{-1} \theta}$, where $\theta=\theta_{\mathfrak{p}_{0}, i_{0}, j}$. 


\section{Partial Frobenius operators}

\subsection{Partial Frobenius endomorphisms}

In order to define partial Frobenius operators on Hilbert modular forms (in Subsection 6.2), we first need to define partial Frobenius endomorphisms of Hilbert modular varieties over $\mathbb{F}$.

Fix a prime $\mathfrak{p}$ dividing $p$ and a level $U$, assumed as usual to be sufficiently small and prime to $p$. We will draw on ideas from $[11, \S 7.1]$ to construct an isogeny on the universal abelian variety $s: A \rightarrow S$, where $S=\widetilde{Y}_{U, \mathbb{F}}$.

We begin by associating Raynaud data to the line bundles $\mathcal{L}_{\mathfrak{p}, i, e_{\mathfrak{p}}}$ over $S$, which we write simply as $\mathcal{L}_{i}$ for $i \in \mathbb{Z} / f \mathbb{Z}=\mathbb{Z} / f_{\mathfrak{p}} \mathbb{Z}$ (omitting the subscripts for the fixed $\mathfrak{p}$ and $\left.j=e=e_{\mathfrak{p}}\right)$. We define $f_{i}: \mathcal{L}_{i}^{\otimes p} \rightarrow \mathcal{L}_{i+1}$ to be zero, and we define $v_{i}: \mathcal{L}_{i+1} \rightarrow \mathcal{L}_{i}^{\otimes p}$ to be the morphism induced by the restriction of

$$
\operatorname{Ver}_{A}^{*}: \mathcal{H}=\mathcal{H}_{\mathrm{dR}}^{1}(A / S)_{\mathfrak{p}} \rightarrow \mathcal{H}_{\mathrm{dR}}^{1}\left(\left(\phi_{S}^{*} A\right) / S\right)_{\mathfrak{p}}=\phi_{S}^{*} \mathcal{H}_{\mathrm{dR}}^{1}(A / S)_{\mathfrak{p}}=\phi_{S}^{*} \mathcal{H}
$$

to $\mathcal{F}_{i}^{(e)}=\left(s_{*} \Omega_{A / S}^{1}\right)_{i}$ (abbreviating subscripts $\tau_{\mathfrak{p}, i}$ by $i$ ). Note that since the image of $\mathcal{H}_{i+1}$ under $\operatorname{Ver}_{A}^{*}$ is $\phi_{S}^{*}\left(\mathcal{F}_{i}^{(e)}\right)$, the inclusions $\mathcal{F}_{i+1}^{(e-1)} \subset u \mathcal{H}_{i+1}$ and $u \mathcal{F}_{i}^{(e)} \subset \mathcal{F}_{i}^{(e-1)}$ ensure that $\operatorname{Ver}_{A}^{*}\left(\mathcal{F}_{i+1}^{(e-1)}\right) \subset \phi_{S}^{*}\left(\mathcal{F}_{i}^{(e-1)}\right)$, so the morphism $v_{i}$ is well-defined. We then let $H$ denote the finite flat $\left(\mathcal{O}_{F} / \mathfrak{p}\right)$-vector space scheme over $S$ associated to the Raynaud

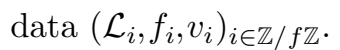

Recall that the Dieudonné crystal of $\operatorname{ker}\left(\mathrm{Frob}_{A}\right)$ is canonically isomorphic to $\Phi^{*}\left(s_{*} \Omega_{A / S}^{1}\right)$, with $F=0$ and $V$ induced by $\Phi^{*}\left(\operatorname{Ver}_{A}^{*}\right)$ (in the notation of $[2, \S 4.4 .3]$ ). On the other hand, the Dieudonné crystal of $H$ is identified with $\Phi^{*}\left(\oplus_{i} \mathcal{L}_{i}\right)$ with $F=\Phi^{*}\left(\oplus_{i} f_{i}\right)=0$ and $V=\Phi^{*}\left(\oplus_{i} v_{i}\right)$ (as a simple special case of [11, Prop. 7.1.3]). Therefore, the canonical projection $s_{*} \Omega_{A / S}^{1} \rightarrow \oplus_{i} \mathcal{L}_{i}$ induces a surjective morphism of Dieudonné crystals $\mathbb{D}\left(\operatorname{ker}\left(\operatorname{Frob}_{A}\right)\right) \rightarrow \mathbb{D}(H)$. As the base $S$ is smooth over $\mathbb{F}$, the exact contravariant functor $\mathbb{D}$ is fully faithful on finite flat $p$-group schemes over $S$ ([3, Thm. 4.1.1]), so the surjection arises from a closed immersion $H \hookrightarrow \operatorname{ker}\left(\operatorname{Frob}_{A}\right)$, and we let

$$
\alpha: A \longrightarrow A^{\prime}:=A / H
$$

denote the resulting isogeny of abelian varieties over $S$. Note that $A^{\prime}$ naturally inherits an $\mathcal{O}_{F}$-action $\iota^{\prime}$ from the action $\iota$ on $A$.

Let $\mathcal{I}$ denote the image of the morphism $\alpha^{*}: \mathcal{H}_{\mathrm{dR}}^{1}\left(A^{\prime} / \widetilde{Y}_{U, \mathbb{F}}\right)_{\mathfrak{p}} \rightarrow \mathcal{H}$. By construction, we have the exact sequence

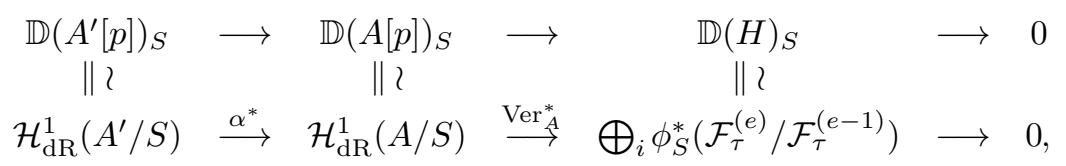

showing that $\mathcal{I}=\oplus_{i} \mathcal{I}_{i}$, where $\mathcal{I}_{i}$ is the pre-image of $\phi_{S}^{*}\left(\mathcal{F}_{i-1}^{(e)}\right)$ under $\operatorname{Ver}_{A, i}^{*}: \mathcal{H}_{i} \rightarrow$ $\phi_{S}^{*}\left(\mathcal{H}_{i-1}\right)$. Note in particular that $u \mathcal{H}_{i} \subset \mathcal{I}_{i}$ for all $i$, so that $H \subset A[\mathfrak{p}]$ and there is a unique isogeny $\beta: \mathfrak{p} \otimes_{\mathcal{O}_{F}} A^{\prime} \longrightarrow A$ such that $\alpha \circ \beta$ is the canonical isogeny $\mathfrak{p} \otimes_{\mathcal{O}_{F}} A^{\prime} \rightarrow A^{\prime}$.

We now equip $A^{\prime}$ with auxiliary data corresponding to an element of $\widetilde{Y}_{U, \mathbb{F}}(S)$. 
Since $\alpha$ induces isomorphisms $T^{(p)}\left(A_{\bar{s}}\right) \stackrel{\sim}{\longrightarrow} T^{(p)}\left(A_{\bar{s}}^{\prime}\right)$ for all geometric points $\bar{s}$ of $S$, we immediately have a level $U^{p}$ structure $\eta^{\prime}$ on $A^{\prime}$ inherited from $A$.

Next we claim that the quasi-polarisation $\lambda$ on $A$ induces an isomorphism ${ }^{10} \mathfrak{p c d} \otimes \mathcal{O}_{F}$ $A^{\prime} \rightarrow\left(A^{\prime}\right)^{\vee}$ or, equivalently, $A^{\prime} \rightarrow \mathfrak{p}^{-1} \mathfrak{c}^{-1} \mathfrak{d}^{-1} \otimes_{\mathcal{O}_{F}}\left(A^{\prime}\right)^{\vee}$, which amounts to the claim that $H$ corresponds to the kernel of

$$
\mathfrak{c}^{-1} \mathfrak{d}^{-1} \otimes \beta^{\vee}: \mathfrak{c}^{-1} \mathfrak{d}^{-1} \otimes_{\mathcal{O}_{F}} A^{\vee} \longrightarrow \mathfrak{p}^{-1} \mathfrak{c}^{-1} \mathfrak{d}^{-1} \otimes_{\mathcal{O}_{F}}\left(A^{\prime}\right)^{\vee}
$$

under the isomorphism induced by $\lambda$. Denoting this kernel by $I$, we have that $H$ and $I$ are finite flat group schemes over $S$ of the same rank, so it suffices to prove that the composite

$$
I \longrightarrow \mathfrak{c}^{-1} \mathfrak{d}^{-1} \otimes_{\mathcal{O}_{F}} A^{\vee}[p] \stackrel{\sim}{\longrightarrow} A[p] \longrightarrow A^{\prime}[p]
$$

is trivial. Taking Dieudonné modules, this in turn amounts to the vanishing of the composite

$$
\mathbb{D}\left(A^{\prime}[p]\right)_{S} \longrightarrow \mathbb{D}(A[p])_{S} \longrightarrow \mathbb{D}\left(\mathfrak{c}^{-1} \mathfrak{d}^{-1} \otimes \mathcal{O}_{F} A^{\vee}[p]\right)_{S} \longrightarrow \mathbb{D}(I)_{S}
$$

We have already noted that the image of the first map has $\mathfrak{p}$-component $\oplus_{i} \mathcal{I}_{i}$; on the other hand, the kernel of the last map is the image of the map

$$
\mathbb{D}\left(\mathfrak{p}^{-1} \mathfrak{c}^{-1} \mathfrak{d}^{-1} \otimes_{\mathcal{O}_{F}}\left(A^{\prime}\right)^{\vee}[p]\right)_{S} \longrightarrow \mathbb{D}\left(\mathfrak{c}^{-1} \mathfrak{d}^{-1} \otimes_{\mathcal{O}_{F}} A^{\vee}[p]\right)_{S}
$$

corresponding to the adjoint of

$$
\beta^{*}: \mathcal{H}_{\mathrm{dR}}^{1}(A / S) \rightarrow \mathcal{H}_{\mathrm{dR}}^{1}\left(\left(\mathfrak{p} \otimes_{\mathcal{O}_{F}} A^{\prime}\right) / S\right) \cong \mathfrak{p}^{-1} \otimes_{\mathcal{O}_{F}} \mathcal{H}_{\mathrm{dR}}^{1}\left(A^{\prime} / S\right)
$$

under the canonical isomorphisms

$$
\begin{aligned}
\mathbb{D}\left(\mathfrak{c}^{-1} \mathfrak{d}^{-1} \otimes_{\mathcal{O}_{F}} A^{\vee}[p]\right)_{S} \cong \mathcal{H}_{\mathrm{dR}}^{1}\left(\left(\mathfrak{c}^{-1} \mathfrak{d}^{-1} \otimes_{\mathcal{O}_{F}} A^{\vee} / S\right)\right. & \\
& \cong \mathcal{H} o m_{\mathcal{O}_{S}}\left(\mathfrak{d}^{-1} \otimes_{\mathcal{O}_{F}} \mathcal{H}_{\mathrm{dR}}^{1}(A / S), \mathcal{O}_{S}\right) \cong \mathcal{H} m_{\mathcal{O}_{F} \otimes \mathcal{O}_{S}}\left(\mathcal{H}_{\mathrm{dR}}^{1}(A / S), \mathcal{O}_{F} \otimes \mathcal{O}_{S}\right)
\end{aligned}
$$

and, similarly,

$$
\mathbb{D}\left(\mathfrak{p}^{-1} \mathfrak{c}^{-1} \mathfrak{d}^{-1} \otimes_{\mathcal{O}_{F}}\left(A^{\prime}\right)^{\vee}[p]\right)_{S} \cong \mathcal{H} o m_{\mathcal{O}_{F} \otimes \mathcal{O}_{S}}\left(\mathfrak{p}^{-1} \otimes_{\mathcal{O}_{F}} \mathcal{H}_{\mathrm{dR}}^{1}\left(A^{\prime} / S\right), \mathcal{O}_{F} \otimes \mathcal{O}_{S}\right)
$$

obtained from duality. We are therefore reduced to proving that $\mathcal{I}_{i}$ is orthogonal to the kernel of $\beta_{i}^{*}$ for each $i \in \mathbb{Z} / f \mathbb{Z}$ under the pairing $\langle\cdot, \cdot\rangle_{i}$ defined by (4). Note, however, that the kernel of $\beta_{i}^{*}$ is $u^{e-1} \mathcal{I}_{i}$, as can be seen, for example, from the commutative diagram

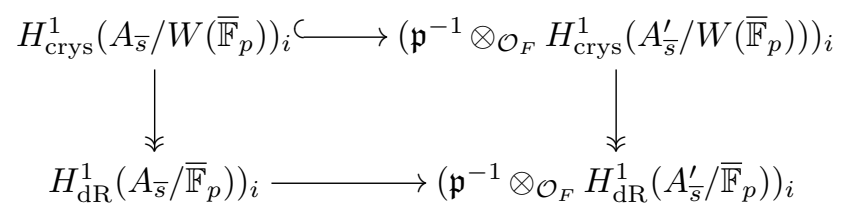

of $W\left(\overline{\mathbb{F}}_{p}\right)[u]$-modules for $\bar{s} \in S\left(\overline{\mathbb{F}}_{p}\right)$. Finally, the orthogonality of $\mathcal{I}_{i}$ and $u^{e-1} \mathcal{I}_{i}$ is immediate from that of $\mathcal{F}_{i-1}^{(e-1)}$ and $u^{-1} \mathcal{F}_{i-1}^{(e-1)}$ provided by Lemma 3.1.1, completing the proof of the claim. We may then define the quasi-polarisation on $A^{\prime}$ by $\alpha^{*}\left(\lambda^{\prime}\right)=\delta \lambda$

${ }^{10}$ Here $\mathfrak{c}$ depends on the connected component of $S$. 
for any totally positive generator $\delta=\delta_{\mathfrak{p}}$ of $\mathfrak{p} \mathcal{O}_{F,(p)}$, so that $\lambda^{\prime}$ induces an isomorphism $\mathfrak{c}^{\prime} \mathfrak{d} \otimes \mathcal{O}_{F} A^{\prime} \stackrel{\sim}{\longrightarrow}\left(A^{\prime}\right)^{\vee}$ where $\mathfrak{c}^{\prime}=\delta^{-1} \mathfrak{p c}$.

Finally, we define a Pappas-Rapoport filtration on $\mathcal{F}_{\tau}^{\prime}:=\left(s_{*}^{\prime} \Omega_{A^{\prime} / S}^{1}\right)_{\tau}$ for all $\tau \in \Sigma_{0}$.

First, note that if $\tau \notin \Sigma_{\mathfrak{p}, 0}$, then $\alpha^{*}$ induces an isomorphism $\mathcal{F}_{\tau}^{\prime} \simeq \mathcal{F}_{\tau}^{(e)}$, and we define $\left(\mathcal{F}_{\tau}^{\prime}\right)^{(j)}$ as the pre-image of $\mathcal{F}_{\tau}^{(j)}$.

Suppose now that $\tau=\tau_{\mathfrak{p}, i}$. Recall from the construction of $A^{\prime}=A / H$ that $\operatorname{Ver}_{A}^{*}\left(\mathcal{F}_{i}^{(e-1)}\right) \subset \phi_{S}^{*}\left(\mathcal{F}_{i-1}^{(e-1)}\right)$, so we have $\mathcal{F}_{i}^{(e-1)} \subset \mathcal{I}_{i}$. It follows that $\left(\alpha_{i}^{*}\right)^{-1}\left(\mathcal{F}_{i}^{(e-1)}\right)$ is a subbundle of $\mathcal{H}_{i}^{\prime}:=\mathcal{H}_{\mathrm{dR}}^{1}\left(A^{\prime}\right)_{i}$ of the same rank as $\mathcal{F}_{i}^{\prime}$, namely, e. Furthermore, we have

$$
\begin{aligned}
\phi_{S}^{*}\left(\alpha^{*}\left(\mathcal{F}_{i}^{\prime}\right)\right)=\left(\phi_{S}^{*}(\alpha)\right)^{*}\left(\mathcal{F}_{i}^{\prime}\right) & =\left(\phi_{S}^{*}(\alpha)\right)^{*}\left(\operatorname{Ver}_{A^{\prime}}^{*}\left(\mathcal{H}_{i+1}^{\prime}\right)\right) \\
& =\operatorname{Ver}_{A}^{*}\left(\alpha^{*}\left(\mathcal{H}_{i+1}^{\prime}\right)\right)=\operatorname{Ver}_{A}^{*}\left(\mathcal{I}_{i+1}\right) \subset \phi_{S}^{*}\left(\mathcal{F}_{i}^{(e+1)}\right),
\end{aligned}
$$

so in fact $\mathcal{F}_{i}^{\prime} \subset\left(\alpha_{i}^{*}\right)^{-1}\left(\mathcal{F}_{i}^{(e-1)}\right)$, and hence equality holds. We thus obtain an exact sequence

$$
0 \rightarrow \operatorname{ker}\left(\alpha_{i}^{*}\right) \longrightarrow \mathcal{F}_{i}^{\prime} \stackrel{\alpha_{i}^{*}}{\longrightarrow} \mathcal{F}_{i}^{(e-1)} \rightarrow 0 .
$$

We may thus define a Pappas-Rapoport filtration on $\mathcal{F}_{i}^{\prime}$ by setting

$$
\left(\mathcal{F}_{i}^{\prime}\right)^{(j)}=\left(\alpha_{i}^{*}\right)^{-1}\left(\mathcal{F}_{i}^{(j-1)}\right)
$$

for $j=1, \ldots, e$, so, in particular, $\left(\mathcal{F}_{i}^{\prime}\right)^{(1)}=\operatorname{ker}\left(\alpha_{i}^{*}\right)$.

We now define $\widetilde{\Phi}_{\mathfrak{p}}: \widetilde{Y}_{U, \mathbb{F}}=S \rightarrow \widetilde{Y}_{U, \mathbb{F}}$ to be the endomorphism corresponding to the data $\left(A^{\prime}, \iota^{\prime}, \lambda^{\prime}, \eta^{\prime},\left(\mathcal{F}^{\prime}\right)^{\bullet}\right)$. Note that $\widetilde{\Phi}_{\mathfrak{p}}$ depends on the choice of $\delta$ in the definition of $\lambda^{\prime}$; however, it is straightforward to check that $\widetilde{\Phi}_{\mathfrak{p}}$ is compatible with the $\mathcal{O}_{F,(p),+}^{\times}$-action on $S$ and descends to an endomorphism $\Phi_{\mathfrak{p}}$ of $\bar{Y}_{U}$ which is independent of this choice. We call $\widetilde{\Phi}_{\mathfrak{p}}$ (respectively $\Phi_{\mathfrak{p}}$ ) the partial Frobenius endomorphism (indexed by $\mathfrak{p}$ ) of $\widetilde{Y}_{U, \mathbb{F}}$ (respectively $\bar{Y}_{U}$ ); the terminology is justified by the next proposition.

For the statement of the proposition, we also define the endomorphism $\widetilde{\Phi}$ of $S=\widetilde{Y}_{U, \mathbb{F}}$ corresponding to the data $\phi_{S}^{*}(\underline{A})=\left(\phi_{S}^{*} A, \phi_{S}^{*} \iota, \phi_{S}^{*} \lambda, \phi_{S}^{*} \eta,\left(\phi_{S}^{*} \mathcal{F}\right)^{\bullet}\right)$, where $\left(\phi_{S}^{*} \mathcal{F}\right)^{\bullet}$ is the collection of filtrations on the vector bundles

$$
\left(\left(\phi_{S}^{*} s\right)_{*}\left(\Omega_{\left(\phi_{S}^{*} A\right) / S}^{1}\right)\right)_{\tau}=\left(\phi_{S}^{*}\left(s_{*} \Omega_{A / S}^{1}\right)\right)_{\tau}=\phi_{S}^{*}\left(\left(s_{*} \Omega_{A / S}^{1}\right)_{\phi^{-1} \circ \tau}\right)
$$

given by $\left(\phi_{S}^{*} \mathcal{F}\right)_{\tau}^{(j)}=\phi_{S}^{*}\left(\mathcal{F}_{\phi^{-1} \circ \tau}^{(j)}\right)$. Note that $\widetilde{\Phi}$ is not the absolute Frobenius $\phi_{S}$ on $S$ (unless $\mathbb{F}=\mathbb{F}_{p}$ ), but we may write $\phi_{S}=\widetilde{\Phi} \circ \widetilde{\epsilon}$ where $\widetilde{\epsilon}$ is the isomorphism defined by the commutative diagram

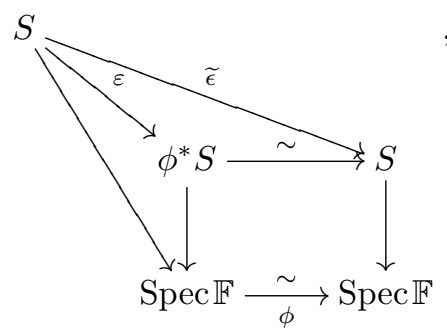


where the square is Cartesian and $\varepsilon$ is the inverse of the isomorphism associated to $\phi^{*} A=A \times_{\mathbb{F}, \phi} \mathbb{F}$ with the evident auxiliary data. We thus have an isomorphism $\widetilde{\epsilon}^{*} A \cong A$ compatible with $\iota, \lambda$ and $\eta$ and inducing $\widetilde{\epsilon}^{*} \mathcal{F}_{\tau}^{(j)} \cong \mathcal{F}_{\phi \circ \tau}^{(j)}$ for all $\tau$ and $j$. (Note also that $\widetilde{\Phi}$ may be viewed as the base change of the absolute Frobenius on the descent of $S$ to $\mathbb{F}_{p}$ defined by the diagram.)

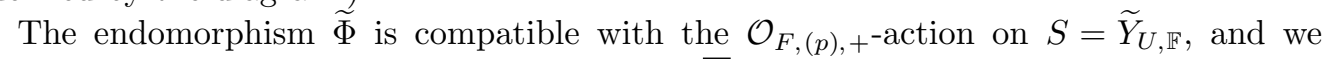
let $\Phi$ denote the resulting endomorphism of $\bar{Y}_{U}$. Similarly, $\widetilde{\epsilon}$ descends to a $\phi$-linear automorphism $\epsilon$ of $\bar{Y}_{U}$ such that the absolute Frobenius on $\bar{Y}_{U}$ is $\Phi \circ \epsilon$.

Proposition 6.1.1. The morphisms $\Phi_{\mathfrak{p}}$ are finite flat of (constant) degree $\mathrm{Nm}_{F / \mathbb{Q}}(\mathfrak{p})$, commute with each other and satisfy the formula

$$
\prod_{\mathfrak{p} \in S_{p}} \Phi_{\mathfrak{p}}^{e_{\mathfrak{p}}}=\Phi .
$$

Proof. We first prove the commutativity and analogous formula for the maps $\widetilde{\Phi}_{\mathfrak{p}}$ on $S=\widetilde{Y}_{U, \mathbb{F}}$, from which the corresponding assertions for $\Phi_{\mathfrak{p}}$ follow. To that end, it suffices to consider the maps on geometric closed points $\bar{s} \in S\left(\overline{\mathbb{F}}_{p}\right)$, which we will do in order to facilitate computations on Dieudonné modules.

Let $\underline{A}_{0}$ denote the data corresponding to $\bar{s} \in S\left(\overline{\mathbb{F}}_{p}\right)$ and $\underline{A}_{0}^{\prime}=\underline{A}_{0, \mathfrak{p}}^{\prime}$ denote the data corresponding to $\widetilde{\Phi}_{\mathfrak{p}}(\bar{s})$. Let $D=H_{\text {crys }}^{1}\left(A_{0} / W\left(\overline{\mathbb{F}}_{p}\right)\right)$ and $D^{\prime}=H_{\text {crys }}^{1}\left(A_{0}^{\prime} / W\left(\overline{\mathbb{F}}_{p}\right)\right)$, so we may decompose the $\mathcal{O}_{F} \otimes W\left(\overline{\mathbb{F}}_{p}\right)$-modules $D=\oplus_{\tau \in \Sigma} D_{\tau}$ and $D^{\prime}=\oplus_{\tau \in \Sigma^{\prime}} D_{\tau}^{\prime}$ where $D_{\tau}$ and $D_{\tau}^{\prime}$ are free $W\left(\overline{\mathbb{F}}_{p}\right)[u] /\left(f^{\tau}(u)\right)$-modules of rank 2 . Furthermore, the canonical isogeny $\alpha: A_{0} \rightarrow A_{0}^{\prime}$ induces an injective $W\left(\overline{\mathbb{F}}_{p}\right)[u] /\left(f^{\tau}(u)\right)$-linear map $\alpha_{\tau}^{*}: D_{\tau}^{\prime} \rightarrow D_{\tau}$ for each $\tau$, compatible in the obvious sense with the maps

$$
\operatorname{Frob}_{A_{0}, \tau}^{*}: \phi^{*}\left(D_{\phi^{-1} \circ \tau}\right) \rightarrow D_{\tau} \quad \text { and } \operatorname{Frob}_{A_{0}^{\prime}, \tau}^{*}: \phi^{*}\left(D_{\phi^{-1} \circ \tau}^{\prime}\right) \rightarrow D_{\tau}^{\prime},
$$

as well as $\operatorname{Ver}_{A_{0}, \tau}^{*}=p\left(\operatorname{Frob}_{A_{0}, \tau}^{*}\right)^{-1}$ and $\operatorname{Ver}_{A_{0}^{\prime}, \tau}^{*}=p\left(\operatorname{Frob}_{A_{0}^{\prime}, \tau}^{*}\right)^{-1}$. Letting $F_{\tau}^{(j)} \subset D_{\tau} \operatorname{denote}$ the pre-image of $\mathcal{F}_{\tau}^{(j)}$ under the canonical surjection

$$
D_{\tau} \longrightarrow(D / p D)_{\tau} \cong H_{\mathrm{dR}}^{1}\left(A_{0} / \overline{\mathbb{F}}_{p}\right)_{\tau}
$$

we have $\alpha_{\tau}^{*}\left(D_{\tau}^{\prime}\right)=D_{\tau}$ if $\tau \notin \Sigma_{\mathfrak{p}, 0}$ and

$$
\alpha_{\tau}^{*}\left(D_{\tau}^{\prime}\right)=\left(\operatorname{Ver}_{A_{0}, \tau}^{*}\right)^{-1}\left(\phi^{*}\left(F_{\phi^{-1} \circ \tau}^{\left(e_{\mathfrak{p}}-1\right)}\right)\right)
$$

if $\tau \in \Sigma_{\mathfrak{p}, 0}$ (by the construction of $\Phi_{\mathfrak{p}}$ ). Furthermore, writing $F_{\tau}^{\prime(j)}$ for the submodules of $D_{\tau}^{\prime}$ similarly defined by the Pappas-Rapoport filtration on $H^{0}\left(A_{0}^{\prime}, \Omega_{A_{0}^{\prime} / \overline{\mathbb{F}}_{p}}^{1}\right)_{\tau}$, we have

- $\alpha_{\tau}^{*}\left(F_{\tau}^{\prime(j)}\right)=F_{\tau}^{(j)}$ if $\tau \notin \Sigma_{\mathfrak{p}, 0}$,

- $\alpha_{\tau}^{*}\left(F_{\tau}^{\prime(j)}\right)=F_{\tau}^{(j-1)}$ for $j=2, \ldots, e_{\mathfrak{p}}$ if $\tau \in \Sigma_{\mathfrak{p}, 0}$,

- $\quad$ and $\alpha_{\tau}^{*}\left(F_{\tau}^{\prime(1)}\right)=p D_{\tau}=\operatorname{Frob}_{A_{0}, \tau}^{*}\left(\phi^{*}\left(F_{\phi^{-1} \circ \tau}^{\left(e_{\mathfrak{p}}\right)}\right)\right)$ if $\tau \in \Sigma_{\mathfrak{p}, 0}$.

Thus, if $\mathfrak{p}_{1}$ and $\mathfrak{p}_{2}$ are distinct elements of $S_{p}$, then $\widetilde{\Phi}_{\mathfrak{p}_{1}}\left(\widetilde{\Phi}_{\mathfrak{p}_{2}}(\bar{s})\right)$ corresponds to the data $\underline{A}_{0}^{\prime \prime}$ for which we have an isogeny $\alpha^{\prime}: A_{0} \rightarrow A_{0}^{\prime \prime}$ such that if $\tau \notin \Sigma_{\mathfrak{p}_{1}, 0} \cup \Sigma_{\mathfrak{p}_{2}, 0}$ then 
$\left(\alpha^{\prime}\right)_{\tau}^{*}\left(D_{\tau}^{\prime \prime}\right)=D_{\tau}$ and $\left(\alpha^{\prime}\right)_{\tau}^{*}\left(F_{\tau}^{\prime \prime(j)}\right)=F_{\tau}^{(j)}$ (with the obvious notation), but if $\tau \in \Sigma_{\mathfrak{p}_{i}, 0}$ for $i=1$ or 2 , then

$$
\left(\alpha^{\prime}\right)_{\tau}^{*}\left(D_{\tau}^{\prime \prime}\right)=\left(\operatorname{Ver}_{A_{0}, \tau}^{*}\right)^{-1}\left(\phi^{*}\left(F_{\phi^{-1} \circ \tau}^{\left(e_{\mathfrak{p}_{i}}-1\right)}\right)\right)
$$

$\left(\alpha^{\prime}\right)_{\tau}^{*}\left(F_{\tau}^{\prime \prime(1)}\right)=p D_{\tau}$ and $\left(\alpha^{\prime}\right)_{\tau}^{*}\left(F_{\tau}^{\prime \prime(j)}\right)=F_{\tau}^{(j-1)}$ for $j=2, \ldots, e_{\mathfrak{p}_{i}}$. Furthermore, we have $\eta^{\prime \prime}=\alpha^{\prime} \circ \eta$ and $\left(\alpha^{\prime}\right)^{*}\left(\lambda^{\prime \prime}\right)=\delta_{\mathfrak{p}_{2}} \delta_{\mathfrak{p}_{1}} \lambda$, from which it follows that the isomorphism class of the data $\underline{A}_{0}^{\prime \prime}$ also corresponds to $\widetilde{\Phi}_{\mathfrak{p}_{2}}\left(\widetilde{\Phi}_{\mathfrak{p}_{1}}(\bar{s})\right)$.

Now consider the data $\underline{A}_{0}^{(r)}$ associated to $\widetilde{\Phi}_{\mathfrak{p}}^{r}(\bar{s})$ for $r=1, \ldots, e_{\mathfrak{p}}$, and write $D_{r}=\oplus D_{r, \tau}$ for $H_{\text {crys }}^{1}\left(A_{0}^{(r)} / W\left(\overline{\mathbb{F}}_{p}\right)\right), F_{r, \tau}$ for the submodule of $D_{r, \tau}$ determined as above by the Pappas-Rapoport filtration, and $\alpha_{r}$ for the composite isogeny $A_{0} \rightarrow A_{0}^{(1)} \rightarrow \cdots \rightarrow A_{0}^{(r)}$. By induction on $r$, we find that if $\tau \notin \Sigma_{\mathfrak{p}, 0}$, then $\alpha_{r, \tau}^{*}\left(D_{r, \tau}\right)=D_{\tau}$ and $\alpha_{r, \tau}^{*}\left(F_{r, \tau}^{(j)}\right)=F_{\tau}^{(j)}$, but if $\tau \in \Sigma_{\mathfrak{p}, 0}$, then

$$
\begin{array}{ll}
\text { - } & \alpha_{r, \tau}^{*}\left(D_{r, \tau}\right)=\left(\operatorname{Ver}_{A_{0}, \tau}^{*}\right)^{-1}\left(\phi^{*}\left(F_{\phi^{-1} \circ \tau}^{\left(e_{\mathfrak{p}}-r\right)}\right)\right), \\
\text { - } & \alpha_{r, \tau}^{*}\left(F_{r, \tau}^{(j)}\right)=F_{\tau}^{(j-r)} \text { for } j=r+1, \ldots, e_{\mathfrak{p}}, \\
\text { - } & \alpha_{r, \tau}^{*}\left(F_{r, \tau}^{(j)}\right)=\operatorname{Frob}_{A_{0}, \tau}^{*}\left(\phi^{*}\left(F_{\phi^{-1} \circ \tau}^{\left(e_{\mathfrak{p}}+j-r\right)}\right)\right) \text { for } j=1, \ldots, r .
\end{array}
$$

In particular, taking $r=e_{\mathfrak{p}}$ gives

$$
\alpha_{e_{\mathfrak{p}}, \tau}^{*}\left(D_{e_{\mathfrak{p}}, \tau}\right)=\left(\operatorname{Ver}_{A_{0}, \tau}^{*}\right)^{-1}\left(p \phi^{*}\left(D_{\phi^{-1} \circ \tau}\right)\right)=\operatorname{Frob}_{A_{0}, \tau}^{*}\left(\phi^{*}\left(D_{\phi^{-1} \circ \tau}\right)\right)
$$

and $\alpha_{e_{\mathfrak{p}}, \tau}^{*}\left(F_{e_{\mathfrak{p}}, \tau}^{(j)}\right)=\operatorname{Frob}_{A_{0}, \tau}^{*}\left(\phi^{*}\left(F_{\phi^{-1} \circ \tau}^{(j)}\right)\right)$ for $j=1, \ldots, e_{\mathfrak{p}}$ and $\tau \in \Sigma_{\mathfrak{p}, 0}$. It then follows that $\prod_{\mathfrak{p} \in S_{p}} \widetilde{\Phi}_{\mathfrak{p}}^{e_{\mathfrak{p}}}(\bar{s})$ corresponds to $\underline{A}_{0}^{\prime \prime}$ with $\alpha^{\prime}: A_{0} \rightarrow A_{0}^{\prime \prime}$ satisfying $\left(\alpha^{\prime}\right)_{\tau}^{*}\left(D_{\tau}^{\prime \prime}\right)=$ $\operatorname{Frob}_{A_{0}, \tau}^{*}\left(\phi^{*}\left(D_{\phi^{-1} \circ \tau}\right)\right)$ and $\left(\alpha^{\prime}\right)_{\tau}^{*}\left(F_{\tau}^{\prime \prime(j)}\right)=\operatorname{Frob}_{A_{0}, \tau}^{*}\left(\phi^{*}\left(F_{\phi^{-1} \circ \tau}^{(j)}\right)\right)$ for all $\tau$ and $j$. Furthermore, we have $\eta^{\prime \prime}=\alpha^{\prime} \circ \eta$ and $\left(\alpha^{\prime}\right)^{*}\left(\lambda^{\prime \prime}\right)=\prod_{\mathfrak{p}} \delta_{\mathfrak{p}}^{e_{\mathfrak{p}}} \lambda$, from which it follows that $\underline{A}_{0}^{\prime \prime}$ is isomorphic to $\left(\phi^{*} A_{0}, \phi^{*} \iota, \nu \phi^{*} \lambda,\left(\phi^{*} \mathcal{F}\right)^{\bullet}\right)$ with $\nu=p^{-1} \prod_{\mathfrak{p}} \delta_{\mathfrak{p}}^{e_{\mathfrak{p}}} \in \mathcal{O}_{F,(p),+}^{\times}$. This proves that

$$
\prod_{\mathfrak{p} \in S_{p}} \widetilde{\Phi}_{\mathfrak{p}}^{e_{\mathfrak{p}}}=\nu \cdot \widetilde{\Phi},
$$

which in turn implies the desired formula.

Since $\Phi$ is finite (and $\bar{Y}_{U}$ is separated), it follows that $\Phi_{\mathfrak{p}}$ is finite and therefore also flat since $\bar{Y}_{U}$ regular. Note furthermore that $\Phi_{\mathfrak{p}}$ is therefore bijective on closed points and induces isomorphisms on their residue fields, so the degree of $\Phi_{\mathfrak{p}}$ in a neighbourhood of any closed point $x$ of $\bar{Y}_{U}$ is that of the extension of completed regular local rings

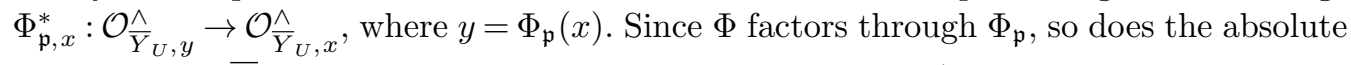
Frobenius on $\bar{Y}_{U}$, and hence the absolute Frobenius on $\mathcal{O}_{\bar{Y}_{U}, x}^{\wedge}$ factors through $\Phi_{\mathfrak{p}, x}^{*}$. Therefore, it follows from [26, Cor. 2] that $\operatorname{deg}\left(\Phi_{\mathfrak{p}, x}^{*}\right)=p^{n}$ where $n=n_{\mathfrak{p}}$ is the dimension of the kernel of the induced map on tangent spaces $T_{x}\left(\bar{Y}_{U}\right) \rightarrow T_{y}\left(\bar{Y}_{U}\right)$. Furthermore, since $\prod_{\mathfrak{p} \in S_{p}} \Phi_{\mathfrak{p}}^{e_{\mathfrak{p}}}=\Phi$ has degree $p^{[F: \mathbb{Q}]}=\prod_{\mathfrak{p} \in S_{p}} p^{e_{\mathfrak{p}} f_{\mathfrak{p}}}$, it suffices to prove that $n_{\mathfrak{p}} \geq f_{\mathfrak{p}}=$ $N m_{F / \mathbb{Q}}(\mathfrak{p})$ for each $\mathfrak{p}$. Note also that we may replace $\bar{Y}_{U}$ by $S=\widetilde{Y}_{U, \mathbb{F}}, x$ by any point in its pre-image in $S$ and $\Phi_{\mathfrak{p}}$ by $\widetilde{\Phi}_{\mathfrak{p}}$. 
Suppose then that $x$ corresponds to the data $\left(A_{0}, \iota_{0}, \lambda_{0}, \eta_{0}, \mathcal{F}_{0}^{\bullet}\right)$ over the residue field $k$ and its image $y=\widetilde{\Phi}_{\mathfrak{p}}(x)$ corresponds to the data $\left(A_{0}^{\prime}, \iota_{0}^{\prime}, \lambda_{0}^{\prime}, \eta_{0}^{\prime}, \mathcal{F}_{0}^{\prime \bullet}\right)$. Recall that the Kodaira-Spencer filtration on the fibre of $\Omega_{S / \mathbb{F}_{p}}^{1}$ at $x$ is dual to one on $T_{x}(S)$ which was described using Grothendieck-Messing deformation theory (see the discussion following the proof of Theorem 3.3.1). In particular, we have a decomposition $T_{x}(S)=\oplus_{\tau \in \Sigma_{0}} T_{x}(S)_{\tau}$ and a decreasing filtration of length $e_{\mathfrak{p}^{\prime}}$ on $T_{x}(S)_{\tau}$ for each $\tau \in \Sigma_{\mathfrak{p}^{\prime}, 0}$ (where $\mathfrak{p}^{\prime} \in S_{p}$ ) such that

$$
\bigoplus_{\tau \in \Sigma_{0}} \mathrm{Fil}^{j_{\tau}} T_{x}(S)_{\tau}
$$

corresponds to the set of lifts of $\underline{A}_{0}$ to $\underline{A}_{1}=\left(A_{1}, \iota_{1}, \lambda_{1}, \eta_{1}, \mathcal{F}_{1}^{\bullet}\right) \in S(k[\epsilon])$ such that $\mathcal{F}_{1, \tau}^{(j)}$ is the image of $\mathcal{F}_{0, \tau}^{(j)} \otimes_{k} k[\epsilon]$ for all $\tau$ and $j \leq j_{\tau}$ under the canonical isomorphism

$$
H_{\mathrm{dR}}^{1}\left(A_{1} / k[\epsilon]\right) \cong H_{\mathrm{cris}}^{1}\left(A_{0} / k[\epsilon]\right) \cong H_{\mathrm{dR}}^{1}\left(A_{0} / k\right) \otimes_{k} k[\epsilon] .
$$

We claim that the $f_{\mathfrak{p}}$-dimensional subspace $\oplus_{\tau \in \Sigma_{\mathfrak{p}, 0}} \mathrm{Fil}^{e_{\mathfrak{p}}-1} T_{x}(S)_{\tau}$ is contained in the kernel of $T_{x}(S) \rightarrow T_{y}(S)$. Indeed, if $\underline{A}_{1}$ is a lift corresponding to an element of this subspace and $\underline{A}_{1}^{\prime}$ is its image in $T_{y}(S)$ and $\alpha_{i}: A_{i} \rightarrow A_{i}^{\prime}$ are the specialisations of the universal isogeny $\alpha: A \rightarrow A^{\prime}$, then the commutativity of the diagram

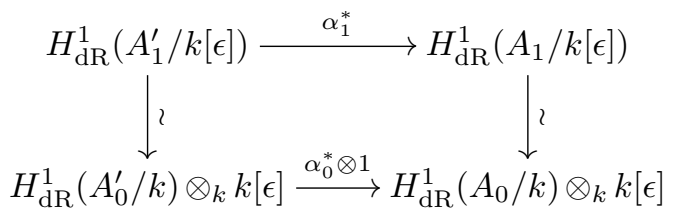

and the definition of $\widetilde{\Phi}_{\mathfrak{p}}$ imply that $\mathcal{F}_{1, \tau}^{\prime(j)}$ corresponds to $\mathcal{F}_{0, \tau}^{\prime(j)} \otimes_{k} k[\epsilon]$ for all $\tau$ and $j$. (Note in particular that $\mathcal{F}_{1, \tau}^{(1)}=\operatorname{ker}\left(\alpha_{1, \tau}^{*}\right)$ for all $\tau \in \Sigma_{\mathfrak{p}, 0}$ and that $H^{0}\left(A_{1}^{\prime}, \Omega_{A_{1}^{\prime} / k[\epsilon]}\right)_{\tau}$ corresponds to $H^{0}\left(A_{0}, \Omega_{A_{0}^{\prime} / k}\right)_{\tau} \otimes_{k} k[\epsilon]$ for all $\tau \in \Sigma_{0}$. ) It follows that $\underline{A}_{1}^{\prime}$ is the trivial deformation of $\underline{A}_{0}^{\prime}$, so the kernel of $T_{x}(S) \rightarrow T_{y}(S)$ has dimension $n \geq f_{\mathfrak{p}}$ as required.

Remark 6.1.2. The final part of the proof of the proposition shows that the kernel of the pullback map $\Phi_{\mathfrak{p}}^{*} \Omega_{\bar{Y}_{U} / \mathbb{F}}^{1} \rightarrow \Omega_{\bar{Y}_{U} / \mathbb{F}}^{1}$ is precisely

$$
\Phi_{\mathfrak{p}}^{*}\left(\oplus_{\tau \in \Sigma_{\mathfrak{p}, 0}} \operatorname{Fil}^{1}\left(\Omega_{\bar{Y}_{U} / \mathbb{F}}^{1}\right)_{\tau}\right) .
$$

Furthermore, a similar argument shows that the map preserves the Kodaira-Spencer decomposition and filtration, in the obvious sense, and induces isomorphisms

$$
\begin{array}{cc}
\Phi_{\mathfrak{p}}^{*}\left(\operatorname{Fil}^{j}\left(\Omega_{\bar{Y}_{U} / \mathbb{F}}^{1}\right)_{\tau} / \operatorname{Fil}^{1}\left(\Omega{\overline{Y_{U}}}_{\mathbb{F}}\right)_{\tau}\right) \stackrel{\sim}{\longrightarrow} \operatorname{Fil}^{j-1}\left(\Omega_{\bar{Y}_{U} / \mathbb{F}}^{1}\right)_{\tau}, & \text { if } \tau \in \Sigma_{\mathfrak{p}, 0}, j=1, \ldots, e_{\mathfrak{p}}, \\
\Phi_{\mathfrak{p}}^{*}\left(\operatorname{Fil}^{j}\left(\Omega_{\bar{Y}_{U} / \mathbb{F}}\right)_{\tau}\right) \stackrel{\sim}{\longrightarrow} \operatorname{Fil}^{j}\left(\Omega_{\bar{Y}_{U} / \mathbb{F}}^{1}\right)_{\tau}, & \text { if } \tau \notin \Sigma_{\mathfrak{p}, 0} .
\end{array}
$$




\subsection{Construction of $V_{\mathfrak{p}}$}

In this section we generalise the construction ${ }^{11}$ of $[12, \S 9.8]$ to define partial Frobenius operators, similar to the $V_{p}$-operator on classical modular forms.

We maintain the notation of Subsection 6.1 , so that $\widetilde{\Phi}_{\mathfrak{p}}$ is an endomorphism of $S=\widetilde{Y}_{U, \mathbb{F}}$ corresponding to the data $\left(A^{\prime}, \iota^{\prime}, \lambda^{\prime}, \eta^{\prime},\left(\mathcal{F}^{\prime}\right)^{\bullet}\right)$, where $A^{\prime}=A / H$ for a certain finite flat subgroup scheme $H \subset A[\mathfrak{p}]$, and $\alpha$ is the projection $A \rightarrow A^{\prime}$.

It is immediate from the definition of $\mathcal{F}_{\tau}^{\prime(j)}$ that $\alpha_{\tau}^{*}$ induces an isomorphism $\mathcal{L}_{\tau, j}^{\prime} \stackrel{\sim}{\longrightarrow} \mathcal{L}_{\tau, j}$ for all $j$ if $\tau \notin \Sigma_{\mathfrak{p}, 0}$, as well as

$$
\mathcal{L}_{\tau, j}^{\prime}=\mathcal{F}_{\tau}^{\prime(j)} / \mathcal{F}_{\tau}^{\prime(j-1)} \stackrel{\sim}{\longrightarrow} \mathcal{F}_{\tau}^{(j-1)} / \mathcal{F}_{\tau}^{(j-2)}=\mathcal{L}_{\tau, j-1}
$$

for $j=2, \ldots, e_{\mathfrak{p}}$ if $\tau \in \Sigma_{\mathfrak{p}, 0}$. To describe $\mathcal{L}_{\tau, 1}^{\prime}$ for $\tau \in \Sigma_{\mathfrak{p}, 0}$, note that since $H \subset \operatorname{ker}\left(\right.$ Frob $\left._{A}\right)$, there is an isogeny $\gamma: A^{\prime} \rightarrow \phi_{S}^{*} A$ such that $\gamma \circ \alpha=$ Frob $_{A}$. We thus obtain a surjection

$$
\gamma_{\tau}^{*}: \phi_{S}^{*}\left(\mathcal{F}_{\phi^{-1} \circ \tau}^{\left(e_{\mathfrak{p}}\right)}\right)=\operatorname{ker}\left(\operatorname{Frob}_{A}\right)_{\tau}^{*} \longrightarrow \operatorname{ker}\left(\alpha_{\tau}^{*}\right)=\mathcal{L}_{\tau, 1}^{\prime}
$$

Furthermore, $\phi_{S}^{*}\left(\mathcal{F}_{\phi^{-1} \circ \tau}^{\left(e_{\mathfrak{p}}-1\right)}\right)=\operatorname{ker}\left(\gamma_{\tau}^{*}\right)$, as can be seen on closed points, so we obtain a surjection, and hence an isomorphism, of line bundles

$$
\mathcal{L}_{\phi^{-1} \circ \tau, e_{\mathfrak{p}}}^{\otimes p} \cong \phi_{S}^{*}\left(\mathcal{L}_{\phi^{-1} \circ \tau, e_{\mathfrak{p}}}\right)=\phi_{S}^{*}\left(\mathcal{F}_{\phi^{-1} \circ \tau}^{\left(e_{\mathfrak{p}}\right)}\right) / \phi_{S}^{*}\left(\mathcal{F}_{\phi^{-1} \circ \tau}^{\left(e_{\mathfrak{p}}-1\right)}\right) \stackrel{\gamma_{\tau}^{*}}{\longrightarrow} \mathcal{L}_{\tau, 1}^{\prime}
$$

By construction, we have $\widetilde{\Phi}_{\mathfrak{p}}^{*} \mathcal{L}_{\tau, j}=\mathcal{L}_{\tau, j}^{\prime}$, so we have now defined isomorphisms

$$
\widetilde{\Phi}_{\mathfrak{p}}^{*} \mathcal{L}_{\theta} \cong \begin{cases}\mathcal{L}_{\sigma^{-1} \theta}^{\otimes n_{\theta}}, & \text { if } \theta \in \Sigma_{\mathfrak{p}} \\ \mathcal{L}_{\theta}, & \text { if } \theta \notin \Sigma_{\mathfrak{p}}\end{cases}
$$

Similarly, we find that if $\tau \in \Sigma_{\mathfrak{p}, 0}$, then $\gamma^{*}$ induces $\phi_{S}^{*}\left(\mathcal{P}_{\phi^{-1} \circ \tau, e_{\mathfrak{p}}}\right) \cong \mathcal{P}_{\tau, 1}^{\prime}$ and $\alpha^{*}$ induces $\mathcal{P}_{\tau, j}^{\prime} \cong \mathcal{P}_{\tau, j-1}$ for $j=2, \ldots, e_{\mathfrak{p}}$, so that $\widetilde{\Phi}_{\mathfrak{p}}^{*} \mathcal{N}_{\theta} \cong \mathcal{N}_{\sigma^{-1} \theta}^{\otimes n_{\theta}}$ if $\theta \in \Sigma_{\mathfrak{p}}$, while $\widetilde{\Phi}_{\mathfrak{p}}^{*} \mathcal{N}_{\theta} \cong \mathcal{N}_{\theta}$ if $\theta \notin \Sigma_{\mathfrak{p}}$. Taking tensor products, we thus obtain isomorphisms

$$
\Phi_{\mathfrak{p}}^{*} \widetilde{\mathcal{A}}_{\mathbf{k}, \mathbf{l}, \mathbb{F}} \cong \widetilde{\mathcal{A}}_{\mathbf{k}^{\prime \prime}, \mathbf{l}^{\prime \prime}, \mathbb{F}}
$$

for all $\mathbf{k}, \mathbf{l} \in \mathbb{Z}^{\Sigma}$, where

- $\quad k_{\theta}^{\prime \prime}=k_{\theta}$ and $l_{\theta}^{\prime \prime}=l_{\theta}$ if $\theta \notin \Sigma_{\mathfrak{p}}$ and

- $k_{\theta}^{\prime \prime}=n_{\sigma \theta} k_{\sigma \theta}$ and $l_{\theta}^{\prime \prime}=n_{\sigma \theta} l_{\sigma \theta}$ if $\theta \in \Sigma_{\mathfrak{p}}$.

Furthermore, it is straightforward to check that the isomorphisms are compatible with the descent data relative to $S=\widetilde{Y}_{U, \mathbb{F}} \rightarrow Y_{U, \mathbb{F}}$, so we obtain isomorphisms

$$
\Phi_{\mathfrak{p}}^{*} \mathcal{A}_{\mathbf{k}, \mathbf{l}, \mathbb{F}} \cong \mathcal{A}_{\mathbf{k}^{\prime \prime}, \mathbf{l}^{\prime \prime}, \mathbb{F}}
$$

for all $\mathbf{k}, \mathbf{l} \in \mathbb{Z}^{\Sigma}$ and sufficiently small $U$. Note that

$$
\mathbf{k}^{\prime \prime}=\mathbf{k}+\sum_{\theta \in \Sigma_{\mathfrak{p}}} k_{\theta} \mathbf{h}_{\theta} \quad \text { and } \quad \mathbf{l}^{\prime \prime}=\mathbf{l}+\sum_{\theta \in \Sigma_{\mathfrak{p}}} l_{\theta} \mathbf{h}_{\theta} .
$$

${ }^{11}$ The operators defined here differ slightly from the ones defined in [12] in the unramified case. The construction there is tailored to be compatible with the classical case and to be simply interpreted on $q$-expansions at cusps at $\infty$. Doing this in the general ramified case would introduce complications that make it seem not worthwhile. 
Remark 6.2.1. One can check that the resulting isomorphisms $\Phi_{\mathfrak{p}}^{*} \mathcal{A}_{2 \mathbf{e}_{\theta},-\mathbf{e}_{\theta}, \mathbb{F}} \cong$ $\mathcal{A}_{2 \mathbf{e}_{\theta},-\mathbf{e}_{\theta}, \mathbb{F}}\left(\right.$ for $\left.\theta \notin \Sigma_{\mathfrak{p}}\right)$ and

$$
\Phi_{\mathfrak{p}}^{*} \mathcal{A}_{2 \mathbf{e}_{\theta},-\mathbf{e}_{\tau}, \mathbb{F}} \cong \mathcal{A}_{2 \mathbf{e}_{\sigma^{-1} \theta_{\theta}},-\mathbf{e}_{\sigma-1_{\theta}}, \mathbb{F}}
$$

(for $\theta=\theta_{\mathfrak{p}, i, j}, j=2, \ldots, e_{\mathfrak{p}}$ ) are compatible via the Kodaira-Spencer isomorphisms of Theorem 3.3.1 with the corresponding isomorphisms

$$
\Phi_{\mathfrak{p}}^{*}\left(\operatorname{gr}^{j}\left(\Omega_{\bar{Y}_{U} / \mathbb{F}}^{1}\right)_{\tau}\right) \cong \operatorname{gr}^{j^{\prime}}\left(\Omega_{\bar{Y}_{U} / \mathbb{F}}^{1}\right)_{\tau}
$$

given by Remark 6.1.2, where $j^{\prime}=j$ if $\tau \notin \Sigma_{\mathfrak{p}, 0}$ and $j^{\prime}=j-1$ if $\tau \in \Sigma_{\mathfrak{p}, 0}$.

We are now ready to define the partial Frobenius operator (indexed by $\mathfrak{p}$ )

$$
V_{\mathfrak{p}}: M_{\mathbf{k}, \mathbf{l}}(U ; \mathbb{F}) \longrightarrow M_{\mathbf{k}^{\prime \prime}, \mathbf{l}^{\prime \prime}}(U, \mathbb{F})
$$

as the composite

$$
H^{0}\left(Y_{U, \mathbb{F}}, \mathcal{A}_{\mathbf{k}, \mathbf{l}, \mathbb{F}}\right) \stackrel{\Phi_{\mathfrak{p}}^{*}}{\longrightarrow} H^{0}\left(Y_{U, \mathbb{F}}, \Phi_{\mathfrak{p}}^{*} \mathcal{A}_{\mathbf{k}, \mathbf{l}, \mathbb{F}}\right) \stackrel{\sim}{\longrightarrow} H^{0}\left(Y_{U, \mathbb{F}}, \mathcal{A}_{\mathbf{k}^{\prime \prime}, \mathbf{l}^{\prime \prime}, \mathbb{F}}\right),
$$

where the second map is the isomorphism (13). It is immediate from the definition that $V_{\mathfrak{p}}$ is injective and that taking the direct sum over all weights yields an $\mathbb{F}$-algebra homomorphism

$$
\bigoplus_{\mathbf{k}, \mathbf{l} \in \mathbb{Z}^{\Sigma}} M_{\mathbf{k}, \mathbf{l}}(U ; \mathbb{F}) \longrightarrow \bigoplus_{\mathbf{k}, \mathbf{l} \in \mathbb{Z}^{\Sigma}} M_{\mathbf{k}, \mathbf{l}}(U ; \mathbb{F})
$$

for all sufficiently small $U$ containing $\mathrm{GL}_{2}\left(\mathcal{O}_{F, p}\right)$. It is also straightforward to check that $V_{\mathfrak{p}}$ is compatible with the Hecke action in the usual sense and hence defines a $\mathrm{GL}_{2}\left(\mathbb{A}_{F, \mathbf{f}}^{(p)}\right)$ equivariant map

$$
M_{\mathbf{k}, \mathbf{l}}(\mathbb{F}) \longrightarrow M_{\mathbf{k}^{\prime \prime}, \mathbf{l}^{\prime \prime}}(\mathbb{F}),
$$

where the spaces are defined in (6) as direct limits over sufficiently small $U$ containing $\mathrm{GL}_{2}\left(\mathcal{O}_{F, p}\right)$.

It will also be convenient at times to consider instead the operator

$$
V_{\mathfrak{p}}^{0}: M_{\mathbf{k}, \mathbf{l}}(U ; \mathbb{F}) \longrightarrow M_{\mathbf{k}^{\prime \prime}, \mathbf{l}}(U, \mathbb{F})
$$

defined by $V_{\mathfrak{p}}^{0}(f)=V_{\mathfrak{p}}(f) \prod_{\theta \in \Sigma_{\mathfrak{p}}} G_{\theta}^{-l_{\theta}}$, where $G_{\theta}$ is the trivialisation of $\mathcal{A}_{\mathbf{0}, \mathbf{h}_{\theta}, \mathbb{F}}$ defined at the end of Subsection 4.1. Thus, $V_{\mathfrak{p}}^{0}$ is also Hecke-equivariant but depends on the choice of uniformiser $\varpi_{\mathfrak{p}}$.

We also record the relation between the partial Frobenius operators and the $p$-power map. First note that the identification $\widetilde{\Phi}^{*}\left(\mathcal{F}_{\tau}^{(j)}\right)=\phi_{S}^{*}\left(\mathcal{F}_{\phi^{-1} \circ \tau}^{(j)}\right)$ arising from the definition of $\widetilde{\Phi}$ yields isomorphisms

$$
\widetilde{\Phi}^{*} \mathcal{L}_{\tau, j} \cong \phi_{S}^{*}\left(\mathcal{L}_{\phi^{-1} \circ \tau, j}\right) \cong \mathcal{L}_{\phi^{-1} \circ \tau, j}^{\otimes p}
$$

for all $\tau$ and $j$. We similarly have $\widetilde{\Phi}^{*} \mathcal{N}_{\tau, j} \cong \mathcal{N}_{\phi^{-1} \circ \tau, j}^{\otimes p}$, and taking tensor products and descending to $\bar{Y}$ yields isomorphisms $\Phi^{*} \mathcal{A}_{\mathbf{k}, \mathbf{l}, \mathbb{F}} \cong \mathcal{A}_{p \mathbf{k}^{\phi}, p \mathbf{l}^{\phi}, \mathbb{F}}$ for all $\mathbf{k}, \mathbf{l}$, where $k_{\theta_{\mathfrak{p}, i, j}}^{\phi}=k_{\theta_{\mathfrak{p}, i+1, j}}$ and hence an operator $V_{p}: M_{\mathbf{k}, \mathbf{l}}(U ; \mathbb{F}) \rightarrow M_{p \mathbf{k}^{\phi}, p \mathbf{l}^{\phi}}(U ; \mathbb{F})$. Similarly, the 
isomorphisms $\widetilde{\epsilon}^{*} \mathcal{F}_{\phi^{-1} \circ \tau}^{(j)} \cong \mathcal{F}_{\tau}^{(j)}$ yield $\widetilde{\epsilon}^{*} \mathcal{L}_{\phi^{-1} \circ \tau, j} \cong \mathcal{L}_{\tau, j}$ and $\widetilde{\epsilon}^{*} \mathcal{N}_{\phi^{-1} \circ \tau, j} \cong \mathcal{N}_{\tau, j}$ whose tensor products descend to isomorphisms $\epsilon^{*} \mathcal{A}_{\mathbf{k}^{\phi}, \mathbf{l}^{\phi}, \mathbb{F}} \stackrel{\sim}{\longrightarrow} \mathcal{A}_{\mathbf{k}, \mathbf{l}, \mathbb{F}}$, yielding a $\phi$-linear isomorphism $M_{\mathbf{k}^{\phi}, \mathbf{l}^{\phi}}(U ; \mathbb{F}) \rightarrow M_{\mathbf{k}, \mathbf{l}}(U ; \mathbb{F})$ which we denote $\epsilon_{\mathbf{k}, \mathbf{l}}$. Furthermore, the above isomorphisms of line bundles on $S$ are compatible in the sense that the resulting diagram

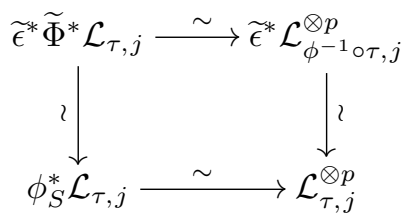

commutes, as does its analogue for the $\mathcal{N}_{\theta}$, from which it follows that the composite

$$
M_{\mathbf{k}, \mathbf{l}}(U ; \mathbb{F}) \stackrel{V_{p}}{\longrightarrow} M_{p \mathbf{k}^{\phi}, p \mathbf{l}^{\phi}}(U ; \mathbb{F}) \stackrel{\epsilon_{p \mathbf{k}, p \mathbf{1}}}{\longrightarrow} M_{p \mathbf{k}, p \mathbf{l}}(U ; \mathbb{F})
$$

is the $p$-power map.

Returning to the partial Frobenius operators, the isomorphisms between $\widetilde{\Phi}_{\mathfrak{p}}^{*} \mathcal{L}_{\theta}$ and $\mathcal{L}_{\sigma^{-1} \theta}^{\otimes n_{\theta}}$ (respectively $\mathcal{L}_{\theta}$ ) for $\theta \in \Sigma_{\mathfrak{p}}$ (respectively $\theta \notin \Sigma_{\mathfrak{p}}$ ) for different $\mathfrak{p} \in S_{p}$ are compatible with each other in the obvious sense and taken together with the formula $\prod_{\mathfrak{p} \in S_{p}} \widetilde{\Phi}_{\mathfrak{p}}^{e_{\mathfrak{p}}}=\nu \cdot \widetilde{\Phi}$ and the canonical isomorphism $\nu^{*} \mathcal{L}_{\theta} \cong \mathcal{L}_{\theta}$ yield the isomorphisms $\widetilde{\Phi}^{*} \mathcal{L}_{\tau, j} \cong \mathcal{L}_{\phi^{-1} \circ \tau, j}^{\otimes p}$ defined above. A similar assertion holds for the line bundles $\mathcal{N}_{\theta}$, and it follows that the operators $V_{\mathfrak{p}}$ for $\mathfrak{p} \in S_{p}$ commute with each other and that $\prod_{\mathfrak{p}} V_{\mathfrak{p}}^{e_{\mathfrak{p}}}=V_{p}$, so that

$$
\left(\epsilon_{p \mathbf{k}, p \mathbf{l}} \prod_{\mathfrak{p} \in S_{p}} V_{\mathfrak{p}}^{e_{\mathfrak{p}}}\right)(f)=f^{p} .
$$

\section{Compactifications and $q$-expansions}

\subsection{Toroidal compactifications}

We next recall how $q$-expansions of Hilbert modular forms are obtained using compactifications of Hilbert modular varieties. In this section we review properties of the toroidal compactification constructed by Rapoport [31] (see also [4] and [13]). We will consider toroidal compactifications only in the case $U=U(N)$, but we first describe the set of cusps adelically for any $U$ of level prime to $p$.

For an arbitrary open compact subgroup $U$ of $\mathrm{GL}_{2}\left(\mathbb{A}_{F, \mathbf{f}}\right)$ containing $\mathrm{GL}_{2}\left(\mathcal{O}_{F, p}\right)$, we define the set of cusps of $Y_{U}$ to be

$$
Y_{U}^{\infty}=B\left(\mathcal{O}_{F,(p)}\right)_{+} \backslash \mathrm{GL}_{2}\left(\mathbb{A}_{F, \mathbf{f}}^{(p)}\right) / U^{p}=B(F)_{+} \backslash \mathrm{GL}_{2}\left(\mathbb{A}_{F, \mathbf{f}}\right) / U,
$$

where $B$ denotes the subgroup of $\mathrm{GL}_{2}$ consisting of upper-triangular matrices. Similarly, we define the set of cusps of $\widetilde{Y}_{U}$ to be

$$
\tilde{Y}_{U}^{\infty}=B_{1}\left(\mathcal{O}_{F,(p)}\right)+\backslash \mathrm{GL}_{2}\left(\mathbb{A}_{F, \mathbf{f}}^{(p)}\right) / U^{p} .
$$

Note that the natural surjection $\tilde{Y}_{U}^{\infty} \rightarrow Y_{U}^{\infty}$ identifies $Y_{U}^{\infty}$ with the quotient of $\widetilde{Y}_{U}^{\infty}$ by the left action of $B\left(\mathcal{O}_{F,(p)}\right)_{+} / B_{1}\left(\mathcal{O}_{F,(p)}\right) \cong \mathcal{O}_{F,(p),+}^{\times}$. Furthermore, the subgroup $\left(\mathcal{O}_{F}^{\times} \cap U\right)^{2}$ 
acts trivially on $\tilde{Y}_{U}^{\infty}$, but the quotient $\mathcal{O}_{F,(p),+}^{\times} /\left(\mathcal{O}_{F}^{\times} \cap U\right)^{2}$ need not act freely; more precisely, the stabiliser of the cusp $B_{1}\left(\mathcal{O}_{F,(p)}\right) g U$ of $\widetilde{Y}_{U}$ is the $\operatorname{group} \operatorname{det}\left(g U g^{-1} \cap B(F)\right)_{+} \subset$ $\mathcal{O}_{F,+}^{\times}$, in which $\left(\mathcal{O}_{F}^{\times} \cap U\right)^{2}$ has finite index.

We also have a natural bijection between $Y_{U}^{\infty}$ and the set of isomorphism classes of data $(H, I,[\lambda],[\eta])$ where

- $H$ is a projective $\mathcal{O}_{F}$-module of rank 2 ;

- $I$ is an invertible submodule of $H$ such that $J:=H / I$ is invertible;

- $[\lambda]$ is a prime-to- $p$ orientation of $\wedge_{\mathcal{O}_{F}}^{2} H \cong I \otimes_{\mathcal{O}_{F}} J=I J$, by which we mean an $\mathcal{O}_{F,(p),+}^{\times}$-orbit of isomorphisms

$$
\lambda: \wedge_{\mathcal{O}_{F,(p)}}^{2} H_{(p)} \stackrel{\sim}{\longrightarrow} \mathcal{O}_{F,(p)} .
$$

- $[\eta]$ is a level $U^{p}$-structure on $H$; that is, a $U^{p}$-orbit of $\widehat{\mathcal{O}}_{F}^{(p)}$-linear isomorphisms

$$
\eta:\left(\widehat{\mathcal{O}}_{F}^{(p)}\right)^{2} \stackrel{\sim}{\longrightarrow} \widehat{\mathcal{O}}_{F}^{(p)} \otimes_{\mathcal{O}_{F}} H .
$$

The bijection is defined by associating the data $\left(H_{g}, I_{g},\left[\lambda_{g}\right],\left[\eta_{g}\right]\right)$ to the coset $B\left(\mathcal{O}_{F,(p)}\right)_{+} g U^{p}$, where $H_{g}=\widehat{\mathcal{O}}_{F}^{2} g^{-1} \cap F^{2}, I_{g}$ is its intersection with the subspace $\{0\} \times F$, $\lambda_{g}$ is induced by the determinant and $\eta_{g}$ is induced by right pre-multiplication by $g^{-1}$.

Note that to give a prime-to- $p$ orientation of $\wedge_{\mathcal{O}_{F}}^{2} H$ is equivalent to giving an $F_{+}^{\times}$orbit of isomorphisms $\wedge_{F}^{2}(\mathbb{Q} \otimes H) \stackrel{\sim}{\longrightarrow} F$, but the integrality condition is imposed for consistency with the fact that we have a bijection between $\widetilde{Y}_{U}^{\infty}$ and the set of isomorphism classes of data of the form $(H, I, \lambda,[\eta])$, which is similarly defined, $\mathcal{O}_{F,(p),+}^{\times}$-equivariant and compatible in the obvious sense with the bijection describing $Y_{U}^{\infty}$. In particular, if $U=U(1)=\mathrm{GL}_{2}\left(\widehat{\mathcal{O}}_{F}\right)$, then the map sending $(H, I,[\lambda],[\eta])$ to the pair $\left(\wedge_{\mathcal{O}_{F}}^{2} H, I\right)$ defines a bijection between $Y_{U}^{\infty}$ and $C_{F}^{+} \times C_{F}$, where $C_{F}^{(+)}$denotes the (strict) class group of $F$. For each such cusp we choose a polyhedral cone decomposition as in [31, Lemme 4.2] (with $U^{\prime}=U_{+}$in the notation there) for $\left(M^{*} \otimes \mathbb{R}\right)_{+} \cup\{0\}$, where

$$
\begin{aligned}
& M=\operatorname{Hom}_{\mathcal{O}_{F}}\left(I, \mathfrak{d}^{-1} J\right)=\mathfrak{d}^{-1} I^{-1} J, \\
& M^{*}=\operatorname{Hom}(M, \mathbb{Z}) \cong \operatorname{Hom}_{\mathcal{O}_{F}}(J, I)=J^{-1} I,
\end{aligned}
$$

and the positivity is induced by the orientation of $I \otimes_{\mathcal{O}_{F}} J$.

Suppose now that $U=U(N)$ for some $N \geq 3$ (not divisible by $p$ ) and that $\mathcal{O}$ contains the $N$ th roots of unity. The above choice of cone decomposition (for the image of each cusp of $\widetilde{Y}_{U}^{\infty}$ in $\left.Y_{U(1)}^{\infty}\right)$ yields a toroidal compactification ${ }^{12} \widetilde{Y}_{U} \hookrightarrow \widetilde{Y}_{U}^{\text {tor }}$ such that the set of (geometrically) connected components of its (reduced) closed subscheme $\widetilde{Z}_{U}^{\text {tor }}:=\widetilde{Y}_{U}^{\text {tor }}-\widetilde{Y}_{U}$ is identified with $\tilde{Y}_{U}^{\infty}$. The construction of $\tilde{Y}_{U}^{\text {tor }}$ identifies its completion along the component corresponding to a cusp $\widetilde{\mathcal{C}}$ represented by $(H, I, \lambda,[\eta])$ with the quotient

${ }^{12}$ Compactified in the sense that its (infinitely many) connected components are proper over $\mathcal{O}$. 
of a formal scheme ${ }^{13} \widehat{S}_{\widetilde{\mathcal{C}}}$ by an action of $V_{N}^{2}$, where $V_{N}=\operatorname{ker}\left(\mathcal{O}_{F}^{\times} \rightarrow\left(\mathcal{O}_{F} / N \mathcal{O}_{F}\right)^{\times}\right)$. Furthermore, this extends to an action of $\mathcal{O}_{F,+}^{\times}$on $\widehat{S}=\widehat{S}_{\widetilde{\mathcal{C}}}$, and we have an isomorphism

$$
\Gamma\left(\widehat{S}, \mathcal{O}_{\widehat{S}}\right) \cong \mathcal{O}\left[\left[q^{m}\right]\right]_{m \in N^{-1} M_{+} \cup\{0\}}
$$

compatible with the obvious action of $\mathcal{O}_{F,+}^{\times}$on the target. (The isomorphism depends on a choice of splitting of the exact sequence

$$
0 \rightarrow I \rightarrow H \rightarrow J \rightarrow 0
$$

of $\mathcal{O}_{F}$-modules; modifying the splitting by an element $\beta \in J^{-1} I \cong M^{*}$ alters it by composition with the automorphism defined by $q^{m} \mapsto \zeta_{N}^{-\beta(m)} q^{m}$ for $m \in N^{-1} M$.) We let $\xi: \widehat{S} \rightarrow \widetilde{Y}_{U}^{\text {tor }}$ denote the natural morphism of formal schemes, and we write $F_{\widehat{S}}$ for the field of fractions of $\Gamma\left(\widehat{S}, \mathcal{O}_{\widehat{S}}\right)$ and $\mu_{\nu}$ for the automorphism of $\widehat{S}$ defined by $\nu \in \mathcal{O}_{F,+}^{\times}$.

The construction of the toroidal compactification also extends the universal abelian scheme $A$ to a semi-abelian scheme $A^{\text {tor }}$ whose pullback to $\widehat{S}$ is identified with that of the Tate semi-abelian scheme ${ }^{14}$ associated to a quotient of the form

$$
T_{I, J}:=\left(\mathfrak{d}^{-1} I \otimes \mathbb{G}_{m}\right) / \widetilde{q}^{\mathfrak{d}^{-1} J},
$$

where $\widetilde{q}: \mathfrak{d}^{-1} J \rightarrow F_{\widehat{S}}^{\times} \otimes \mathfrak{d}^{-1} I$ is the homomorphism corresponding to the tautological element under the canonical isomorphism

$$
\operatorname{Hom}\left(M, F_{\widehat{S}}^{\times}\right)=\operatorname{Hom}_{\mathcal{O}_{F}}\left(\mathfrak{d}^{-1} J, \mathfrak{d}^{-1} I \otimes F_{\widehat{S}}^{\times}\right) .
$$

Similarly, its dual $A^{\vee}$ extends to a Tate semi-abelian scheme $\left(A^{\vee}\right)^{\text {tor }}$ whose pullback via $\xi$ is associated to $T_{\mathfrak{d} J^{-1}, \mathfrak{o} I^{-1}}$, with the isomorphism $\mathfrak{c d} \otimes_{\mathcal{O}_{F}} A^{\text {tor }} \rightarrow\left(A^{\vee}\right)^{\text {tor }}$ defined by the quasi-polarisation pulling back to the composite

$$
\mathfrak{c} \mathfrak{d} \otimes_{\mathcal{O}_{F}} T_{I, J} \stackrel{\sim}{\longrightarrow} \mathfrak{d}(I J)^{-1} \otimes_{\mathcal{O}_{F}} T_{I, J} \stackrel{\sim}{\longrightarrow} T_{\mathfrak{d} J^{-1}, \mathfrak{d} I^{-1}},
$$

where $\mathfrak{c}=\left\{\alpha \in F \mid \alpha \lambda(I J) \subset \mathcal{O}_{F}\right\}$, the first morphism is the isomorphism induced by $\lambda$ and the second is the canonical one.

The subschemes $Z_{\theta}$ of $\widetilde{Y}_{U}$ (defined in Subsection 4.2 by the vanishing of the partial Hasse invariants $H_{\theta}$ ) are closed in $\widetilde{Y}_{U}^{\text {tor }}$, and we let $\widetilde{Y}_{U}^{\text {ord }}$ (respectively $\widetilde{Y}_{U}^{\text {tord }}$ ) denote the complement of their union - that is, the ordinary locus, in $\widetilde{Y}_{U}$ (respectively $\tilde{Y}_{U}^{\text {tor }}$ ) - and we use similar notation for the restrictions of $A^{\text {tor }}$ and $\left(A^{\vee}\right)^{\text {tor }}$. Since the sheaf $\mathcal{L} i e\left(A^{\text {tord }} / \widetilde{Y}_{U}^{\text {tord }}\right)$ is locally free over $\mathcal{O}_{F} \otimes \mathcal{O}_{\widetilde{Y}_{U}^{\text {tord }}}$, the universal filtration $\mathcal{F}_{\tau}^{(j)}$ on

$$
\left(s_{*} \Omega_{A / \widetilde{Y}_{U}}^{1}\right)_{\tau} \cong \mathcal{H o m}_{\widetilde{\mathcal{Y}}_{U}}\left(\mathcal{L} i e\left(A / \widetilde{Y}_{U}\right)_{\tau}, \mathcal{O}_{\widetilde{Y}_{U}}\right)
$$

${ }^{13}$ The formal scheme depends on the chosen cone decomposition $\left\{\sigma_{\alpha}^{\widetilde{\mathcal{C}}}\right\}$ and is denoted $S_{N}\left(\left\{\sigma_{\alpha}^{\widetilde{\mathcal{C}}}\right\}\right)^{\wedge}$ in $[4$, Subsection 3.4.2].

${ }^{14}$ More precisely, the formal scheme $\widehat{S}$ has an open cover by affine formal subschemes $\operatorname{Spf} R_{\sigma}$ (indexed by cones $\sigma$ ) such that $\operatorname{Spec} R_{\sigma} \times_{\widetilde{Y}_{U}^{\text {tor }}} A^{\text {tor }}$ is identified with the semi-abelian scheme $T_{I, J}$ over $\operatorname{Spec} R_{\sigma}$. The compatibilities in the discussion that follows are then systematically checked by verifying them over the open subschemes $\operatorname{Spec} R_{\sigma}^{0}=\operatorname{Spec} R_{\sigma} \times_{\widetilde{Y}_{U}^{\text {tor }}} \widetilde{Y}_{U}$. 
extends canonically to one on $\mathcal{H} o m_{\mathcal{O}_{\tilde{Y}_{U}}^{\text {tor }}}\left(\mathcal{L} i e\left(A^{\text {tor }} / \widetilde{Y}_{U}^{\text {tor }}\right)_{\tau}, \mathcal{O}_{\widetilde{Y}_{U}^{\text {tor }}}\right)$ for each $\tau \in \Sigma_{0}$. Furthermore, its pullback to $\widehat{S}$ is identified (in the notation of (1)) with

$$
0 \subset t_{\tau, 1}\left(I^{-1} \otimes \mathcal{O}_{\widehat{S}}\right)_{\tau} \subset t_{\tau, 2}\left(I^{-1} \otimes \mathcal{O}_{\widehat{S}}\right) \subset \cdots \subset t_{\tau, e_{\mathfrak{p}}}\left(I^{-1} \otimes \mathcal{O}_{\widehat{S}}\right)_{\tau}=\left(I^{-1} \otimes \mathcal{O}_{\widehat{S}}\right)_{\tau}
$$

under the canonical isomorphism

$$
\mathcal{H o m}_{\mathcal{O}_{\widehat{S}}}\left(\mathcal{L} i e\left(T_{I, J} / \widehat{S}\right), \mathcal{O}_{\widehat{S}}\right) \cong \operatorname{Hom}\left(\mathfrak{d}^{-1} I, \mathcal{O}_{\widehat{S}}\right) \cong I^{-1} \otimes \mathcal{O}_{\widehat{S}}
$$

We thus obtain extensions $\widetilde{\mathcal{L}}_{\theta}^{\text {tor }}$ of the line bundles $\widetilde{\mathcal{L}}_{\theta}=\widetilde{\mathcal{L}}_{\mathfrak{p}, i, j}$ to $\widetilde{Y}_{U}^{\text {tor }}$ whose pullback to $\widehat{S}$ is identified with $\left(I^{-1}\right)_{\theta} \otimes_{\mathcal{O}} \mathcal{O}_{\widehat{S}}$, where $\left(I^{-1}\right)_{\theta}$ is defined by $(2)$.

Similarly, $\operatorname{Lie}\left(\left(A^{\vee}\right)^{\text {tord }} / \widetilde{Y}_{U}^{\text {tord }}\right)$ is locally free over $\mathcal{O}_{F} \otimes \mathcal{O}_{\widetilde{Y}_{U}^{\text {tord }}}$, but the line bundles $\widetilde{\mathcal{M}}_{\theta}=\widetilde{\mathcal{M}}_{\mathfrak{p}, i, j}$ over $\widetilde{Y}_{U}^{\text {ord }}$ are canonically identified with

$$
\left(R^{1} s_{*} \mathcal{O}_{A^{\text {ord }}}\right)_{\tau}\left[u-\theta\left(\varpi_{\mathfrak{p}}\right)\right] \cong \mathcal{L} i e\left(\left(A^{\vee}\right)^{\text {ord }} / \widetilde{Y}_{U}^{\text {ord }}\right)_{\tau}\left[u-\theta\left(\varpi_{\mathfrak{p}}\right)\right]
$$

It follows that each $\widetilde{\mathcal{M}}_{\theta}$ extends to a line bundle $\widetilde{\mathcal{M}}_{\theta}^{\text {tor }}$ on $\widetilde{Y}_{U}^{\text {tor }}$ such that the identification

$$
\mathcal{L} i e\left(T_{\mathfrak{d} J^{-1}, \mathfrak{d} I^{-1}} / \widehat{S}\right)=J^{-1} \otimes \mathcal{O}_{\widehat{S}}
$$

induces an isomorphism

$$
\xi^{*} \widetilde{\mathcal{M}}_{\theta}^{\text {tor }} \cong\left(J^{-1} \otimes \mathcal{O}_{\widehat{S}}\right)_{\tau}\left[u-\theta\left(\varpi_{\mathfrak{p}}\right)\right] \cong\left(\mathfrak{d} J^{-1} \otimes \mathcal{O}_{\widehat{S}}\right)_{\tau} \otimes_{\mathcal{O}[u], \theta} \mathcal{O}
$$

We can thus identify the pullback $\xi^{*} \widetilde{\mathcal{N}}_{\theta}^{\text {tor }}$ of the line bundle $\widetilde{\mathcal{N}}_{\theta}^{\text {tor }}=\widetilde{\mathcal{L}}_{\theta}^{\text {tor }} \otimes_{\mathcal{O}_{\tilde{Y}_{U}}^{\text {tor }}} \widetilde{\mathcal{M}}_{\theta}^{\text {tor }}$ with $\left(\mathfrak{d}(I J)^{-1}\right)_{\theta} \otimes_{\mathcal{O}} \mathcal{O}_{\widehat{S}}$, which the polarisation in turn identifies with $(\mathfrak{c} \mathfrak{d})_{\theta} \otimes_{\mathcal{O}} \mathcal{O}_{\widehat{S}}$ in the notation of (2). Finally, it follows that the automorphic bundles $\widetilde{\mathcal{A}}_{\mathbf{k}, \mathbf{l}}$ extend to line bundles $\widetilde{\mathcal{A}}_{\mathbf{k}, \mathbf{l}}^{\text {tor }}$ on $\widetilde{Y}_{U}^{\text {tor }}$ such that

$$
\xi^{*} \widetilde{\mathcal{A}}_{\mathbf{k}, \mathbf{l}}^{\text {tor }} \cong D_{\mathbf{k}, \mathbf{l}} \otimes_{\mathcal{O}} \mathcal{O}_{\widehat{S}}, \quad \text { where } D_{\mathbf{k}, \mathbf{l}}:=\bigotimes_{\theta \in \Sigma}\left(\left(I^{-1}\right)_{\theta}^{\otimes k_{\theta}} \otimes\left(\mathfrak{d}(I J)^{-1}\right)_{\theta}^{\otimes l_{\theta}}\right)
$$

(the tensor products being over $\mathcal{O}$ ). We refer to this isomorphism as the canonical trivialisation of $\xi^{*} \widetilde{\mathcal{A}}_{\mathbf{k}, 1}^{\text {tor }}$.

Next we consider the completion of $\tilde{Y}_{U}^{\text {tor }}$ along the component corresponding to the cusp $\widetilde{\mathcal{C}}$ represented by $(H, I, \lambda,[\eta])$, which we denote $\left(\widetilde{Y}_{U^{\text {tor }}}\right) \widetilde{\mathcal{C}}_{\widetilde{\mathcal{C}}}$. We now describe the global sections of the completions of the line bundles $\widetilde{\mathcal{A}}_{\mathbf{k}, 1}^{\text {tor }}$ using the identification $\left(\widetilde{Y}_{U}^{\text {tor }}\right)_{\widetilde{\mathcal{C}}}^{\wedge}=$ $\widehat{S} / V_{N}^{2}$ and taking invariants under the action of $V_{N}^{2}$ on their trivialisations over $\widehat{S}$. Note firstly that $\Gamma\left(\left(\widetilde{Y}_{U}^{\text {tor }}\right)_{\widetilde{\mathcal{C}}}^{\wedge}, \mathcal{O}_{\left(\widetilde{Y}_{U}^{\text {tor }}\right)_{\widetilde{\mathcal{C}}}}\right)=\Gamma\left(\widehat{S}, \mathcal{O}_{\widehat{S}}\right)^{V_{N}^{2}}$ corresponds to

$$
\left\{\sum r_{m} q^{m} \in \mathcal{O}\left[\left[q^{m}\right]\right]_{m \in N^{-1} M_{+} \cup\{0\}} \mid r_{\alpha^{2} m}=r_{m} \forall \alpha \in V_{N}, m \in N^{-1} M_{+}\right\}
$$

under the isomorphism of (18). One then finds that the descent data for $\xi^{*} A^{\text {tor }}$ is provided by the isomorphisms $T_{I, J} \stackrel{\sim}{\longrightarrow} \mu_{\alpha^{2}}^{*} T_{I, J}$ induced by $\alpha \otimes 1$ on $\mathfrak{d}^{-1} I \otimes \mathbb{G}_{m}$, from 
which it follows that the descent data for $\xi^{*} \widetilde{\mathcal{L}}_{\theta}^{\text {tor }}$ is provided on the trivialisation by the isomorphisms

$$
\left(I^{-1}\right)_{\theta} \otimes_{\mathcal{O}} \mu_{\alpha^{2}}^{*} \mathcal{O}_{\widehat{S}} \stackrel{\sim}{\longrightarrow}\left(I^{-1}\right)_{\theta} \otimes_{\mathcal{O}} \mathcal{O}_{\widehat{S}}
$$

induced by $\theta(\alpha)$ on $\left(I^{-1}\right)_{\theta}$. On the other hand, the descent data for $\xi^{*} \widetilde{\mathcal{M}}_{\theta}^{\text {tor }}$ is similarly induced on the canonical trivialisation by $\theta(\alpha)^{-1}$, so that the resulting trivialisation of $\xi^{*} \widetilde{\mathcal{N}}_{\theta}^{\text {tor }}$ descends to $\left(\widetilde{Y}_{U^{\text {tor }}}\right)_{\widetilde{\mathcal{C}}}^{\wedge}$ (in fact extending the one already defined over $\widetilde{Y}_{U}$ via the choice of generator $t_{\tau, j}\left(f^{\prime}\left(\varpi_{\mathfrak{p}}\right) \otimes 1\right)$ of $\left.(\mathfrak{c} \mathfrak{d})_{\theta}\right)$. Since $\Gamma\left(\left(\widetilde{Y}_{U}^{\text {tor }}\right)_{\widetilde{\mathcal{C}}},\left(\widetilde{\mathcal{A}}_{\mathbf{k}, 1}^{\text {tor }}\right)_{\widetilde{\mathcal{C}}}^{\wedge}\right)=\Gamma\left(\widehat{S}, \xi^{*} \widetilde{\mathcal{A}}_{\mathbf{k}, 1}^{\text {tor }}\right)^{V_{N}^{2}}$, we conclude the following.

Proposition 7.1.1. Suppose that $U=U(N)$ and $\mathcal{O}$ contains the Nth roots of unity. Then the isomorphism (18) and the canonical trivialisation (20) identify $\Gamma\left(\left(\widetilde{Y}_{U}^{\text {tor }}\right)_{\widetilde{\mathcal{C}}},\left(\widetilde{\mathcal{A}}_{\mathbf{k}, 1}^{\text {tor }}\right)_{\widetilde{\mathcal{C}}}^{\wedge}\right)$ with

$$
\left\{\sum_{m \in N^{-1} M_{+} \cup\{0\}} b \otimes r_{m} q^{m} \mid r_{\alpha^{2} m}=\chi_{\mathbf{k}}(\alpha) r_{m} \forall \alpha \in V_{N}, m \in N^{-1} M_{+}\right\},
$$

where $b$ is any choice of basis for $D_{\mathbf{k}, \mathbf{l}}$.

\subsection{Minimal compactifications}

We now recall the construction due to Chai [4] of minimal compactifications of Hilbert modular varieties. The presentation in [4] is very concise with numerous typos, but a more detailed treatment of the construction can be found in [13] in the case of $U_{1}(\mathfrak{n})$ (with different conventions than ours) and of the descriptions of $q$-expansions in that case in [5].

We continue to assume for the moment that $U=U(N)$ for some sufficiently large $N$ prime to $p$. The minimal compactification $\widetilde{Y}_{U} \hookrightarrow \widetilde{Y}_{U}^{\text {min }}$ is then constructed as in [4, §4] or $[13, \S 8]$. More precisely, letting $\mathbf{t}=\sum \mathbf{e}_{\theta}$ and taking the global sections of $\oplus_{k \geq 0} \widetilde{\mathcal{A}}_{k \mathbf{t}, \mathbf{0}}^{\text {tor }}$ over each component of $\tilde{Y}_{U}^{\text {tor }}$ yields a projective scheme over $\mathcal{O}$ containing the corresponding component of the Deligne-Pappas model as an open subscheme. Gluing their ordinary loci to $\widetilde{Y}_{U}$ along $\widetilde{Y}_{U}^{\text {ord }}$ yields a scheme $\widetilde{Y}_{U}^{\text {min }}$ and a proper morphism $\pi: \widetilde{Y}_{U}^{\text {tor }} \rightarrow \widetilde{Y}_{U}^{\text {min }}$ such that $\widetilde{\imath}: \widetilde{Y}_{U} \rightarrow \widetilde{Y}_{U}^{\text {min }}$ is an open immersion. Furthermore, the (reduced) complement $\widetilde{Y}_{U}^{\min }-\widetilde{Y}_{U}$ is an infinite disjoint union of copies of $\operatorname{Spec} \mathcal{O}$ indexed by $\widetilde{Y}_{U}^{\infty}$, the pre-image of each in $\widetilde{Y}_{U}^{\text {tor }}$ being the corresponding connected component of $\widetilde{Z}_{U}^{\text {tor }}$, and the scheme $\tilde{Y}_{U}^{\text {min }}$ is independent of the choice of cone decomposition in the construction of $\tilde{Y}_{U}^{\text {tor }}$.

Now recall that the Koecher principle implies that $\mathcal{O}_{\widetilde{Y}_{U}^{\min }}=\pi_{*} \mathcal{O}_{\widetilde{Y}_{U}^{\text {tor }}}=\widetilde{\iota}_{*} \mathcal{O}_{\widetilde{Y}_{U}}$, so that $\mathcal{O}_{\widetilde{Y}_{U}^{\text {min }}, \widetilde{\mathcal{C}}}^{\wedge}=\Gamma\left(\left(\widetilde{Y}_{U}^{\text {tor }}\right)_{\widetilde{\mathcal{C}}}^{\wedge}, \mathcal{O}_{\left(\widetilde{Y}_{U}^{\text {tor }}\right)_{\widetilde{\mathcal{C}}}}\right)$ is the ring described by $(21)$, where we have written $\widetilde{\mathcal{C}}$ for the corresponding point of $\widetilde{Y}_{U}^{\mathrm{min}}$. Furthermore, the argument of [31, Prop. 4.9] shows that $\widetilde{\iota}_{*} \widetilde{\mathcal{A}}_{\mathbf{k}, \mathbf{l}}=\pi_{*} \widetilde{\mathcal{A}}_{\mathbf{k}, \mathbf{l}}^{\text {tor }}$ (see the discussion following [31, Def. 6.10], or view $\widetilde{Y}_{U}$ as a disjoint union of PEL Shimura varieties and apply [28, Thm. 2.5]), so the theorem on formal functions gives that $\left.\left(\iota_{*} \widetilde{\mathcal{A}}_{\mathbf{k}, 1}\right) \underset{\widetilde{\mathcal{C}}}{\wedge}=\Gamma\left(\left(\widetilde{Y}_{U^{\text {tor }}}\right)_{\widetilde{\mathcal{C}}}^{\wedge},\left(\widetilde{\mathcal{A}}_{\mathbf{k}, 1}^{\text {tor }}\right)_{\widetilde{\mathcal{C}}}^{\wedge}\right)\right)$ is the $\mathcal{O}_{\widetilde{Y}_{U}^{\text {min }}, \widetilde{\mathcal{C}}}^{\wedge}$-module described in Proposition 7.1.1. (Note that $\widetilde{\iota}_{*} \widetilde{\mathcal{A}}_{\mathbf{k}, \mathbf{l}}$ is coherent but not necessarily invertible.) 
Similarly, for any $\mathcal{O}$-algebra $R$, we may identify $\left(\widetilde{\iota}_{R, *} \widetilde{\mathcal{A}}_{\mathbf{k}, \mathbf{l}, R}\right)_{\widetilde{\mathcal{C}}}^{\wedge}$ with

$$
\left\{\sum_{m \in N^{-1} M_{+} \cup\{0\}} b \otimes r_{m} q^{m} \mid r_{\alpha^{2} m}=\chi_{\mathbf{k}, R}(\alpha) r_{m} \forall \alpha \in V_{N}, m \in N^{-1} M_{+}\right\}
$$

as a module over $\mathcal{O}_{\widetilde{Y}_{U, R}^{\min }, \widetilde{\mathcal{C}}}^{\wedge}$, which the Koecher principle and (18) identify with

$$
\left\{\sum r_{m} q^{m} \in R\left[\left[q^{m}\right]\right]_{m \in N^{-1} M_{+} \cup\{0\}} \mid r_{\alpha^{2} m}=r_{m} \forall \alpha \in V_{N}, m \in N^{-1} M_{+}\right\},
$$

where $\widetilde{\iota}_{R}: \widetilde{Y}_{U, R} \rightarrow \widetilde{Y}_{U, R}^{\min }$ is the base change of $\widetilde{\iota}$ to $R$, the completions are at the fibre over $\widetilde{\mathcal{C}}$ and $b$ is any basis for $D_{\mathbf{k}, 1}$.

The compatibility of the choices of polyhedral cone decompositions ensures that the natural action of $\mathcal{O}_{F,(p),+}$ on $\widetilde{Y}_{U}$ extends (uniquely) to one on $\tilde{Y}_{U}^{\text {tor }}$. Furthermore, the stabiliser of each component of $\widetilde{Z}_{U}^{\text {tor }}$ is $V_{N,+}$, and the action of $V_{N,+}$ on each completion $\left(\widetilde{Y}_{U^{\text {tor }}}\right) \stackrel{\widehat{\mathcal{C}}}{=} \widehat{S} / V_{N}^{2}$ is induced by an action of $V_{N,+}$ on $\widehat{S}$ such that the effect of $\nu \in V_{N,+}$ on global sections of $\mathcal{O}_{\widehat{S}}$ is induced by multiplication by $\nu^{-1}$ on $M$. We see also that the canonical isomorphism $A \rightarrow \nu^{*} A$ extends to an isomorphism $A^{\text {tor }} \rightarrow \nu^{*} A^{\text {tor }}$ whose pullback via $\xi$ is induced by the identity on $\mathfrak{d}^{-1} I \otimes \mathbb{G}_{m}$, from which it follows that the action of $\nu$ is compatible with the canonical trivialisation of the line bundle $\widetilde{\mathcal{L}}_{\theta}^{\text {tor }}$ over $\widehat{S}$. On the other hand, the induced isomorphisms $\nu^{*} \widetilde{\mathcal{M}}_{\theta}^{\text {tor }} \rightarrow \mathcal{M}_{\theta}^{\text {tor }}$ and $\nu^{*} \widetilde{\mathcal{N}}_{\theta}^{\text {tor }} \rightarrow \widetilde{\mathcal{N}}_{\theta}^{\text {tor }}$ pull back to ones corresponding to multiplication by $\theta(\nu)$.

Since $\widetilde{Y}_{U}^{\mathrm{min}}$ is a disjoint union of projective schemes over $\mathcal{O}$ on which $\mathcal{O}_{F,(p),+}^{\times} / V_{N}^{2}$ acts with finite stabilisers, the quotient scheme exists, and we define this to be the minimal compactification $Y_{U}^{\mathrm{min}}$ of $Y_{U}$. We thus obtain an open immersion $\iota: Y_{U} \rightarrow Y_{U}^{\mathrm{min}}$ such that $Y_{U}^{\min }$ is projective over $\mathcal{O}$ and the (reduced) complement of $Y_{U}$ is a disjoint union of copies of $\operatorname{Spec} \mathcal{O}$ in canonical bijection with the set of cusps $Y_{U}^{\infty}$. Furthermore, we again have that $\iota_{*} \mathcal{O}_{Y_{U}}=\mathcal{O}_{Y_{U}^{\min }}$, and its completion $\mathcal{O}_{Y_{U}^{\min }, \mathcal{C}}^{\wedge}$ at the cusp $\mathcal{C}$ represented by $(H, I,[\lambda],[\eta])$ is identified under $(21)$ with

$$
\left\{\sum r_{m} q^{m} \in \mathcal{O}\left[\left[q^{m}\right]\right]_{m \in N^{-1} M_{+} \cup\{0\}} \mid r_{\nu m}=r_{m} \forall \nu \in V_{N,+}, m \in N^{-1} M_{+}\right\}
$$

(where the identification depends as in (18) on a choice of splitting of the exact sequence $0 \rightarrow I \rightarrow H \rightarrow J \rightarrow 0)$. Now suppose that $R$ is an $\mathcal{O}$-algebra such that $\chi_{\mathbf{k}+2 \mathbf{l}, R}$ is trivial on $V_{N}$, so that the line bundle $\widetilde{\mathcal{A}}_{\mathbf{k}, \mathbf{l}, R}$ descends to one over $Y_{U, R}$ which we denote by $\mathcal{A}_{\mathbf{k}, \mathbf{l}, R}$. We then see that $\iota_{*} \mathcal{A}_{\mathbf{k}, \mathbf{l}, R}$ is a coherent sheaf on $Y_{U, R}^{\min }$ whose completion at the (base change to $R$ of the) cusp $\mathcal{C}$ is identified under (22) with

$$
\left\{\sum_{m \in N^{-1} M_{+} \cup\{0\}} b \otimes r_{m} q^{m} \mid r_{\nu^{-1} m}=\chi_{\mathbf{l}, R}(\nu) r_{m} \forall \nu \in V_{N,+}, m \in N^{-1} M_{+}\right\} .
$$

In particular, $\iota_{*} \mathcal{A}_{\mathbf{k}, \mathbf{l}, R}$ is a line bundle if $\chi_{\mathbf{l}, R}$ is trivial on $V_{N,+}$.

Suppose now that $U^{\prime}$ is any sufficiently small open compact subgroup of $\mathrm{GL}_{2}\left(\widehat{\mathcal{O}}_{F}\right)$ containing $\mathrm{GL}_{2}\left(\mathcal{O}_{F, p}\right)$. One can then carry out a construction similar to the one above 
to obtain the minimal compactification or choose an $N$ prime to $p$ such that $U(N) \subset U^{\prime}$, extend the natural (right) action of $U^{\prime} / U(N)$ on $Y_{U(N)}$ to $Y_{U(N)}^{\min }$ and take the quotient; we do the latter (see [13] for the former in the case of $U^{\prime}=U_{1}(\mathfrak{n})$ ). Firstly, our choice of polyhedral cone decompositions ensures that the natural right action of $U^{\prime} / U$ on $\widetilde{Y}_{U}$ extends to $\tilde{Y}_{U}^{\text {tor }}$, where $U=U(N)$ for some choice of $N$ as above. Denoting the resulting automorphism of $\widetilde{Y}_{U}^{\text {tor }}$ by $\widetilde{\rho}_{g}$ for $g \in U^{\prime}$, the canonical identification of the universal $A$ over $\widetilde{Y}_{U}$ with its pullback extends to an identification $A^{\text {tor }}=\widetilde{\rho}_{g}^{*} A^{\text {tor }}$, giving rise to canonical isomorphisms $\widetilde{\rho}_{g}^{*} \widetilde{\mathcal{A}}_{k \mathbf{t}, \mathbf{0}}^{\text {tor }}=\widetilde{\mathcal{A}}_{k \mathbf{t}, \mathbf{0}}^{\text {tor }}$, and hence to an action of $U^{\prime} / U$ on $\widetilde{Y}_{U}^{\text {min }}$ extending its action on $\tilde{Y}_{U}$. Moreover, the action commutes with the natural action of $\mathcal{O}_{F,(p),+}$, so it descends to an action on $Y_{U}^{\mathrm{min}}$ extending the action on $Y_{U}$. We denote the resulting automorphisms of $Y_{U}^{\mathrm{min}}$ by $\rho_{g}$ and define $Y_{U^{\prime}}^{\min }$ to be the quotient of $Y_{U}^{\mathrm{min}}$ by the action of $U^{\prime} / U$ (which we will soon see is independent of the choice of $N$ in its definition).

Identifying the set of components of $Y_{U}^{\mathrm{min}}-Y_{U}$ with $Y_{U}^{\infty}$, the resulting action of $g \in U^{\prime}$ is given by pre-composing $\eta$ with right multiplication by $g^{-1}$, so the set of components of $Y_{U^{\prime}}^{\min }-Y_{U^{\prime}}$ may be identified with $Y_{U^{\prime}}^{\infty}$. For each cusp $\mathcal{C}^{\prime} \in Y_{U^{\prime}}^{\infty}$, the completion $\mathcal{O}_{Y_{U^{\prime}}^{\min }, \mathcal{C}^{\prime}}^{\wedge}$ is identified with the subring of $\mathcal{O}_{Y_{U}^{\text {min }}, \mathcal{C}}^{\wedge}$ invariant under the stabiliser of $\mathcal{C}$ in $U^{\prime} / U$, where $\mathcal{C}$ is any cusp of $Y_{U}$ in the pre-image of $\mathcal{C}^{\prime}$. Choose such an $(H, I,[\lambda],[\eta])$ representing $\mathcal{C}$ and a splitting $\sigma: H \stackrel{\sim}{\longrightarrow} J \times I$ and let

$$
\Gamma_{\mathcal{C}}=\left\{\left(\begin{array}{cc}
\alpha & \beta \\
0 & \delta
\end{array}\right) \mid \alpha \in \mathcal{O}_{F}^{\times}, \beta \in J^{-1} I, \delta \in \alpha \mathcal{O}_{F,+}^{\times}\right\},
$$

which we view as acting on $J \times I$ by right multiplication. The stabiliser of $\mathcal{C}$ is then the set of classes $U g=g U \in U^{\prime} / U$ such that

$$
g \equiv \eta^{-1} \sigma^{-1} \gamma \sigma \eta \bmod N \widehat{\mathcal{O}}_{F} \text { for some } \gamma \in \Gamma_{\mathcal{C}},
$$

and we let $\Gamma_{\mathcal{C}, U^{\prime}}=\Gamma_{\mathcal{C}} \cap \sigma \eta U^{\prime} \eta^{-1} \sigma^{-1}$. Thus, the stabiliser of $\mathcal{C}$ is the image of the homomorphism $s: \Gamma_{\mathcal{C}, U^{\prime}} \longrightarrow U^{\prime} / U$ defined by $\gamma \mapsto \eta^{-1} \sigma^{-1} \gamma \sigma \eta U$.

We claim that if $g=s(\gamma)$, then $\rho_{g}^{*}$ on $\mathcal{O}_{Y_{U}^{\min }, \mathcal{C}}^{\wedge}=H^{0}\left(\widehat{S}, \mathcal{O}_{\widehat{S}}\right)^{V_{N},+}$ is induced by an automorphism $\psi_{\gamma}$ of $\widehat{S}$ whose effect on global sections corresponds to the map defined by

$$
\psi_{\gamma}^{*}: q^{m} \mapsto \zeta_{N}^{-\beta\left(\alpha^{-1} N m\right)} q^{\alpha^{-1} \delta m}
$$

under (18) and the identification $M^{*}=J^{-1} I$ of (17). Indeed, letting $\nu$ denote $\alpha \delta$ (as well as the automorphism of $\widetilde{Y}_{U}^{\text {tor }}$ defined by its effect on the universal polarisation), we see that $\delta \otimes 1$ on $T_{I, J}$ defines an isomorphism $\xi^{*} \rho_{g}^{*} \nu^{*} A^{\text {tor }} \stackrel{\sim}{\longrightarrow} \psi_{\gamma}^{*} \xi^{*} A^{\text {tor }}$ compatible with all auxiliary data, from which one deduces that $\nu \circ \rho_{g} \circ \xi=\xi \circ \psi_{\gamma}$. Note also that (25) defines an action of $\Gamma_{\mathcal{C}}$ on $\Gamma\left(\widehat{S}, \mathcal{O}_{\widehat{S}}\right)$ which factors through the surjection

$$
\left.\begin{array}{ccc}
\Gamma_{\mathcal{C}} & \longrightarrow & \left(J^{-1} I \otimes \mathbb{Z} / N \mathbb{Z}\right) \rtimes \mathcal{O}_{F,+}^{\times} \\
0 & \beta \\
0 & \delta
\end{array}\right) \mapsto \begin{gathered}
\left(-\alpha^{-1} \beta, \alpha^{-1} \delta\right),
\end{gathered}
$$

and the latter group acts on $\Gamma\left(\widehat{S}, \mathcal{O}_{\widehat{S}}\right)^{V_{N},+}$ via its quotient

$$
\left(J^{-1} I \otimes \mathbb{Z} / N \mathbb{Z}\right) \rtimes\left(\mathcal{O}_{F,+}^{\times} / V_{N,+}\right) .
$$


We conclude that $\mathcal{O}_{Y_{U^{\prime}}^{\min }, \mathcal{C}^{\prime}}^{\wedge}=H^{0}\left(\widehat{S}, \mathcal{O}_{\widehat{S}}\right)^{\Gamma_{\mathcal{C}, U^{\prime}}}$ is identified with

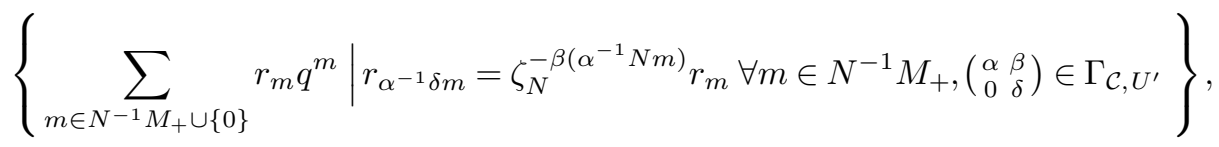

where we recall that the isomorphism may depend on the choice of the splitting $\sigma$ and that we view $\beta$ as an element of $M^{*}$. We note in particular that if $U^{\prime}=U\left(N^{\prime}\right)$ for some $N^{\prime} \mid N$, then the resulting description of $\mathcal{O}_{Y_{U^{\prime}}^{\min }, \mathcal{C}^{\prime}}^{\wedge}$ coincides with the one previously obtained, from which it follows that the same holds for the scheme $Y_{U^{\prime}}^{\min }$ and hence that $Y_{U^{\prime}}^{\min }$ is independent of the choice of $N$ in its definition (for any sufficiently small $U^{\prime}$ containing $\mathrm{GL}_{2}\left(\mathcal{O}_{F, p}\right)$.)

Suppose now that $\mathbf{k}, \mathbf{l} \in \mathbb{Z}^{\Sigma}$ and $R$ is an $\mathcal{O}$-algebra such that $\chi_{\mathbf{k}+2 \mathbf{l}, R}$ is trivial on $U^{\prime} \cap \mathcal{O}_{F}^{\times}$and consider the automorphic bundle $\mathcal{A}_{\mathbf{k}, \mathbf{l}, R}^{\prime}$ on $Y_{U^{\prime}, R}$. Letting $\iota_{R}^{\prime}$ denote the open immersion of $Y_{U^{\prime}, R}$ in $Y_{U^{\prime}, R}^{\min }$, similar considerations to those above show that $\iota_{R, *}^{\prime} \mathcal{A}_{\mathbf{k}, \mathbf{l}, R}^{\prime}$ is a coherent sheaf on $Y_{U, R}^{\min }$ whose completion at $\mathcal{C}^{\prime}$ is identified with the $\mathcal{O}_{Y_{U^{\prime}, R}^{\min }, \mathcal{C}^{-}}^{\wedge}$-module

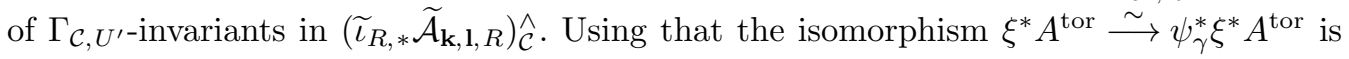
induced by $\delta \otimes 1$ on the Tate semi-abelian scheme, we find that the resulting automorphism multiplies the canonical trivialisation $(20)$ of $\xi^{*} \widetilde{\mathcal{A}}_{\mathbf{k}, \mathbf{l}, R}^{\text {tor }}$ by $\chi_{\mathbf{l}, R}(\alpha) \chi_{\mathbf{k}+\mathbf{l}, R}(\delta)$. We therefore conclude the following.

Proposition 7.2.1. If $\chi_{\mathbf{k}+21, R}$ is trivial on $U^{\prime} \cap \mathcal{O}_{F}^{\times}$, then $\iota_{R, *}^{\prime} \mathcal{A}_{\mathbf{k}, \mathbf{l}, R}^{\prime}$ is a coherent sheaf on $Y_{U^{\prime}, R}^{\min }$ whose completion at (the fibre over) $\mathcal{C}^{\prime}$ is identified by the Koecher principle and Proposition \%.1.1 with

$$
\left\{\begin{array}{c|c}
\sum_{m \in N^{-1} M_{+} \cup\{0\}} b \otimes r_{m} q^{m} & \begin{array}{c}
r_{\alpha^{-1} \delta m}=\zeta_{N}^{-\beta\left(\alpha^{-1} N m\right)} \chi_{\mathbf{l}, R}(\alpha) \chi_{\mathbf{k}+\mathbf{l}, R}(\delta) r_{m} \\
\text { for all } m \in N^{-1} M_{+},\left(\begin{array}{cc}
\alpha & \beta \\
0 & \delta
\end{array}\right) \in \Gamma_{\mathcal{C}, U^{\prime}}
\end{array}
\end{array}\right\} .
$$

Note that the description of $\mathcal{O}_{Y_{U^{\prime}, R}^{\min }, \mathcal{C}^{\prime}}^{\wedge}=\left(\iota_{R, *}^{\prime} \mathcal{O}_{Y_{U^{\prime}, R}^{\min }}\right) \hat{\mathcal{C}}^{\prime}$ may be viewed as a special case (with $\mathbf{k}=\mathbf{l}=\mathbf{0}$ ), as can the prior formula for $U=U(N)$. Furthermore, the identifications are compatible in the obvious senses with base changes $R \rightarrow R^{\prime}$, inclusions $U^{\prime \prime} \subset U^{\prime}$ (provided the splittings $\sigma$ are chosen compatibly) and the natural algebra structure on $\bigoplus_{\mathbf{k}, \mathbf{l}} \mathcal{A}_{\mathbf{k}, \mathbf{l}, R}^{\prime}$ (taking the direct sum over $\mathbf{k}, \mathbf{l}$ as in the statement).

Recall that the $q$-expansion principle allows one to characterise Hilbert modular forms in terms of their $q$-expansions.

Proposition 7.2.2. If $C \subset Y_{U^{\prime}}^{\infty}$ is any set of cusps containing at least one on each component of $Y_{U^{\prime}}$, then the natural map

$$
M_{\mathbf{k}, \mathbf{l}}\left(U^{\prime} ; R\right)=H^{0}\left(Y_{U^{\prime}}^{\min }, \iota_{R, *}^{\prime} \mathcal{A}_{\mathbf{k}, \mathbf{l}, R}^{\prime}\right) \longrightarrow \bigoplus_{\mathcal{C}^{\prime} \in C}\left(\iota_{R, *}^{\prime} \mathcal{A}_{\mathbf{k}, \mathbf{l}, R}^{\prime}\right)_{\mathcal{C}^{\prime}}^{\wedge}
$$

is injective. 
Note also that we may replace $D_{\mathbf{k}, \mathbf{l}} \otimes_{\mathcal{O}}$. with $D_{\mathbf{k}, \mathbf{l}, R} \otimes_{R}$. in the description of $q$ expansions over $R$. In particular, if $R$ is an $\mathbb{F}$-algebra, the identification

$$
\begin{aligned}
\left(I^{-1}\right)_{\theta} \otimes_{\mathcal{O}} \mathbb{F} & =\left(I^{-1} \otimes \mathcal{O}\right)_{\tau} \otimes_{\mathcal{O}[u], \theta} \mathbb{F}=t_{\tau, j}\left(I_{\mathfrak{p}}^{-1} \otimes_{W\left(\mathcal{O}_{F} / \mathfrak{p}\right), \tau} \mathcal{O}\right) \otimes_{\mathcal{O}[u], \theta} \mathbb{F} \\
& =t_{\tau, j}\left(I_{\mathfrak{p}}^{-1} \otimes_{W\left(\mathcal{O}_{F} / \mathfrak{p}\right), \tau} \mathcal{O}\right) /\left(u, \mathfrak{m}_{\mathcal{O}}\right) t_{\tau, j}\left(I_{\mathfrak{p}}^{-1} \otimes_{W\left(\mathcal{O}_{F} / \mathfrak{p}\right), \tau} \mathcal{O}\right) \\
& =u^{e_{\mathfrak{p}}-j}\left(I_{\mathfrak{p}}^{-1} \otimes_{W\left(\mathcal{O}_{F} / \mathfrak{p}\right), \tau} \mathbb{F}\right) / u^{e_{\mathfrak{p}}-j+1}\left(I_{\mathfrak{p}}^{-1} \otimes_{W\left(\mathcal{O}_{F} / \mathfrak{p}\right), \tau} \mathbb{F}\right)
\end{aligned}
$$

yields a canonical isomorphism

$$
\left(I^{-1}\right)_{\theta} \otimes_{\mathcal{O}} R=\left(\mathfrak{p}^{e_{\mathfrak{p}}-j} I^{-1} / \mathfrak{p}^{e_{\mathfrak{p}}-j+1} I^{-1}\right) \otimes_{\mathcal{O}_{F} / \mathfrak{p}, \tau} R
$$

The analogous formula holds for the factors $\left(\mathfrak{d}(I J)^{-1}\right)_{\theta}$ appearing in the definition of $D_{\mathbf{k}, \mathbf{l}}$.

The condition on the $q$-expansion coefficients in the description of the completions in Proposition 7.2.1 simplifies for certain standard level structures and cusps, as in [12, Prop. 9.1.2]. Suppose that $\mathfrak{n}$ is an ideal of $\mathcal{O}_{F}$ such that $\chi_{\mathbf{k}+2 \mathbf{l}, R}$ is trivial on $V_{\mathfrak{n}}=\operatorname{ker}\left(\mathcal{O}_{F}^{\times} \rightarrow\right.$ $\left.\left(\mathcal{O}_{F} / \mathfrak{n}\right)^{\times}\right)$. Letting $U^{\prime}=U(\mathfrak{n})$, we have

$$
\Gamma_{\mathcal{C}, U^{\prime}}=\left\{\left(\begin{array}{cc}
\alpha & \beta \\
0 & \delta
\end{array}\right) \mid \alpha \in V_{\mathfrak{n}}, \beta \in \mathfrak{n} M^{*}, \delta \in \alpha V_{\mathfrak{n},+}\right\}
$$

for every $\operatorname{cusp} \mathcal{C}$ of $Y_{U}$. Note that $m \in \mathfrak{n}^{-1} M$ if and only if $\beta(N m) \in N \mathbb{Z}$ for all $\beta \in \mathfrak{n} M^{*}$ and that $\alpha, \delta \in V_{\mathfrak{n}}$ implies that $\chi_{\mathbf{l}, R}(\alpha) \chi_{\mathbf{k}+2 \mathbf{l}}(\delta)=\chi_{\mathbf{l}, R}\left(\alpha \delta^{-1}\right)$, so we see that

$$
\left(\iota_{R, *}^{\prime} \mathcal{A}_{\mathbf{k}, \mathbf{l}, R}^{\prime}\right)_{\mathcal{C}^{\prime}} \simeq\left\{\sum_{m \in\left(\mathfrak{n}^{-1} M\right)_{+} \cup\{0\}} b \otimes r_{m} q^{m} \mid r_{\nu^{-1} m}=\chi_{\mathbf{l}, R}(\nu) r_{m} \text { for all } \nu \in V_{\mathfrak{n},+}\right\}
$$

for every cusp $\mathcal{C}^{\prime}$ of $Y_{U^{\prime}}$.

Keep the same assumption on $\mathfrak{n}$ but now let $U^{\prime}=U_{1}(\mathfrak{n})$ and suppose that $\mathcal{C}^{\prime}$ is a cusp of $Y_{U^{\prime}}$ 'at $\infty$ ' in the sense that $\eta(0,1) \in I+\mathfrak{n} \widehat{H}^{(p)}$. We then find that

$$
\Gamma_{\mathcal{C}, U^{\prime}}=\left\{\left(\begin{array}{cc}
\alpha & \beta \\
0 & \delta
\end{array}\right) \mid \alpha \in \delta \mathcal{O}_{F,+}^{\times}, \beta \in M^{*}, \delta \in V_{\mathfrak{n}}\right\},
$$

and we similarly conclude that

$$
\left(\iota_{R, *}^{\prime} \mathcal{A}_{\mathbf{k}, \mathbf{l}, R}^{\prime}\right)_{\mathcal{C}^{\prime}}^{\wedge} \simeq\left\{\sum_{m \in M_{+} \cup\{0\}} b \otimes r_{m} q^{m} \mid r_{\nu^{-1} m}=\chi_{\mathbf{l}, R}(\nu) r_{m} \text { for all } \nu \in \mathcal{O}_{F,+}\right\} .
$$

We remark that every component of $Y_{U^{\prime}}$ contains such cusps (in the obvious sense) and that in this case the isomorphism is independent of the choice of splitting $\sigma$.

\subsection{Kodaira-Spencer filtration}

We next explain how the Kodaira-Spencer filtration on differentials extends to compactifications. 
We maintain the notation from the preceding section. In particular, we first assume $U=U(N)$ for some $N$ prime to $p$ before deducing results for more general level structures. The construction of $\widetilde{Y}_{U}^{\text {tor }}$ via torus embeddings then yields a canonical isomorphism

$$
\xi^{*}\left(\Omega_{\widetilde{Y}_{U}^{\text {tor }} / \mathcal{O}}^{1}\left(\log \widetilde{Z}_{U}^{\text {tor }}\right)\right) \cong N^{-1} M \otimes \mathcal{O}_{\widehat{S}}
$$

for each cusp $\widetilde{\mathcal{C}}$ of $\widetilde{Y}_{U}$ under which the descent data relative to the quotient map $\widehat{S}=$ $\widehat{S}_{\widetilde{\mathcal{C}}} \rightarrow\left(\widetilde{Y}_{U}^{\text {tor }}\right)_{\widetilde{\mathcal{C}}}^{\wedge}$ corresponds to that induced by the obvious action of $V_{N}^{2}$ on $N^{-1} M$ and the completion of the canonical derivation

$$
d: \mathcal{O}_{\widetilde{Y}_{U}^{\text {tor }}} \rightarrow \Omega_{\widetilde{Y}_{U}^{\text {tor }} / \mathcal{O}}^{1}\left(\log \widetilde{Z}_{U}^{\text {tor }}\right)
$$

pulls back to a derivation $\mathcal{O}_{\widehat{S}} \rightarrow N^{-1} M \otimes \mathcal{O}_{\widehat{S}}$ whose effect on global sections corresponds under (18) to the map defined by

$$
\sum_{m \in N^{-1} M_{+} \cup\{0\}} r_{m} q^{m} \mapsto \sum_{m \in N^{-1} M_{+} \cup\{0\}} m \otimes r_{m} q^{m} .
$$

Recall also that

$$
\begin{aligned}
s_{*} \Omega_{A^{\text {ord }} / \widetilde{Y}_{U}^{\text {ord }}}^{1} & \cong \mathcal{H o m} \mathcal{O}_{\tilde{Y}_{U}^{\text {ord }}}\left(\mathcal{L} i e\left(A^{\text {ord }} / \widetilde{Y}_{U}^{\text {ord }}\right), \mathcal{O}_{\widetilde{Y}_{U}^{\text {ord }}}\right) \\
\text { and } R^{1} s_{*} \mathcal{O}_{A^{\text {ord }}} & \cong \mathcal{L} i e\left(\left(A^{\text {ord }}\right)^{\vee} / \widetilde{Y}_{U}^{\text {ord }}\right)
\end{aligned}
$$

are locally free sheaves of $\mathcal{O}_{F} \otimes \mathcal{O}_{\widetilde{Y}_{U}^{\text {ord }}}$-modules over $\tilde{Y}_{U}^{\text {ord }}$, and therefore so is

$$
s_{*} \Omega_{A^{\text {ord }} / \widetilde{Y}_{U}^{\text {ord }}}^{\text {ord }} \mathcal{O}_{F} \otimes \mathcal{O}_{\tilde{Y}_{U}^{\text {ord }}} s_{*} \Omega_{\left(A^{\text {ord }}\right)^{\vee} / \widetilde{Y}_{U}^{\text {ord }}}^{1} .
$$

Decomposing this sheaf over embeddings $\tau \in \Sigma_{0}$ and equipping it with the filtration defined by the images of the endomorphisms $t_{\tau, j}$ defined by (1), we see that the successive quotients

$$
\begin{aligned}
& t_{j}\left(s_{*} \Omega_{A^{\text {ord }} / \widetilde{Y}_{U}^{\text {ord }}}^{1} \otimes_{\mathcal{O}_{F} \otimes \mathcal{O}_{\tilde{Y}_{U}^{\text {ord }}}} s_{*} \Omega_{\left(A^{\text {ord }}\right)^{\vee} / \widetilde{Y}_{U}^{\text {ord }}}^{1}\right)_{\tau} \otimes_{\mathcal{O}[u], \theta} \mathcal{O} \\
& \cong \mathcal{H o m}_{\mathcal{O}_{S}}\left(R^{1} s_{*} \mathcal{O}_{A^{\text {ord }}}[u-\theta(\varpi)], t_{j}\left(s_{*} \Omega_{\left(A^{\text {ord }}\right)^{\vee} / \widetilde{Y}_{U}^{\text {ord }}}\right)_{\tau} \otimes_{\mathcal{O}[u], \theta} \mathcal{O}\right)
\end{aligned}
$$

(where $\tau=\tau_{i}, t_{j}=t_{\tau, j}$ and $\theta=\theta_{\mathfrak{p}, i, j}$ ) are canonically identified with the automorphic bundles $\widetilde{\mathcal{A}}_{2 \mathbf{e}_{\theta},-\mathbf{e}_{\theta}}$ over $\tilde{Y}_{U}^{\text {ord }}$. Furthermore, the proof of Theorem 3.3.1 shows that the natural map

$$
s_{*} \Omega_{A^{\text {ord }} / \widetilde{Y}_{U}^{\text {ord }}}^{1} \otimes \mathcal{O}_{F} \otimes \mathcal{O}_{\tilde{Y}_{U}^{\text {ord }}} s_{*} \Omega_{\left(A^{\text {ord }}\right)^{\vee} / \widetilde{Y}_{U}^{\text {ord }}}^{1} \longrightarrow \Omega_{\widetilde{Y}_{U}^{\text {ord }} / \mathcal{O}}^{1}
$$

arising from Grothendieck-Messing theory or, equivalently, the Gauss-Manin connection on $\mathcal{H}_{\mathrm{dR}}^{1}\left(A^{\text {ord }} / \widetilde{Y}_{U}^{\text {ord }}\right)$ (see $\left.[27, \S 2.1 .7]\right)$ is an isomorphism. In particular, the KodairaSpencer filtration on $\Omega_{\widetilde{Y}_{U}^{\text {ord }} / \mathcal{O}}^{1}$ corresponds under (28) to the one defined by the images of the endomorphisms $t_{\tau, j}$. Furthermore, $(28)$ extends over $\widetilde{Y}_{U}^{\text {tord }}$ to an isomorphism

$$
\begin{gathered}
\left.\mathcal{H o m}_{\mathcal{O}_{\tilde{Y}_{U}^{\text {tord }}}\left(\mathfrak{d} \otimes \mathcal{O}_{F}\right.} \mathcal{L} i e\left(A^{\text {tord }} / \widetilde{Y}_{U}^{\text {tord }}\right) \otimes_{\mathcal{O}_{F} \otimes \mathcal{O}_{\tilde{Y}_{U}^{\text {tord }}}} \mathcal{L} i e\left(\left(A^{\text {tord }}\right)^{\vee} / \widetilde{Y}_{U}^{\text {tord }}\right), \mathcal{O}_{\widetilde{Y}_{U}^{\text {tord }}}\right) \\
\stackrel{\sim}{\longrightarrow} \Omega_{\widetilde{Y}_{U}^{\text {tord }} / \mathcal{O}}^{1}\left(\log \widetilde{Z}_{U}^{\text {tor }}\right)
\end{gathered}
$$


whose pullback via $\xi=\xi_{\widetilde{\mathcal{C}}}$ for each cusp $\widetilde{\mathcal{C}}$ of $\widetilde{Y}_{U}$ is compatible with the canonical isomorphisms of the pullback of each with $M \otimes \mathcal{O}_{\widehat{S}}=N^{-1} M \otimes \mathcal{O}_{\widehat{S}}$ (the latter via (27)). Indeed, the existence of the extension and the claimed compatibility follow from the analogous well-known result after base change to $\mathbb{C}$. We therefore conclude that the Kodaira-Spencer filtration on $\Omega_{\widetilde{Y}_{U} / \mathcal{O}}^{1}$ extends over $\widetilde{Y}_{U}^{\text {tor }}$ in the form of a decomposition

$$
\Omega_{\widetilde{Y}_{U}^{\text {tor }} / \mathcal{O}}^{1}\left(\log \widetilde{Z}_{U}^{\text {tor }}\right)=\bigoplus_{\mathfrak{p} \in S_{p}} \bigoplus_{i \in \mathbb{Z} / f_{\mathfrak{p}} \mathbb{Z}}\left(\Omega_{\widetilde{Y}_{U}^{\text {tor }} / \mathcal{O}}^{1}\left(\log \widetilde{Z}_{U}^{\text {tor }}\right)\right)_{\mathfrak{p}, i},
$$

together with an increasing filtration of length $e_{\mathfrak{p}}$ on each component $\left(\Omega_{\widetilde{Y}_{U}^{\text {tor }} / \mathcal{O}}^{1}\left(\log \widetilde{Z}_{U}^{\text {tor }}\right)\right)_{\mathfrak{p}, i}$ and isomorphisms

$$
\widetilde{\mathcal{A}}_{2 \mathbf{e}_{\theta},-\mathbf{e}_{\theta}}^{\text {tor }} \stackrel{\sim}{\longrightarrow} \operatorname{gr}^{j}\left(\Omega_{\widetilde{Y}_{U}^{\text {tor }} / \mathcal{O}}^{1}\left(\log \widetilde{Z}_{U}^{\text {tor }}\right)\right)_{\mathfrak{p}, i}
$$

Furthermore, for each cusp $\widetilde{\mathcal{C}}$ of $\widetilde{Y}_{U}$ and embeddings $\tau=\tau_{\mathfrak{p}, i}$ and $\theta=\theta_{\mathfrak{p}, i, j}$, the pullback via $\xi=\xi_{\widetilde{\mathcal{C}}}$ of $\mathrm{Fil}^{j}\left(\Omega_{\widetilde{Y}_{U}^{\text {tor }} / \mathcal{O}}^{1}\left(\log \widetilde{Z}_{U}^{\text {tor }}\right)\right)_{\mathfrak{p}, i}$ corresponds to $t_{j}\left(N^{-1} M \otimes \mathcal{O}\right)_{\tau} \otimes_{\mathcal{O}} \mathcal{O}_{\widehat{S}}=t_{j}\left(\mathfrak{d}^{-1} I^{-1} J \otimes\right.$ $\mathcal{O})_{\tau} \otimes_{\mathcal{O}} \mathcal{O}_{\widehat{S}}$ under $(27)$, and the resulting isomorphism

$$
\xi^{*} \widetilde{\mathcal{A}}_{2 \mathbf{e}_{\theta},-\mathbf{e}_{\theta}}^{\text {tor }} \stackrel{\sim}{\longrightarrow} \operatorname{gr}^{j}\left(\xi^{*}\left(\Omega_{\widetilde{Y}_{U}^{\text {tor }} / \mathcal{O}}^{1}\left(\log \widetilde{Z}_{U}^{\text {tor }}\right)\right)_{\mathfrak{p}, i}\right) \cong\left(\mathfrak{d}^{-1} I^{-1} J \otimes \mathcal{O}\right)_{\theta} \otimes_{\mathcal{O}} \mathcal{O}_{\widehat{S}}
$$

coincides with the canonical trivialisation of (20).

We now interpret this in the context of minimal compactifications. First we note that the argument of [31, Prop. 4.9] yields a Koecher principle for $\Omega_{\widetilde{Y}_{U}^{\text {tor }} / \mathcal{O}}^{1}\left(\log \widetilde{Z}_{U}^{\text {tor }}\right)$, so that

$$
\pi_{*}\left(\Omega_{\widetilde{Y}_{U}^{\text {tor }} / \mathcal{O}}^{1}\left(\log \widetilde{Z}_{U}^{\text {tor }}\right)\right) \longrightarrow \widetilde{\iota}_{*}\left(\Omega_{\widetilde{Y}_{U} / \mathcal{O}}^{1}\right)
$$

is an isomorphism of coherent sheaves on $\widetilde{Y}_{U}^{\text {min }}$ whose completion at the cusp $\widetilde{\mathcal{C}}$ is identified with $\left(M \otimes \mathcal{O}\left[\left[q^{m}\right]\right]_{m \in N^{-1} M_{+} \cup\{0\}}\right)^{V_{N}^{2}}$

$$
=\left\{\sum_{m \in N^{-1} M_{+}} c_{m} \otimes q^{m} \mid c_{\nu m}=\nu c_{m} \forall \nu \in V_{N}^{2}, m \in N^{-1} M_{+}\right\} .
$$

(Note that $c_{0} \in M^{V_{N}^{2}}=0$.) Furthermore, the completion at $\widetilde{\mathcal{C}}$ of the canonical derivation $\mathcal{O}_{\widetilde{Y}_{U}^{\min }} \rightarrow \widetilde{\iota}_{*} \Omega_{\widetilde{Y}_{U} / \mathcal{O}}^{1}$ is given by $\sum r_{m} q^{m} \mapsto \sum m \otimes r_{m} q^{m}$.

We see also from the description of the extension of the Kodaira-Spencer filtration to $\Omega_{\widetilde{Y}_{U}^{\text {tor }} / \mathcal{O}}^{1}\left(\log \widetilde{Z}_{U}^{\text {tor }}\right)$ in terms of $q$-expansions that

$$
\pi_{*}\left(\operatorname{Fil}^{j}\left(\Omega_{\widetilde{Y}_{U}^{\text {tor }} / \mathcal{O}}^{1}\left(\log \widetilde{Z}_{U}^{\text {tor }}\right)\right)_{\mathfrak{p}, i}\right)=\widetilde{\iota}_{*}\left(\mathrm{Fil}^{j}\left(\Omega_{\widetilde{Y}_{U} / \mathcal{O}}^{1}\right)_{\mathfrak{p}, i}\right),
$$

with completion at $\widetilde{\mathcal{C}}$ given by $\left(t_{j}(M \otimes \mathcal{O})_{\tau} \otimes_{\mathcal{O}} \mathcal{O}\left[\left[q^{m}\right]\right]_{m \in N^{-1} M_{+} \cup\{0\}}\right)^{V_{N}^{2}}$

$$
=\left\{\sum_{m \in N^{-1} M_{+}} c_{m} \otimes q^{m} \mid c_{\nu m}=(\nu \otimes 1) c_{m} \forall \nu \in V_{N}^{2}, m \in N^{-1} M_{+}\right\} .
$$


Furthermore, since $V_{N}^{2}$ acts freely on $N^{-1} M_{+}\left(\right.$and $\left.M_{\theta}^{V_{N}^{2}}=0\right)$, the morphisms

$$
\widetilde{\iota}_{*}\left(\operatorname{Fil}^{j}\left(\Omega_{\widetilde{Y}_{U} / \mathcal{O}}^{1}\right)_{\mathfrak{p}, i}\right) \rightarrow \widetilde{\iota}_{*} \widetilde{\mathcal{A}}_{2 \mathbf{e}_{\theta},-\mathbf{e}_{\theta}}
$$

are surjective on completions at cusps and hence surjective. It follows that the graded pieces of (the obvious extension of) the Kodaira-Spencer filtration on $\widetilde{\iota}_{*} \Omega_{\widetilde{Y}_{U} / \mathcal{O}}^{1}$ are canonically isomorphic to $\widetilde{\iota}_{*} \widetilde{\mathcal{A}}_{2 \mathbf{e}_{\theta},-\mathbf{e}_{\theta}}$.

The constructions above are compatible with the natural actions of $\mathcal{O}_{F,(p),+}^{\times}$on $\tilde{Y}_{U}^{\text {tor }}$, $\widetilde{Y}_{U}^{\mathrm{min}}$ and $M$, so that the resulting descriptions carry over to $Y_{U}^{\mathrm{min}}$. More precisely, $\iota_{*}\left(\Omega_{Y_{U} / \mathcal{O}}^{1}\right)$ is a coherent sheaf on $Y_{U}^{\mathrm{min}}$ whose completion at the cusp $\mathcal{C}$ is identified with $\left(M \otimes \mathcal{O}\left[\left[q^{m}\right]\right]_{m \in N^{-1} M_{+} \cup\{0\}}\right)^{V_{N,+}}$

$$
=\left\{\sum_{m \in N^{-1} M_{+}} c_{m} \otimes q^{m} \mid c_{\nu m}=\nu c_{m} \forall \nu \in V_{N,+}, m \in N^{-1} M_{+}\right\},
$$

in terms of which the canonical derivation is $\sum r_{m} q^{m} \mapsto \sum m \otimes r_{m} q^{m}$. Furthermore, the completion at $\mathcal{C}$ of $\operatorname{Fil}^{j}\left(\iota_{*}\left(\Omega_{Y_{U} / \mathcal{O}}^{1}\right)_{\mathfrak{p}, i}\right):=\iota_{*}\left(\mathrm{Fil}^{j}\left(\Omega_{Y_{U} / \mathcal{O}}^{1}\right)_{\mathfrak{p}, i}\right)$ is identified with $\left(t_{j}(M \otimes \mathcal{O})_{\tau} \otimes_{\mathcal{O}} \mathcal{O}\left[\left[q^{m}\right]\right]_{m \in N^{-1} M_{+} \cup\{0\}}\right)^{V_{N,+}}$, and the natural maps

$$
\operatorname{gr}^{j}\left(\iota_{*}\left(\Omega_{Y_{U} / \mathcal{O}}^{1}\right)_{\mathfrak{p}, i}\right) \hookrightarrow \iota_{*}\left(\operatorname{gr}^{j}\left(\Omega_{Y_{U} / \mathcal{O}}^{1}\right)_{\mathfrak{p}, i}\right) \stackrel{\sim}{\longrightarrow} \iota_{*} \mathcal{A}_{2 \mathbf{e}_{\theta},-\mathbf{e}_{\theta}}
$$

are isomorphisms whose completions at the cusps are induced by the surjections $t_{j}(M \otimes$ $\mathcal{O})_{\tau} \rightarrow M_{\theta}$.

Suppose now that $U^{\prime}$ is an arbitrary sufficiently small open compact subgroup of $\mathrm{GL}_{2}\left(\mathbb{A}_{F, \mathbf{f}}\right)$ of level prime to $p$, and choose $N$ so that $U(N) \subset U^{\prime}$. The constructions above are then also compatible with the natural actions of $U^{\prime}$, so we arrive at similar conclusions with minor modifications to the descriptions of completions that result from taking invariants under $\Gamma_{\mathcal{C}, U^{\prime}}$. We omit the details, but we remark that letting $L$ (respectively $V$ ) denote the kernel (respectively image) of the homomorphism

$$
\begin{aligned}
& \Gamma_{\mathcal{C}, U^{\prime}} /\left(\mathcal{O}_{F}^{\times} \cap U^{\prime}\right) \quad \rightarrow \quad \mathcal{O}_{F,+}^{\times} \\
& \left(\begin{array}{cc}
\alpha & \beta \\
0 & \delta
\end{array}\right) \cdot\left(\mathcal{O}_{F}^{\times} \cap U^{\prime}\right) \mapsto \alpha^{-1} \delta,
\end{aligned}
$$

the coefficients $r_{m}$ of $q$-expansions in $\mathcal{O}_{Y_{U^{\prime}}^{\min }, \mathcal{C}^{\prime}}^{\wedge}$ are indexed by $m \in\left(L^{*}\right)_{+} \cup\{0\}$ (where $L$ is identified with a finite index subgroup of $M$ ). Since $V$ acts freely on $L^{*}$ (twisting $q$-expansion coefficients by a possibly nontrivial cocycle valued in $L \otimes \mu_{N}(\mathcal{O})$ ), we still obtain the isomorphism of (29) with $U$ replaced by $U^{\prime}$, identifying the graded pieces of the Kodaira-Spencer filtration on $\iota_{*} \Omega_{Y_{U^{\prime}} / \mathcal{O}}^{1}$ with the sheaves $\iota_{*} \mathcal{A}_{2 \mathbf{e}_{\theta},-\mathbf{e}_{\theta}}$.

The description of the extension of the Kodaira-Spencer filtration over compactifications also applies after base change to an arbitrary $\mathcal{O}$-algebra $R$, with one significant difference. If $R$ is not flat over $\mathcal{O}$, then the modules $M \otimes R$ (and their subquotients) may have invariants under the action of the unit groups $V_{N,+}$ (or, more generally, the isotropy groups $\Gamma_{\mathcal{C}, U^{\prime}}$ ), so that $q$-expansions of meromorphic differentials on $Y_{U, R}$ (and forms of 
weight $\left.\left(2 \mathbf{e}_{\theta},-\mathbf{e}_{\theta}\right)\right)$ may have nonzero constant terms and the morphism analogous to (29) may fail to be an isomorphism. (Note that in this case the relevant base-change morphisms $\left(\iota_{*} \mathcal{F}\right)_{R} \rightarrow \iota_{R, *}\left(\mathcal{F}_{R}\right)$ fail to be surjective at the cusps.)

We can, however, simplify matters by placing ourselves in the situation when this fails in the extreme. Suppose then that $p^{n} R=0$ for some $n>0$ and that $N$ is sufficiently large that $\nu \equiv 1 \bmod p^{n} \mathcal{O}_{F}$ for all $\nu \in V_{N,+}$. Arguing exactly as above, we find that $\iota_{R, *}\left(\Omega_{Y_{U, R} / R}^{1}\right)$ is now a vector bundle over $Y_{U, R}^{\min }$ whose completion at $\mathcal{C}$ is identified with $\left(M \otimes R\left[\left[q^{m}\right]\right]_{m \in N^{-1} M_{+} \cup\{0\}}\right)^{V_{N,+}}=M \otimes \mathcal{O}_{Y_{U, R}^{\min }, \mathcal{C}}^{\wedge}$

$$
=\left\{\sum_{m \in N^{-1} M_{+} \cup\{0\}} c_{m} \otimes q^{m} \mid c_{\nu m}=\nu c_{m} \forall \nu \in V_{N,+}, m \in N^{-1} M_{+}\right\},
$$

with the canonical derivation given by $\sum r_{m} q^{m} \mapsto \sum m \otimes r_{m} q^{m}$. Furthermore, each

$$
\operatorname{Fil}^{j}\left(\iota_{R, *}\left(\Omega_{Y_{U, R} / R}^{1}\right)_{\mathfrak{p}, i}\right)=\iota_{R, *}\left(\operatorname{Fil}^{j}\left(\Omega_{Y_{U, R} / R}^{1}\right)_{\mathfrak{p}, i}\right)
$$

is a subbundle whose completion at $\mathcal{C}$ is identified with $t_{j}(M \otimes \mathcal{O})_{\tau} \otimes_{\mathcal{O}} \mathcal{O}_{Y_{U, R}^{\min , \mathcal{C}}}^{\wedge}$ and the natural maps

$$
\operatorname{gr}^{j}\left(\iota_{R, *}\left(\Omega_{Y_{U, R} / R}^{1}\right)_{\mathfrak{p}, i}\right) \hookrightarrow \iota_{R, *}\left(\operatorname{gr}^{j}\left(\Omega_{Y_{U, R} / R}^{1}\right)_{\mathfrak{p}, i}\right) \stackrel{\sim}{\longrightarrow} \iota_{R, *} \mathcal{A}_{2 \mathbf{e}_{\theta},-\mathbf{e}_{\theta}, R}
$$

are isomorphisms of line bundles over $Y_{U, R}^{\min }$ whose completions at the cusps are induced by the surjections $t_{j}(M \otimes \mathcal{O})_{\tau} \rightarrow M_{\theta}$. We remark also that this carries over with $U$ replaced by arbitrary $U^{\prime}$, provided $U^{\prime}$ is sufficiently small that (in additional to the usual hypotheses) $\alpha \equiv \delta \bmod p^{n} \mathcal{O}_{F}$ for all $\left(\begin{array}{cc}\alpha & \beta \\ 0 & \delta\end{array}\right) \in \Gamma_{\mathcal{C}, U^{\prime}}$ and cusps $\mathcal{C}^{\prime}$ of $Y_{U^{\prime}}$ (the condition being independent of the choice of $N$ and $\mathcal{C}$ in the definition of $\Gamma_{\mathcal{C}, U^{\prime}}$ ).

\section{Operators on $q$-expansions}

\subsection{Partial Hasse invariants}

We next describe the effect of the various weight-shifting operators on $q$-expansions, beginning with the simplest case of (multiplication by) partial Hasse invariants. We will now only be working in the setting of $R=\mathbb{F}$, and we will use $\cdot$ to denote base changes from $\mathcal{O}$ to $\mathbb{F}$. Since the formation of $q$-expansions is compatible in the obvious sense with pullback under the projections $\bar{Y}_{U} \rightarrow \bar{Y}_{U^{\prime}}$, it will suffice to consider the case $U=U(N)$.

Recall that in Subsection 4.1 we defined the partial Hasse invariants as certain elements

$$
H_{\theta} \in M_{\mathbf{h}_{\theta}, \mathbf{0}}(U ; \mathbb{F})=H^{0}\left(\bar{Y}_{U}, \overline{\mathcal{A}}_{\mathbf{h}_{\theta}, \mathbf{0}}\right)
$$

where $\mathbf{h}_{\theta}:=n_{\theta} \mathbf{e}_{\sigma^{-1} \theta}-\mathbf{e}_{\theta}$, with $n_{\theta}=p$ if $j=1$ and $n_{\theta}=1$ if $j>1$. In particular, if $j>1$, then $H_{\theta}$ is defined by the morphism $u: \mathcal{L}_{\tau, j} \rightarrow \mathcal{L}_{\tau, j-1}$ induced by $\varpi_{\mathfrak{p}}$ on the universal abelian variety over $\widetilde{Y}_{U, \mathbb{F}}$, which evidently extends to the endomorphism $\varpi_{\mathfrak{p}}$ of $A^{\text {tor }}$ over 
$S:=\widetilde{Y}_{U, \mathbb{F}}^{\text {tor }}$. Since its pullback via $\bar{\xi}$ is defined by $\varpi_{\mathfrak{p}}$ on $\bar{T}_{I, J}$, the resulting morphism of line bundles

$$
\bar{\xi}^{*} \mathcal{L}_{\tau, j}^{\text {tor }} \longrightarrow \bar{\xi}^{*} \mathcal{L}_{\tau, j-1}^{\text {tor }}
$$

is compatible with their canonical trivialisations and, more precisely, with the morphism

$$
\left(I^{-1}\right)_{\theta} \otimes_{\mathcal{O}} \mathbb{F} \rightarrow\left(I^{-1}\right)_{\sigma^{-1} \theta} \otimes_{\mathcal{O}} \mathbb{F}
$$

induced by $u=\varpi \otimes 1$ on $\left(I^{-1} \otimes \mathcal{O}\right)_{\tau}$. It follows that $H_{\theta}$ has constant $q$-expansion, where the constant corresponds to the basis element $\varpi_{\mathfrak{p}}$ under the identification

$$
\bar{D}_{\mathbf{h}_{\theta}, \mathbf{0}} \cong \mathfrak{p} \otimes_{\mathcal{O}_{F}, \theta} \mathbb{F}
$$

provided by (26).

For $j=1$ we use also that the morphism of line bundles

$$
\bar{\xi}^{*} \mathcal{L}_{\tau, 1}^{\text {tor }} \longrightarrow \bar{\xi}^{*} \phi_{S}^{*}\left(\mathcal{L}_{\phi^{-1} \circ \tau, e_{\mathfrak{p}}}^{\text {tor }}\right)
$$

induced by the Verschiebung $\phi_{S}^{*} \bar{T}_{I, J} \rightarrow \bar{T}_{I, J}$ is compatible via the canonical trivialisations with the canonical isomorphism

$$
\left(I^{-1} \otimes \mathbb{F}\right)_{\tau} \stackrel{\sim}{\longrightarrow} \phi_{S}^{*}\left(I^{-1} \otimes \mathbb{F}\right)_{\phi^{-1} \circ \tau} .
$$

So in this case we again find that $H_{\theta}$ has constant $q$-expansion, the constant now corresponding to the basis element $\varpi_{\mathfrak{p}}^{1-e_{\mathfrak{p}}}$ under the identification

$$
\bar{D}_{\mathbf{h}_{\theta}, \mathbf{0}} \cong \mathfrak{p}^{1-e_{\mathfrak{p}}} \otimes_{\mathcal{O}_{F}, \theta} \mathbb{F}
$$

given by (26).

The $q$-expansions of the canonical sections $G_{\theta} \in M_{\mathbf{0}, \mathbf{h}_{\theta}}(U ; \mathbb{F})$ may be described similarly. Indeed, for $j>1$, the composites

$$
\begin{array}{ccccc}
\mathcal{L}_{\tau, j} & \longrightarrow & \mathcal{P}_{\tau, j} & \stackrel{u}{\longrightarrow} & \mathcal{L}_{\tau, j-1} \\
\text { and } \mathcal{M}_{\tau, j-1} & \longrightarrow & \mathcal{P}_{\tau, j} & \longrightarrow & \mathcal{M}_{\tau, j}
\end{array}
$$

on $\widetilde{Y}_{U, \mathbb{F}}^{\text {ord }}$ are isomorphisms whose tensor product defines $\mathcal{N}_{\theta} \cong \mathcal{N}_{\sigma^{-1} \theta}$. Its unique extension to $\widetilde{Y}_{U, \mathbb{F}}^{\text {tord }}$, and hence to $\tilde{Y}_{U, \mathbb{F}}^{\text {tor }}$, is therefore the isomorphism whose pullback via $\bar{\xi}$ is the tensor product of the isomorphisms defined on canonical trivialisations by $\left(I^{-1}\right)_{\theta} \otimes_{\mathcal{O}} \mathbb{F} \stackrel{u}{\rightarrow}$ $\left(I^{-1}\right)_{\sigma^{-1} \theta} \otimes \mathcal{O} \mathbb{F}$ and by the identity on $\left(\mathfrak{d} J^{-1} \otimes \mathbb{F}\right)_{\tau} / u\left(\mathfrak{d} J^{-1} \otimes \mathbb{F}\right)_{\tau}$ and hence corresponds to

$$
\left(\mathfrak{d}(I J)^{-1}\right)_{\theta} \otimes_{\mathcal{O}} \mathbb{F} \stackrel{u}{\rightarrow}\left(\mathfrak{d}(I J)^{-1}\right)_{\sigma^{-1} \theta} \otimes_{\mathcal{O}} \mathbb{F} .
$$

Therefore, $G_{\theta}$ has constant $q$-expansion, with constant corresponding to the basis element $\varpi_{\mathfrak{p}}$ under the identification

$$
\bar{D}_{\mathbf{0}, \mathbf{h}_{\theta}} \cong \mathfrak{p} \otimes \mathcal{O}_{F}, \theta \mathbb{F}
$$

provided by the analogue of (26) for $\left(\mathfrak{d}(I J)^{-1}\right)_{\theta}$. 
Similarly, if $j=1$, we find that $\mathcal{N}_{\tau, 1} \cong \phi_{S}^{*}\left(\mathcal{N}_{\phi^{-1} \circ \tau, e_{\mathfrak{p}}}\right)$ over $S=\widetilde{Y}_{U, \mathbb{F}}^{\text {ord }}$ is the tensor product of the isomorphism

$$
\mathcal{L}_{\tau, 1} \rightarrow \phi_{S}^{*}\left(\mathcal{L}_{\phi^{-1} \circ \tau, e_{\mathfrak{p}}}\right)
$$

defining the Hasse invariant and the isomorphism

$$
\phi_{S}^{*}\left(\mathcal{M}_{\phi^{-1} \circ \tau, e_{\mathfrak{p}}}\right) \rightarrow \mathcal{M}_{\tau, 1}
$$

induced by $\operatorname{Frob}_{A}$. The extensions to $\widetilde{Y}_{U, \mathbb{F}}^{\text {tord }}$ are again compatible with the canonical trivialisations, now corresponding to maps whose tensor product is the inverse of the isomorphism

$$
\left(\left(\mathfrak{d}(I J)^{-1}\right)_{\sigma^{-1} \theta} \otimes_{\mathcal{O}} \mathbb{F}\right)^{\otimes p} \stackrel{\sim}{\longrightarrow}\left(\mathfrak{d}(I J)^{-1}\right)_{\theta} \otimes_{\mathcal{O}} \mathbb{F}
$$

induced by $u^{e_{\mathfrak{p}}-1}$. So again $G_{\theta}$ has constant $q$-expansion, with constant corresponding to $\varpi_{\mathfrak{p}}^{1-e_{\mathfrak{p}}}$ under the identification

$$
\bar{D}_{\mathbf{0}, \mathbf{h}_{\theta}} \cong \mathfrak{p}^{1-e_{\mathfrak{p}}} \otimes_{\mathcal{O}_{F}, \theta} \mathbb{F}
$$

given by (26) for $\left(\mathfrak{d}(I J)^{-1}\right)_{\theta}$.

\subsection{Partial $\Theta$-operators}

We now compute the effect of $\Theta$-operators on $q$-expansions exactly as in [12]. Recall from Subsection 5.2 that for each $\tau_{0}=\tau_{\mathfrak{p}, i} \in \Sigma_{0}$, the associated partial $\Theta$-operator is a map

$$
\Theta_{\tau_{0}}: M_{\mathbf{k}, \mathbf{l}}(U ; \mathbb{F}) \rightarrow M_{\mathbf{k}^{\prime}, \mathbf{l}^{\prime}}(U ; \mathbb{F})
$$

where $\mathbf{k}^{\prime}=\mathbf{k}+n_{\theta_{0}} \mathbf{e}_{\sigma^{-1} \theta_{0}}+\mathbf{e}_{\theta_{0}}, \mathbf{l}^{\prime}=\mathbf{l}+\mathbf{e}_{\theta_{0}}$ and $\theta_{0}=\theta_{\mathfrak{p}, i, e_{\mathfrak{p}}}$. It is defined for all sufficiently small $U$ of level prime to $p$ and is Hecke-equivariant. In particular, it is compatible with restriction for $U \subset U^{\prime}$, so we may assume $U=U(N)$ for some $N$ sufficiently large that $\nu \equiv 1 \bmod p \mathcal{O}_{F}$ for all $\nu \in V_{N,+}$.

Recall from the proof of Theorem 5.2.1 that $\Theta_{\tau_{0}}$ is defined by a morphism $\mathcal{A}_{\mathbf{k}, \mathbf{l}, \mathbb{F}} \rightarrow$ $\mathcal{A}_{\mathbf{k}^{\prime}, \mathbf{l}^{\prime}, \mathbb{F}}$ given locally on sections by formula (12). Our assumptions on $U$ imply that

$$
\left(\iota_{\mathbb{F}, *} \mathcal{A}_{\mathbf{k}, \mathbf{1}, \mathbb{F}}\right)_{\mathcal{C}}^{\wedge}=D_{\mathbf{k}, \mathbf{1}, \mathbb{F}} \otimes_{\mathbb{F}} \cdot \mathcal{O}_{Y_{U, \mathbb{F}}^{\min }, \mathcal{C}}^{\wedge}
$$

is free of rank 1 over $\mathcal{O}_{Y_{U, \mathbb{F}}^{\min }, \mathcal{C}}^{\wedge}$ for all weights $\mathbf{k}, \mathbf{l}$ and cusps $\mathcal{C}$, so the completion at $\mathcal{C}$ of $\iota_{\mathbb{F}, *} \Theta_{\tau_{0}}$ is the map

$$
\left(\iota_{\mathbb{F}, *} \mathcal{A}_{\mathbf{k}, \mathbf{l}, \mathbb{F}}\right)_{\mathcal{C}}^{\wedge} \rightarrow\left(\iota_{\mathbb{F}, *} \mathcal{A}_{\mathbf{k}^{\prime}, \mathbf{l}^{\prime}, \mathbb{F}}\right)_{\mathcal{C}}^{\wedge}
$$

defined by (12), where $y_{\theta}$ is any basis for $\left(\iota_{\mathbb{F}, *} \mathcal{L}_{\theta}\right)_{\mathcal{C}}^{\wedge}$ and $y^{\mathbf{k}}=\prod_{\theta} y_{\theta}^{k_{\theta}}$. In particular, we may choose $y_{\theta}=b_{\theta} \otimes 1$ where $b_{\theta}$ is a basis for $D_{\mathbf{e}_{\theta}, \mathbf{0}, \mathbb{F}}=\left(I^{-1}\right)_{\theta} \otimes_{\mathcal{O}} \mathbb{F}$. The fact that $H_{\theta}$ has (nonzero) constant $q$-expansion at $\mathcal{C}$ then means the same holds for the element $r_{\theta} \in \mathcal{O}_{Y_{U, \mathbb{F}}^{\min }, \mathcal{C}}^{\wedge}$; that is, $r_{\theta} \in \mathbb{F}^{\times}$. We can even select the bases $b_{\theta}$ so that $r_{\theta}=1$ for all $\theta$ by choosing any basis $b_{\mathfrak{p}}$ for $\left(I_{\mathfrak{p}} \otimes \mathbb{F}\right)_{\mathfrak{p}, 0}$ over $\mathbb{F}[u] / u^{e_{\mathfrak{p}}}$ for each $\mathfrak{p}$, letting $b_{\mathfrak{p}, i}$ correspond to $\left(\phi^{i}\right)^{*}\left(b_{\mathfrak{p}}\right)$ under the canonical isomorphism $\left(I_{\mathfrak{p}} \otimes \mathbb{F}\right)_{\mathfrak{p}, i} \cong\left(\phi^{i}\right)^{*}\left(I_{\mathfrak{p}} \otimes \mathbb{F}\right)_{\mathfrak{p}, 0}$ and defining $b_{\mathfrak{p}, i, j}$ to be the image of $u^{e_{\mathfrak{p}}-j} b_{\mathfrak{p}, i}$ in $\left(I^{-1}\right)_{\theta} \otimes_{\mathcal{O}} \mathbb{F}$. 
Recall also that $g^{\mathbf{l}}=\prod_{\theta} g_{\theta}^{l_{\theta}}$ in (12), where each $g_{\theta}$ is a trivialisation of $\mathcal{N}_{\theta}$ over $Y_{U, \mathbb{F}}$. Therefore, $g_{\theta}$ trivialises $\iota_{\mathbb{F}, *} \mathcal{N}_{\theta}$ over $Y_{U, \mathbb{F}}^{\min }$, from which it follows that $g_{\theta}=c_{\theta} \otimes 1$ for some basis $c_{\theta}$ of $D_{\mathbf{0}, \mathbf{e}_{\theta}, \mathbb{F}}=\left(\mathfrak{d}(I J)^{-1}\right)_{\theta} \otimes_{\mathcal{O}} \mathbb{F}$. The formula (12) therefore takes the form

$$
\Theta_{\tau_{0}}\left(b^{\mathbf{k}} c^{\mathbf{l}} \otimes \varphi_{f}\right)=H_{\theta_{0}} b^{\mathbf{k}} c^{\mathbf{l}} \otimes K S_{\tau_{0}}\left(d \varphi_{f}\right)=b^{\mathbf{k}+\mathbf{h}_{\theta_{0}}} c^{\mathbf{l}} \otimes K S_{\tau_{0}}\left(d \varphi_{f}\right),
$$

for $\varphi_{f} \in \mathcal{O}_{Y_{U, \mathbb{F}}^{\min }, \mathcal{C}}^{\wedge}$. Finally, the descriptions in Subsection 7.3 of the canonical derivation, the Kodaira-Spencer filtration and the isomorphism (29) in terms of $q$-expansions yield the formula

$$
\Theta_{\tau_{0}}\left(\sum_{m \in N^{-1} M_{+} \cup\{0\}}\left(b^{\mathbf{k}} c^{\mathbf{l}} \otimes r_{m}\right) q^{m}\right)=\sum_{m \in N^{-1} M_{+}}\left(b^{\mathbf{k}+\mathbf{h}_{\theta_{0}}} c^{\mathbf{l}} \bar{\tau}_{0}(m) \otimes r_{m}\right) q^{m}
$$

where $\bar{\tau}_{0}$ is the canonical projection

$$
\begin{aligned}
N^{-1} M & \rightarrow\left(N^{-1} M \otimes \mathbb{F}\right)_{\tau_{0}}=(M \otimes \mathbb{F})_{\tau_{0}} \\
& \rightarrow M \otimes_{\mathcal{O}_{F}, \bar{\theta}_{0}} \mathbb{F}=\left(\mathfrak{d}^{-1} I^{-1} J\right) \otimes_{\mathcal{O}_{F}, \bar{\theta}_{0}} \mathbb{F}=D_{2 \mathbf{e}_{\theta_{0}},-\mathbf{e}_{\theta_{0}}, \mathbb{F}}
\end{aligned}
$$

(writing $\bar{\theta}_{0}$ for the composite of $\mathcal{O}_{F} \stackrel{\theta_{0}}{\rightarrow} \mathcal{O} \rightarrow \mathbb{F}$ ). As noted above, it follows that (30) holds with $U=U(N)$ replaced by any sufficiently small open compact $U^{\prime}$ of level prime to $p$ and $\mathcal{C}$ replaced by any cusp of $Y_{U^{\prime}}$. In this case, the $q$-expansions are necessarily invariant under the natural action of $\Gamma_{\mathcal{C}, U^{\prime}}$ (whose compatibility with (30) is a consequence of the construction but is straightforward to check directly).

We see immediately from (30) that the operators $\Theta_{\tau}$ for varying $\tau$ commute. We see also that

$$
\Theta_{\tau_{0}}^{p}(f)=\Theta_{\tau_{1}}(f) H_{\theta_{0}}^{p} H_{\theta_{1}} G_{\theta_{1}}^{-1} \prod_{j=1}^{e_{\mathfrak{p}}-1}\left(H_{\sigma^{j} \theta_{0}} G_{\sigma^{j} \theta_{0}}^{-1}\right)
$$

where $\tau_{1}=\tau_{0} \circ \phi=\tau_{\mathfrak{p}, i+1}$ and $\theta_{1}=\sigma^{e_{\mathfrak{p}}} \theta_{0}=\theta_{\mathfrak{p}, i+1, e_{\mathfrak{p}}}$. Indeed, this follows from (30) together with the fact that the $q$-expansions of

$$
H_{\tau_{1}}=\prod_{j=1}^{e_{\mathfrak{p}}} H_{\sigma^{j} \theta_{0}} \quad \text { and } \quad G_{\tau_{1}}=\prod_{j=1}^{e_{\mathfrak{p}}} G_{\sigma^{j} \theta_{0}}
$$

are constants given by the canonical isomorphisms $\left(\left(I^{-1}\right)_{\theta_{0}} \otimes_{\mathcal{O}} \mathbb{F}\right)^{\otimes p} \cong\left(I^{-1}\right)_{\theta_{1}} \otimes_{\mathcal{O}} \mathbb{F}$ and $\left(\left(\mathfrak{d}(I J)^{-1}\right)_{\theta_{0}} \otimes_{\mathcal{O}} \mathbb{F}\right)^{\otimes p} \cong\left(\mathfrak{d}(I J)^{-1}\right)_{\theta_{1}} \otimes_{\mathcal{O}} \mathbb{F}$, so we get a commutative diagram

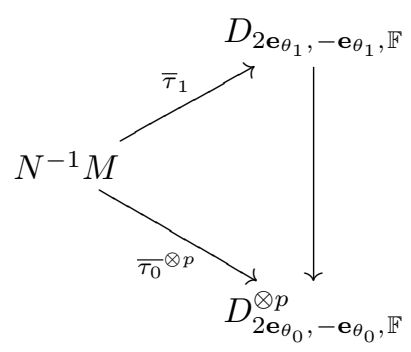

where the downward arrow is multiplication by the (constant) $q$-expansion of $H_{\tau_{1}}^{2} G_{\tau_{1}}^{-1}$. 


\subsection{Partial Frobenius operators}

Finally, we compute the effect on $q$-expansions of the partial Frobenius operators $V_{\mathfrak{p}}$ defined in Subsection 6.2. We must first extend the partial Frobenius endomorphisms $\widetilde{\Phi}_{\mathfrak{p}}$ (and $\Phi_{\mathfrak{p}}$ ) defined in Subsection 6.1 to compactifications. To that end, let $h_{\mathfrak{p}}$ denote the matrix $\left(\begin{array}{ll}1 & 0 \\ 0 & \delta\end{array}\right)$, viewed as an element of $\mathrm{GL}_{2}\left(\mathbb{A}_{F, \mathbf{f}}^{(p)}\right)$, where $\delta \in F^{\times}$is as in the definition fo $\widetilde{\Phi}_{\mathfrak{p}}$. We let $\widetilde{\Phi}_{\mathfrak{p}}^{\infty}: \widetilde{Y}_{U}^{\infty} \rightarrow \widetilde{Y}_{U}^{\infty}$ be the permutation defined on double cosets by $B_{1}\left(\mathcal{O}_{F,(p)}\right) g U^{p} \mapsto B_{1}\left(\mathcal{O}_{F,(p)}\right) h_{\mathfrak{p}}^{-1} g U^{p}$ and, similarly, let $\Phi_{\mathfrak{p}}^{\infty}$ denote the induced permutation of $Y_{U}^{\infty}$. Then $\widetilde{\Phi}_{\mathfrak{p}}^{\infty}$ translates to the map on corresponding data sending $(H, I, \lambda,[\eta])$ to $\left(H^{\prime}, I^{\prime}, \lambda^{\prime},\left[\eta^{\prime}\right]\right)$, where

- $I^{\prime}=\mathfrak{p}^{-1} \otimes_{\mathcal{O}_{F}} I=\mathfrak{p}^{-1} I$,

- $H^{\prime}$ is the pushout of $H$ with respect to $I \rightarrow I^{\prime}$,

- $\lambda^{\prime}=\delta \otimes \lambda$ (identifying $\wedge_{\mathcal{O}_{F}}^{2} H^{\prime}=\mathfrak{p}^{-1} \otimes_{\mathcal{O}_{F}} \wedge_{\mathcal{O}_{F}}^{2} H$ ) and

- $\eta^{\prime}=\eta$ (identifying $\widehat{\mathcal{O}}_{F}^{(p)} \otimes_{\mathcal{O}_{F}} H^{\prime}=\widehat{\mathcal{O}}_{F}^{(p)} \otimes_{\mathcal{O}_{F}} H$ ).

Suppose now that $U=U(N)$ for some sufficiently large $N$. One then checks that the morphism $\widetilde{\Phi}_{\mathfrak{p}}: \widetilde{Y}_{U, \mathbb{F}} \rightarrow \widetilde{Y}_{U, \mathbb{F}}$ extends to a morphism $\widetilde{\Phi}_{\mathfrak{p}}^{\text {tor }}: \widetilde{Y}_{U, \mathbb{F}}^{\text {tor }} \rightarrow \widetilde{Y}_{U, \mathbb{F}}^{\text {tor }}$ (where the ${ }^{\prime}$ indicates the choice of cone decomposition need not be the same) under which the component corresponding to a cusp $\widetilde{\mathcal{C}}$ represented by $(H, I, \lambda,[\eta])$ is sent to the one corresponding to $\widetilde{\mathcal{C}}^{\prime}=\widetilde{\Phi}_{\mathfrak{p}}^{\infty}(\widetilde{\mathcal{C}})$ represented by $\left(H^{\prime}, I^{\prime}, \lambda^{\prime},\left[\eta^{\prime}\right]\right)$, and the resulting map on completions pulls back to a morphism $\widehat{S}_{\widetilde{\mathcal{C}, \mathbb{F}}}^{\prime} \rightarrow \widehat{S}_{\widetilde{\mathcal{C}}^{\prime}, \mathbb{F}}$ whose effect on global sections corresponds under the isomorphisms of (18) to the homomorphism induced by the canonical inclusion

$$
M^{\prime}:=\mathfrak{d}^{-1}\left(I^{\prime}\right)^{-1} J=\mathfrak{p d}^{-1} I^{-1} J \hookrightarrow \mathfrak{d}^{-1} I^{-1} J .
$$

Furthermore, the pullback of $A^{\text {tor }}=\left(\widetilde{\Phi}_{\mathfrak{p}}^{\text {tor }}\right)^{*} A^{\text {tor }}$ to $\widehat{S}_{\widetilde{\mathcal{C}}, \mathbb{F}}^{\prime}$ is identified with the Tate semiabelian variety $T_{\mathfrak{p}^{-1} I, J}$, and the isomorphisms defined in Subsection 6.2 relating the line bundles $\widetilde{\Phi}_{\mathfrak{p}}^{*} \mathcal{L}_{\theta}$ to $\mathcal{L}_{\theta}$ or $\mathcal{L}_{\sigma^{-1} \theta}$ extend to isomorphisms

$$
\left(\widetilde{\Phi}_{\mathfrak{p}}^{\text {tor }}\right)^{*} \mathcal{L}_{\theta}^{\text {tor }} \cong \mathcal{L}_{\theta}^{\text {tor' }} \text { for } \theta \notin \Sigma_{\mathfrak{p}}, \text { and }\left(\widetilde{\Phi}_{\mathfrak{p}}^{\text {tor }}\right)^{*} \mathcal{L}_{\theta}^{\text {tor }} \cong\left(\mathcal{L}_{\sigma^{-1} \theta}^{\text {tor }}\right)^{\otimes n_{\theta}} \text { for } \theta \in \Sigma_{\mathfrak{p}}
$$

over $\tilde{Y}_{U, \mathbb{F}}^{\text {tor }} \mathbb{F}^{\prime}$ whose pullbacks are compatible via their canonical trivialisations with isomorphisms induced by the canonical $\left(\mathcal{O}_{F} \otimes \mathbb{F}\right)_{\tau}$-equivariant maps

$$
\left(p I^{-1} \otimes \mathbb{F}\right) \longrightarrow\left(\mathfrak{p} I^{-1} \otimes \mathbb{F}\right)_{\tau} \longrightarrow\left(I^{-1} \otimes \mathbb{F}\right)_{\tau}
$$

More precisely, if $\tau \notin \Sigma_{\mathfrak{p}, 0}$, then the second map is an isomorphism identifying $\left(\mathfrak{p} I^{-1}\right)_{\theta} \otimes_{\mathcal{O}}$ $\mathbb{F}$ with $\left(I^{-1}\right)_{\theta} \otimes_{\mathcal{O}} \mathbb{F}$, and if $\tau=\tau_{\mathfrak{p}, i}$ and $\theta=\theta_{\mathfrak{p}, i, j}$, then this map also induces the desired isomorphisms

$$
\begin{aligned}
& \left(\mathfrak{p} I^{-1}\right)_{\theta} \otimes_{\mathcal{O}} \mathbb{F}=u^{e_{\mathfrak{p}}-j}\left(\mathfrak{p} I^{-1} \otimes \mathbb{F}\right)_{\tau} \otimes_{\mathbb{F}[u] / u^{e} \mathfrak{p}} \mathbb{F} \\
& \stackrel{\sim}{\longrightarrow} u^{e_{\mathfrak{p}}-j+1}\left(I^{-1} \otimes \mathbb{F}\right)_{\tau} \otimes_{\mathbb{F}[u] / u^{e \mathfrak{p}}} \mathbb{F}=\left(I^{-1}\right)_{\sigma^{-1} \theta} \otimes_{\mathcal{O}} \mathbb{F}
\end{aligned}
$$

for $j=2, \ldots, e_{\mathfrak{p}}$. On the other hand, if $\tau=\tau_{\mathfrak{p}, i}$ and $\theta=\theta_{\mathfrak{p}, i, 1}$, then the first map induces an isomorphism

$$
\left(p I^{-1} \otimes \mathbb{F}\right)_{\tau} \otimes_{\mathbb{F}[u] / u^{e_{\mathfrak{p}}}} \mathbb{F} \stackrel{\sim}{\longrightarrow} u^{e_{\mathfrak{p}}-j}\left(\mathfrak{p} I^{-1} \otimes \mathbb{F}\right)_{\tau} \otimes_{\mathbb{F}[u] / u^{e_{\mathfrak{p}}}} \mathbb{F}=\left(\mathfrak{p} I^{-1}\right)_{\theta} \otimes_{\mathcal{O}} \mathbb{F}
$$


whose composite with the ones induced by

$$
\phi^{*}\left(I^{-1} \otimes \mathbb{F}\right)_{\phi^{-1} \circ \tau} \stackrel{\sim}{\longrightarrow}\left(I^{-1} \otimes \mathbb{F}\right)_{\tau} \stackrel{p \otimes 1}{\longrightarrow}\left(p I^{-1} \otimes \mathbb{F}\right)_{\tau}
$$

yields the desired isomorphism

$$
\left(\left(I^{-1}\right)_{\sigma^{-1} \theta} \otimes_{\mathcal{O}} \mathbb{F}\right)^{\otimes p} \stackrel{\sim}{\longrightarrow}\left(I^{-1}\right)_{\theta} \otimes_{\mathcal{O}} \mathbb{F} .
$$

The relations between the line bundles $\widetilde{\Phi}_{\mathfrak{p}}^{*} \mathcal{N}_{\theta}$ and $\mathcal{N}_{\theta}$ or $\mathcal{N}_{\sigma^{-1} \theta}$ extend similarly over $\widetilde{Y}_{U, \mathbb{F}}^{\text {tor }}$, , so for $\mathbf{k}^{\prime \prime}, \mathbf{l}^{\prime \prime}$ as in (14) we obtain isomorphisms

$$
\left(\widetilde{\Phi}_{\mathfrak{p}}^{\text {tor }}\right)^{*} \widetilde{\mathcal{A}}_{\mathbf{k}, 1, \mathbb{F}}^{\text {tor }} \cong \widetilde{\mathcal{A}}_{\mathbf{k}^{\prime \prime}, \mathbf{l}^{\prime \prime}, \mathbb{F}}^{\text {tor }}
$$

whose pullbacks to $\widehat{S}_{\widetilde{\mathcal{C}}, \mathbb{F}}^{\prime}$ are compatible via their canonical trivialisations with the isomorphisms

$$
\bar{D}_{\mathbf{k}, \mathbf{l}}^{\prime} \cong \bar{D}_{\mathbf{k}^{\prime \prime}, \mathbf{l}^{\prime \prime}}
$$

obtained as the tensor products of the ones just defined (where $\bar{D}_{\mathbf{k}, \mathbf{l}}^{\prime}$ is associated to the data for the cusp $\widetilde{\mathcal{C}}^{\prime}$ and $\bar{D}_{\mathbf{k}^{\prime \prime}, \mathbf{1}^{\prime \prime}}$ to the data for $\widetilde{\mathcal{C}}$ ).

It follows from the above description of $\widetilde{\Phi}_{\mathfrak{p}}^{\text {tor }}$ that $\widetilde{\Phi}_{\mathfrak{p}}$ extends to the morphism $\widetilde{\Phi}_{\mathfrak{p}}^{\text {min }}$ : $\widetilde{Y}_{U, \mathbb{F}}^{\min } \rightarrow \widetilde{Y}_{U, \mathbb{F}}^{\min }$ restricting to $\widetilde{\Phi}_{\mathfrak{p}}^{\infty}$ on the set of cusps, with the induced maps on completed local rings ${ }^{15}$ being the restriction to $V_{N}^{2}$-invariants of the canonical inclusion

$$
\mathbb{F}\left[\left[q^{m}\right]\right]_{m \in\left(N^{-1} M^{\prime}\right)_{+} \cup\{0\}} \longrightarrow \mathbb{F}\left[\left[q^{m}\right]\right]_{m \in\left(N^{-1} M\right)_{+} \cup\{0\}},
$$

where $M=\mathfrak{d}^{-1} I^{-1} J$ and $M^{\prime}=\mathfrak{p} M$. Furthermore, the commutativity of the diagram

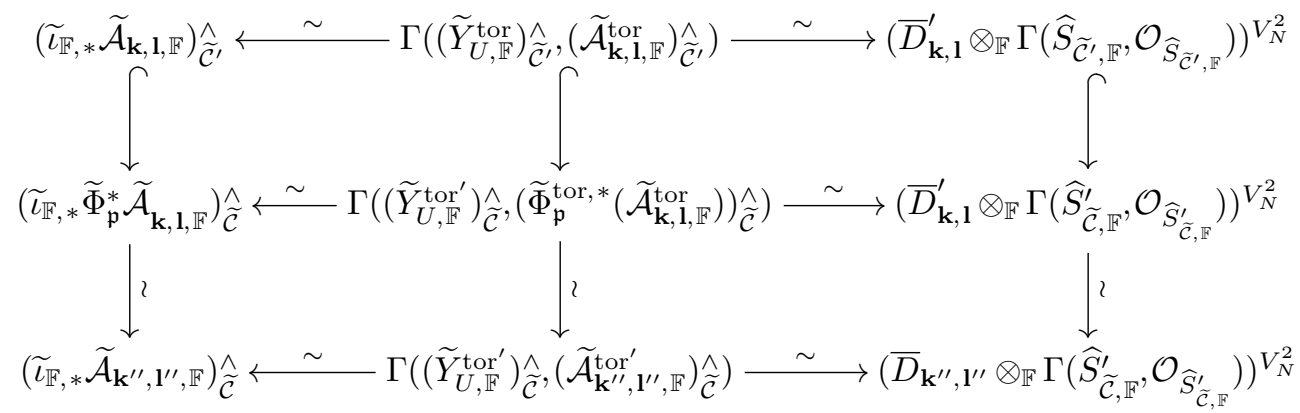

(where the top vertical arrows are defined by pulling back via $\widetilde{\Phi}_{\mathfrak{p}}^{\text {min }}, \widetilde{\Phi}_{\mathfrak{p}}^{\text {tor }}$ and the map $\left.\widehat{S}_{\widetilde{\mathcal{C}}, \mathbb{F}}^{\prime} \rightarrow \widehat{S}_{\widetilde{\mathcal{C}}^{\prime}, \mathbb{F}}\right)$ shows that the resulting map on $q$-expansions is the restriction to $V_{N^{-}}^{2}$ invariants of the map

$$
\bar{D}_{\mathbf{k}, \mathbf{l}}^{\prime} \otimes_{\mathbb{F}} \mathbb{F}\left[\left[q^{m}\right]\right]_{m \in\left(N^{-1} M^{\prime}\right)_{+} \cup\{0\}} \longrightarrow \bar{D}_{\mathbf{k}^{\prime \prime}, \mathbf{1}^{\prime \prime}} \otimes_{\mathbb{F}} \mathbb{F}\left[\left[q^{m}\right]\right]_{m \in\left(N^{-1} M\right)_{+} \cup\{0\}},
$$

${ }^{15}$ Note that we have implicitly chosen different representatives $(H, I, \lambda,[\eta])$ for each cusp $\widetilde{\mathcal{C}}$ according to whether $\widetilde{Y}_{U, \mathbb{F}}$ is viewed as the source or target of $\widetilde{\Phi}_{\mathfrak{p}}$, but the rings and modules arising from the two different descriptions of completions at $\widetilde{\mathcal{C}}$ are canonically isomorphic. 
obtained as the tensor product of (31) and (32). (Note that the isomorphism (31) is $V_{N}^{2}$-equivariant, but we can also choose $N$ sufficiently large that the action is trivial.)

The constructions above are all compatible with the natural action of $\mathcal{O}_{F,(p),+}^{\times}$, so the morphism $\widetilde{\Phi}_{\mathfrak{p}}^{\text {min }}$ induces a morphism $\Phi_{\mathfrak{p}}^{\min }: \bar{Y}_{U}^{\min } \rightarrow \bar{Y}_{U}^{\min }$ extending $\Phi_{\mathfrak{p}}$ by the map $\Phi_{\mathfrak{p}}^{\infty}$ on cusps, and its effect on completed local rings is given by the $V_{N,+}$-invariants of (32). Furthermore, the map $V_{\mathfrak{p}}: M_{\mathbf{k}, \mathbf{l}}(U ; \mathbb{F}) \longrightarrow M_{\mathbf{k}^{\prime \prime}, \mathbf{1}^{\prime \prime}}(U, \mathbb{F})$ is described on $q$-expansions by taking the $V_{N,+}$-invariants of the tensor product of (31) and (32). (Note that $\Phi_{\mathfrak{p}}^{\min }$ is proper and quasi-finite, and hence finite, but not necessarily flat at the cusps.)

Similarly, for any sufficiently small $U^{\prime}$, we may choose $N$ so $U=U(N) \subset U^{\prime}$ and take invariants under the natural action of $U^{\prime} / U$, with which the above constructions are also easily seen to be compatible. We thus obtain the description of $V_{\mathfrak{p}}$ on $q$-expansions (under the identifications of Proposition 7.2.1) as the resulting map

$$
\left(\bar{D}_{\mathbf{k}, \mathbf{l}}^{\prime} \otimes_{\mathbb{F}} \mathbb{F}\left[\left[q^{m}\right]\right]_{m \in\left(N^{-1} M^{\prime}\right)_{+} \cup\{0\}}\right)^{\Gamma_{\mathcal{C}^{\prime}, U^{\prime}}} \longrightarrow\left(\bar{D}_{\mathbf{k}^{\prime \prime}, \mathbf{1}^{\prime \prime}} \otimes_{\mathbb{F}} \mathbb{F}\left[\left[q^{m}\right]\right]_{m \in\left(N^{-1} M\right)_{+} \cup\{0\}}\right)^{\Gamma_{\mathcal{C}, U^{\prime} 8}} .
$$

(Note that the maps (31) and (32) are in fact $\Gamma_{\mathcal{C}}$-equivariant, where $\Gamma_{\mathcal{C}}$ is defined in (24) and its action on the target is via the natural inclusion in $\Gamma_{\mathcal{C}^{\prime}}$.)

Finally, we note that the effect of the operator $V_{\mathfrak{p}}^{0}: M_{\mathbf{k}, \mathbf{l}}(U ; \mathbb{F}) \longrightarrow M_{\mathbf{k}^{\prime \prime}, \mathbf{l}}(U, \mathbb{F})$ on $q-$ expansions has the same description but with (31) replaced by its composite with the isomorphism

$$
\bar{D}_{\mathbf{k}^{\prime \prime}, \mathbf{1}^{\prime \prime}} \cong \bar{D}_{\mathbf{0 , 1 - \mathbf { l } ^ { \prime \prime }}} \otimes_{\mathbb{F}} \bar{D}_{\mathbf{k}^{\prime \prime}, \mathbf{1}^{\prime \prime}}=\bar{D}_{\mathbf{k}^{\prime \prime}, \mathbf{l}}
$$

given by choosing the basis element of $\bar{D}_{\mathbf{0}, \mathbf{1}-\mathbf{1}^{\prime \prime}}$ to be the (constant) $q$-expansion of $\prod_{\theta \in \Sigma} G_{\theta}^{-l_{\theta}}$.

\section{The kernel of $\Theta$}

\subsection{Determination of the kernel}

In this section we analyze the kernel of the partial $\Theta$-operator $\Theta_{\tau}: M_{\mathbf{k}, \mathbf{l}}(U ; \mathbb{F}) \rightarrow$ $M_{\mathbf{k}^{\prime}, \mathbf{l}^{\prime}}(U ; \mathbb{F})$ for $\tau \in \Sigma_{\mathfrak{p}, 0}$ and relate it to the image of a partial Frobenius operator.

We allow $U$ to be any sufficiently small open compact subgroup of $\mathrm{GL}_{2}\left(\mathbb{A}_{F, \mathbf{f}}\right)$ of level prime to $p$ and $(\mathbf{k}, \mathbf{l})$ any weight such that $\chi_{\mathbf{k}+2 \mathbf{l}, \mathbb{F}}$ is trivial on $U \cap \mathcal{O}_{F}^{\times}$. First note that by (30) and the $q$-expansion principle (Proposition 7.2.2), the kernel of $\Theta_{\tau}$ consists precisely of those $f$ whose $q$-expansions $\sum_{m \in N^{-1} M_{+} \cup\{0\}}\left(b^{\mathbf{k}} c^{\mathbf{l}} \otimes r_{m}\right) q^{m}$ at all cusps, or even a cusp on each connected component, satisfy $r_{m}=0$ for all $m \notin \mathfrak{p} N^{-1} M$. (Recall that $M=\mathfrak{d}^{-1} I^{-1} J$ if the cusp $\mathcal{C}$ if represented by $(H, I,[\lambda],[\eta])$, and note that the condition depends only on the isomorphism class of $(H, I,[\lambda],[\eta])$ and, in particular, is independent of $N$ prime to $p$ such that $U(N) \subset U$.) Note that the condition is the same for all $\tau \in \Sigma_{\mathfrak{p}, 0}$, so that

$$
\operatorname{ker}\left(\Theta_{\tau}\right)=\operatorname{ker}\left(\Theta_{\tau^{\prime}}\right) \quad \text { for all } \tau, \tau^{\prime} \in \Sigma_{\mathfrak{p}, 0}
$$


Note also that the condition is invariant under multiplication by the Hasse invariants $H_{\theta}$ (and, of course, the forms $G_{\theta}$ ) for all $\theta$, so that $\Theta_{\tau}(f)=0$ if and only if

$$
\Theta_{\tau}\left(f \prod_{\theta \in \Sigma} G_{\theta}^{m_{\theta}} H_{\theta}^{n_{\theta}}\right)=0
$$

for all $\mathbf{m} \in \mathbb{Z}^{\Sigma}, \mathbf{n} \in \mathbb{Z}_{\geq 0}^{\Sigma}$, if and only if $\Theta_{\tau}\left(f \prod_{\theta \in \Sigma} G_{\theta}^{m_{\theta}} H_{\theta}^{n_{\theta}}\right)=0$ for some $\mathbf{m} \in \mathbb{Z}^{\Sigma}, \mathbf{n} \in \mathbb{Z}_{\geq 0}^{\Sigma}$. (Alternatively, note that this follows from the fact the partial $\Theta$-operators commute with multiplication by the $G_{\theta}$ and $H_{\theta}$, as can be seen directly from their definition.)

Suppose now that $\mathbf{k}=\mathbf{k}_{\min }(f)$, so that $f$ is not divisible by any partial Hasse invariants (see Subsection 4.2). Then if $f \in \operatorname{ker}\left(\Theta_{\tau}\right)$, and hence $f \in \operatorname{ker}\left(\Theta_{\tau_{\mathfrak{p}, i}}\right)$ for all $i \in \mathbb{Z} / f_{\mathfrak{p}} \mathbb{Z}$, then Theorem 5.2.1 implies that $p \mid k_{\theta_{\mathfrak{p}, i, e_{\mathfrak{p}}}}$ for all $i \in \mathbb{Z} / f_{\mathfrak{p}} \mathbb{Z}$. Therefore, $\mathbf{k}$ is of the form $\mathbf{k}_{0}^{\prime \prime}$ for some $\mathbf{k}_{0}$, where $\mathbf{k}_{0}^{\prime \prime}$ is as in the definition of $V_{\mathfrak{p}}$ in Subsection 6.2 or, equivalently, $V_{\mathfrak{p}}^{0}$. Furthermore, it is immediate from the description of the effect of $V_{\mathfrak{p}}^{0}$ on $q$-expansions in (33) that its image is contained in the kernel of $\Theta_{\tau}$. We now use the method of $[12$, Thm. 9.8.2] to prove that the kernel is precisely the image of $V_{\mathfrak{p}}^{0}$.

Theorem 9.1.1. Suppose that $f \in M_{\mathbf{k}_{0}^{\prime \prime}, 1}(U ; \mathbb{F})$ and $\tau \in \Sigma_{\mathfrak{p}, 0}$. If $\Theta_{\tau}(f)=0$, then $f=V_{\mathfrak{p}}^{0}(g)$ for some $g \in M_{\mathbf{k}_{0}, \mathbf{l}}(U ; \mathbb{F})$.

Proof. Let $\bar{\iota}$ denote the embedding $\bar{Y}_{U} \hookrightarrow \bar{Y}_{U}^{\min }$ and choose a set of cusps $\mathcal{S} \subset Y_{U}^{\infty}$ consisting of precisely one on each connected component of $\bar{Y}_{U}$. Note that since $\Phi_{\mathfrak{p}}^{\min }$ (defined in Subsection 8.3) is bijective on cusps as well as connected components, the set $\mathcal{S}^{\prime}:=\Phi_{\mathfrak{p}}^{\infty}(\mathcal{S})$ also includes exactly one cusp on each connected component.

Recall from Proposition 7.2.1 that the sheaves $\bar{\iota}_{*} \mathcal{A}_{\mathbf{k}_{0}, \mathbf{l}, \mathbb{F}}$ and $\bar{\iota}_{*} \mathcal{A}_{\mathbf{k}_{0}^{\prime \prime}, \mathbf{l}, \mathbb{F}}$ are coherent, as is $\bar{\iota}_{*} \Phi_{\mathfrak{p}, *} \mathcal{A}_{\mathbf{k}_{0}^{\prime \prime}, \mathbf{l}^{\prime \prime}, \mathbb{F}}=\Phi_{\mathfrak{p}, *}^{\min } \bar{\iota}_{*} \mathcal{A}_{\mathbf{k}_{0}^{\prime \prime}, \mathbf{l}, \mathbb{F}}$ since $\Phi_{\mathfrak{p}}^{\text {min }}$ is finite. For each $\mathcal{C} \in \mathcal{S}$, let $\mathcal{C}^{\prime}=\Phi_{\mathfrak{p}}^{\infty}(\mathcal{C})$, so that $\Phi_{\mathfrak{p}}^{\text {min,* }}$ defines a finite extension $\mathcal{O}_{\bar{Y}_{U}^{\min }, \mathcal{C}^{\prime}} \hookrightarrow \mathcal{O}_{\bar{Y}_{U}^{\min }, \mathcal{C}}$ of local rings. We let $N_{\mathcal{C}^{\prime}}=$ $\left(\bar{\iota}_{*} \mathcal{A}_{\mathbf{k}_{0}, \mathbf{l}, \mathbb{F}}\right)_{\mathcal{C}^{\prime}}$ denote the stalk at $\mathcal{C}^{\prime}$ of $\bar{\iota}_{*} \mathcal{A}_{\mathbf{k}_{0}, \mathbf{l}, \mathbb{F}}$ and, similarly, let $N_{\mathcal{C}}^{\prime \prime}=\left(\bar{\iota}_{*} \mathcal{A}_{\mathbf{k}_{0}, \mathbf{l}, \mathbb{F}}\right)_{\mathcal{C}}=$ $\left(\bar{\iota}_{*} \Phi_{\mathfrak{p}, *} \mathcal{A}_{\mathbf{k}_{0}^{\prime \prime}, \mathbf{l}, \mathbb{F}}\right)_{\mathcal{C}^{\prime}}$. The stalk at $\mathcal{C}^{\prime}$ of $\bar{\iota}_{*}$ of the adjoint of $\Phi_{\mathfrak{p}}^{*} \mathcal{A}_{\mathbf{k}_{0}, \mathbf{l}, \mathbb{F}} \stackrel{\sim}{\longrightarrow} \mathcal{A}_{\mathbf{k}_{0}^{\prime \prime}, \mathbf{l}, \mathbb{F}}$ then defines an injective homomorphism $N_{\mathcal{C}^{\prime}} \rightarrow N_{\mathcal{C}}^{\prime \prime}$ of finitely generated $\mathcal{O}_{\bar{Y}_{U}{ }^{\min },^{\prime}}$-modules, extending $V_{\mathfrak{p}}^{0}$ to a map

$$
\bigoplus_{\mathcal{C}^{\prime} \in \mathcal{S}^{\prime}} N_{\mathcal{C}^{\prime}} \rightarrow \bigoplus_{\mathcal{C} \in \mathcal{S}} N_{\mathcal{C}}^{\prime \prime}
$$

Similarly, localising at the generic points of $\bar{Y}_{U}$ (or, equivalently, $\bar{Y}_{U}^{\text {min }}$ ) extends $V_{\mathfrak{p}}^{0}$ to a map $H^{0}\left(\bar{Y}_{U}, \mathcal{A}_{\mathbf{k}_{0}, \mathbf{l}, \mathbb{F}} \otimes_{\bar{Y}_{U}} \mathcal{F}_{U}\right) \rightarrow H^{0}\left(\bar{Y}_{U}, \mathcal{A}_{\mathbf{k}_{0}^{\prime \prime}, \mathbf{l}, \mathbb{F}} \otimes_{\bar{Y}_{U}} \mathcal{F}_{U}\right)$, so we obtain a commutative diagram of injective maps

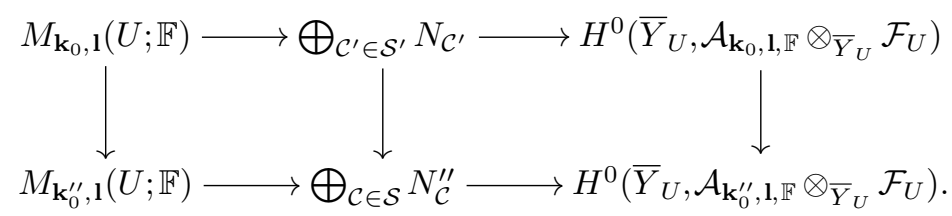

(Note that the horizontal maps, defined by localisation, are injective since $\mathcal{S}$ and $\mathcal{S}^{\prime}$ each contain a unique cusp on each component of $\bar{Y}_{U}$.) 
Let $N_{\mathcal{C}^{\prime}}^{\wedge}$ denote the completion of $N_{\mathcal{C}^{\prime}}$ with respect to the maximal ideal of $\mathcal{O}_{\bar{Y}_{U}^{\text {min }}, \mathcal{C}^{\prime}}$, and, similarly, let $N_{\mathcal{C}}^{\prime \prime \wedge}$ denote the completion of $N_{\mathcal{C}}^{\prime \prime}$ with respect to the maximal ideal of $\mathcal{O}_{\bar{Y}_{U}^{\min }, \mathcal{C}}$ or, equivalently, $\mathcal{O}_{\bar{Y}_{U}^{\min }, \mathcal{C}^{\prime}}$. Note that the map $N_{\mathcal{C}^{\prime}}^{\wedge} \rightarrow N_{\mathcal{C}}^{\prime \prime \wedge}$ is the one described by (33) or, more precisely, its variant for $V_{\mathfrak{p}}^{0}$.

Recall from (30) that if $f \in \operatorname{ker} \Theta_{\tau}$, then for each $\mathcal{C} \in \mathcal{S}$, the $q$-expansion of $f$,

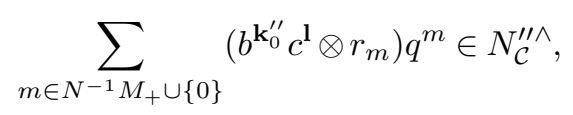

satisfies $r_{m}=0$ for all $m \notin \mathfrak{p} N^{-1} M=N^{-1} M_{+}^{\prime}$, where $M^{\prime}=\mathfrak{p} M$, and is therefore in the image of $N_{\mathcal{C}^{\prime}}^{\wedge}$. Since the completion $\mathcal{O}_{\bar{Y}_{U}^{\text {min }}, \mathcal{C}^{\prime}}^{\wedge}$ is faithfully flat over $\mathcal{O}_{\bar{Y}_{U}^{\text {min }}, \mathcal{C}^{\prime}}$, it follows that the image of $f$ in $\bigoplus_{\mathcal{C} \in \mathcal{S}} N_{\mathcal{C}}^{\prime \prime}$ is of the form $V_{\mathfrak{p}}^{0}(g)$ for some $g \in \bigoplus_{\mathcal{C}^{\prime} \in \mathcal{S}^{\prime}} N_{\mathcal{C}^{\prime}}$ and hence that its image in $H^{0}\left(\bar{Y}_{U}, \mathcal{A}_{\mathbf{k}_{0}^{\prime \prime}, \mathbf{l}, \mathbb{F}} \otimes_{\bar{Y}_{U}} \mathcal{F}_{U}\right)$ is of the form $V_{\mathfrak{p}}^{0}(g)$ for some $g \in H^{0}\left(\bar{Y}_{U}, \mathcal{A}_{\mathbf{k}_{0}^{\prime \prime}, \mathbf{l}, \mathbb{F}} \otimes_{\bar{Y}_{U}}\right.$ $\left.\mathcal{F}_{U}\right)$

It just remains to prove that $g \in M_{\mathbf{k}_{0}, \mathbf{l}}(U ; \mathbb{F})$ or, equivalently, that $\operatorname{ord}_{z}(g) \geq 0$ for all prime divisors $z$ on $\bar{Y}_{U}$. To that end, note that the operators $V_{\mathfrak{p}^{\prime}}$ for all $\mathfrak{p}^{\prime} \in S_{p}$ and $\epsilon_{\mathbf{k}, \mathbf{l}}$ for all $\mathbf{k}, \mathbf{l} \in \mathbb{Z}^{\Sigma}$ (see Subsection 6.2)) similarly extend to maps on stalks at generic points satisfying (16), so that $V_{\mathfrak{p}}(g)=f \prod_{\theta \in \Sigma} G_{\theta}^{l_{\theta}}$ and

$$
g^{p}=\left(\epsilon_{p \mathbf{k}, p \mathbf{l}} \prod_{\mathfrak{p}^{\prime} \in S_{p}} V_{\mathfrak{q}}^{e_{\mathfrak{q}}}\right)(g)=\left(\delta_{p \mathbf{k}, p \mathbf{l}} \prod_{\mathfrak{p}^{\prime} \neq \mathfrak{p}} V_{\mathfrak{p}^{\prime}}^{e_{\mathfrak{p}}}\right) V_{\mathfrak{p}}^{e_{\mathfrak{p}}-1}(f) .
$$

Therefore, $p \cdot \operatorname{ord}_{z}(g) \geq 0$, and hence $\operatorname{ord}_{z}(g) \geq 0$.

For the following corollary, recall that $\Xi^{\mathrm{min}}$ is defined by (10) and that the main result of [10] states that if $f$ is a nonzero form in $M_{\mathbf{k}, \mathbf{l}}(U ; \mathbb{F})$, then $\mathbf{k}_{\min }(f) \in \Xi^{\min }$.

Corollary 9.1.2. Suppose that $f \in M_{\mathbf{k}, \mathbf{l}}(U ; \mathbb{F})$ and $\tau \in \Sigma_{\mathfrak{p}, 0}$. Then $\Theta_{\tau}(f)=0$ if and only if there exist $\mathbf{k}_{0} \in \Xi^{\mathrm{min}}, \mathbf{n} \in \mathbb{Z}_{\geq 0}^{\Sigma_{0}}$ and $g \in M_{\mathbf{k}_{0}, \mathbf{l}}(U ; \mathbb{F})$ such that $\mathbf{k}=\mathbf{k}_{0}^{\prime \prime}+\sum_{\theta} n_{\theta} \mathbf{h}_{\theta}$ and

$$
f=V_{\mathfrak{p}}^{0}(g) \prod_{\theta} H_{\theta}^{n_{\theta}} \text {. }
$$

Proof. We have already seen that if $f=V_{\mathfrak{p}}^{0}(g) \prod_{\theta} H_{\theta}^{n_{\theta}}$, then $\Theta_{\tau}(f)=0$.

For the converse, note that we may assume $\mathbf{k}=\mathbf{k}_{\min }(f)$, so that $\mathbf{k} \in \Xi^{\min }$ and $\mathbf{k}=\mathbf{k}_{0}^{\prime \prime}$ for some $\mathbf{k}_{0} \in \mathbb{Z}^{\Sigma}$. Therefore, the theorem implies that $f=V_{\mathfrak{p}}^{0}(g)$ for some $g \in M_{\mathbf{k}_{0}, \mathbf{l}}(U ; \mathbb{F})$. Finally, it is immediate from the definitions of $\Xi^{\text {min }}$ and $\mathbf{k}_{0}^{\prime \prime}$ that $\mathbf{k}_{0} \in \Xi^{\text {min }}$ if (and only if) $\mathbf{k}_{0}^{\prime \prime} \in \Xi^{\mathrm{min}}$.

\subsection{Forms of partial weight 0}

We now apply our results on partial $\Theta$-operators to prove a partial positivity result for minimal weights of Hilbert modular forms. Recall the main result of [10] proves that minimal weights $\mathbf{k}=\sum k_{\theta} \mathbf{e}_{\theta}$ of Hilbert modular forms necessarily lie in the cone $\Xi^{\text {min }}$, and hence satisfy $k_{\theta} \geq 0$ for all $\theta$, and that forms with $\mathbf{k}=\mathbf{0}$ are easily described by Proposition 3.2.2. We prove the following restriction on possible minimal weights $\mathbf{k}$ with $k_{\theta}=0$ for some (but not all) $\theta \in \Sigma$. 
Theorem 9.2.1. Suppose that $\mathfrak{p} \in S_{p}$ is such that $f_{\mathfrak{p}}>1$, or $e_{\mathfrak{p}}>1$ and $p>3$. Suppose that $f \in M_{\mathbf{k}, \mathbf{l}}(U ; \mathbb{F})$ is nonzero and $\mathbf{k}=\mathbf{k}_{\min }(f)$. If $k_{\theta}=0$ for some $\theta \in \Sigma_{\mathfrak{p}}$, then $\mathbf{k}=\mathbf{0}$.

Proof. Writing simply $f=f_{\mathfrak{p}}$ and $e=e_{\mathfrak{p}}$, note that the hypotheses mean that ef $>1$ and $p^{f}>3$. Choose any $\tau=\tau_{\mathfrak{p}, i} \in \Sigma_{\mathfrak{p}, 0}$ and let $\theta_{0}=\theta_{\mathfrak{p}, i, e}$. We will first prove that $\Theta_{\tau}(f)=0$.

Note that since $\mathbf{k} \in \Xi^{\mathrm{min}}$ and $k_{\theta}=0$ for some $\theta \in \Sigma_{\mathfrak{p}}$, we in fact have $k_{\theta}=0$ for all $\theta \in \Sigma_{\mathfrak{p}}$. In particular, $p \mid k_{\theta_{0}}$, so Theorem 5.2.1 implies that $H_{\theta_{0}} \mid \Theta_{\tau}(f)$. Therefore, if $\Theta_{\tau}(f) \neq 0$, then $\mathbf{k}_{\min }\left(\Theta_{\tau}(f)\right) \leq_{\mathrm{Ha}} \mathbf{k}+2 \mathbf{e}_{\theta_{0}}$; that is,

$$
\mathbf{k}+2 \mathbf{e}_{\theta_{0}}-\sum_{\theta \in \Sigma} m_{\theta} \mathbf{h}_{\theta} \in \Xi^{\min }
$$

for some integers $m_{\theta} \geq 0$. Letting $m_{r}=m_{\sigma^{r} \theta_{0}}$ for $r=1, \ldots$, ef, this implies that

$$
\begin{aligned}
& m_{1}-m_{2} \leq m_{2}-m_{3} \leq \cdots \leq m_{e-1}-m_{e} \leq m_{e}-p m_{e+1} \\
\leq & p\left(m_{e+1}-m_{e+2}\right) \leq p\left(m_{e+2}-m_{e+3}\right) \leq \cdots \leq p\left(m_{2 e-1}-m_{2 e}\right) \leq p\left(m_{2 e}-p m_{2 e+1}\right) \\
& \vdots \\
\leq & p^{f-1}\left(m_{(f-1) e+1}-m_{(f-1) e+2}\right) \leq \cdots \leq p^{f-1}\left(m_{e f-1}-m_{e f}\right) \leq p^{f-1}\left(2+m_{e f}-p m_{1}\right) \\
\leq & p^{f}\left(m_{1}-m_{2}\right)
\end{aligned}
$$

(with the obvious collapsing here and in subsequent inequalities if $e$ or $f=1$ ). In particular, all of the expressions in (35) are nonnegative, so we have

$$
m_{1} \geq m_{2} \geq \cdots m_{e} \geq p m_{e+1} \geq p m_{e+2} \geq \cdots \geq p^{f-1} m_{e f-1} \geq p^{f-1} m_{e f}
$$

and $2+m_{e f}-p m_{1} \geq 0$, which implies that $\left(p^{f}-1\right) m_{e f} \leq 2$. Since $p^{f}>3$, it follows that $m_{e f}=0$, so $p m_{1} \leq 2$, which implies that either $m_{1}=0$ or $m_{1}=1$ and $p=2$. If $m_{1}=0$, then $m_{r}=0$ for all $r$, which contradicts the final inequality in (35). On the other hand, if $m_{1}=1$ and $p=2$, then all of the expressions in (35) are zero, which in turn implies that $m_{1}=p^{f-1} m_{e f}$, which again yields a contradiction.

We have now shown that $\Theta_{\tau}(f)=0$. Note that $\mathbf{k}^{\prime \prime}=\mathbf{k}$ since $k_{\theta}=0$ for all $\theta \in \Sigma_{\mathfrak{p}}$, so Theorem 5.2.1 implies that $f=V_{\mathfrak{p}}^{0}\left(f_{1}\right)$ for some $f_{1} \in M_{\mathbf{k}, \mathbf{l}}(U ; \mathbb{F})$. We may therefore iterate the above argument to conclude that $f_{1}=V_{\mathfrak{p}}^{0}\left(f_{2}\right)$ for some $f_{2} \in M_{\mathbf{k}, \mathbf{l}}(U ; \mathbb{F})$ and, by induction, that for all $n \geq 1$, we have $f=\left(V_{\mathfrak{p}}^{0}\right)^{n}\left(f_{n}\right)$ for some $f_{n} \in M_{\mathbf{k}, \mathbf{l}}(U ; \mathbb{F})$. It follows that for all $n \geq 1$, the $q$-expansion of $f$ at every cusp of $\bar{Y}_{U}$ satisfies $r_{m}=0$ for all $m \notin \mathfrak{p}^{n} M$, so in fact the $q$-expansion of $f$ at every cusp is constant.

To prove that $\mathbf{k}=\mathbf{0}$, recall that $\Xi^{\mathrm{min}}$ is contained in the cone spanned by the partial Hasse invariants, so $\mathbf{k}=\sum_{\theta \in \Sigma} s_{\theta} \mathbf{h}_{\theta}$ for some $s_{\theta} \in \mathbb{Q}_{\geq 0}$. Furthermore, the denominators are divisors of $M=\operatorname{lcm}\left\{p^{f_{\mathfrak{q}}}-1 \mid \mathfrak{q} \in S_{p}\right\}$, so that $M \mathbf{k}=\sum m_{\theta} \mathbf{h}_{\theta}$ for some $m_{\theta} \in \mathbb{Z}_{\geq 0}$. Similarly, $M \mathbf{l}=\sum n_{\theta} \mathbf{h}_{\theta}$ for some $n_{\theta} \in \mathbb{Z}$. Since $f$ has constant $q$-expansions, so does $f^{M}$, and therefore

$$
f^{M}=h \prod_{\theta \in \Sigma}\left(H_{\theta}^{m_{\theta}} G_{\theta}^{n_{\theta}}\right)
$$

for some $h \in H^{0}\left(\bar{Y}_{U}, \mathcal{O}_{\bar{Y}_{U}}\right)$. 
For each $\theta \in \Sigma$, the assumption that $\mathbf{k}=\mathbf{k}_{\min }(f)$ means that $f$ is not divisible by $H_{\theta}$, so $\operatorname{ord}_{z}(f)=0$ for some irreducible component $z$ of $Z_{\theta}$. On the other hand, we have $M \operatorname{ord}_{z}(f)=\operatorname{ord}_{z}\left(f^{M}\right) \geq m_{\theta}$, so $m_{\theta}=0$. As this holds for all $\theta \in \Sigma$, we conclude that $\mathbf{k}=\mathbf{0}$.

\subsection{The kernel revisited}

Finally, we present a cleaner, but less explicit, variant of Corollary 9.1.2 describing the kernels of partial $\Theta$-operators.

We first record the effect of $V_{\mathfrak{p}}$ on the partial Hasse invariants $H_{\theta}$. For each prime $\mathfrak{p} \in S_{p}$, we let $\beta_{\mathfrak{p}}=p^{-1} \varpi_{\mathfrak{p}}^{e_{\mathfrak{p}}} \in \mathcal{O}_{F, \mathfrak{p}}^{\times}$. It is straightforward to check, directly from the definition of $V_{\mathfrak{p}}$ or from the description (33) of its effect on $q$-expansions (and those of the $H_{\theta}$ in Subsection 8.1), that if $\theta \notin \Sigma_{\mathfrak{p}}$, then $V_{\mathfrak{p}}\left(H_{\theta}\right)=H_{\theta}$, but if $\theta=\theta_{\mathfrak{p}, i, j}$, then

$$
V_{\mathfrak{p}}\left(H_{\theta}\right)= \begin{cases}H_{\sigma^{-1} \theta}^{p}, & \text { if } e_{\mathfrak{p}}=1 \\ \bar{\theta}\left(\beta_{\mathfrak{p}}\right)^{-1} H_{\sigma^{-1} \theta}^{p}, & \text { if } e_{\mathfrak{p}}>1 \text { and } j=1 \\ \bar{\theta}\left(\beta_{\mathfrak{p}}\right) H_{\sigma^{-1} \theta}, & \text { if } e_{\mathfrak{p}}>1 \text { and } j=2 \\ H_{\sigma^{-1} \theta}, & \text { otherwise. }\end{cases}
$$

Therefore, we define the modified partial Hasse invariant to be $H_{\theta}^{\prime}=\bar{\theta}\left(\beta_{\mathfrak{p}^{\prime}}\right) H_{\theta}$ if $\theta=\theta_{\mathfrak{p}^{\prime}, i, 1}$ for some $\mathfrak{p}^{\prime} \in S_{p}$ and $i \in \mathbb{Z} / f_{\mathfrak{p}^{\prime}} \mathbb{Z}$ and $H_{\theta}^{\prime}=H_{\theta}$ otherwise, so that

$$
V_{\mathfrak{p}}\left(H_{\theta}^{\prime}\right)= \begin{cases}H_{\sigma_{\theta}^{-1} \theta}^{\prime n_{\theta}} & \text { if } \theta \in \Sigma_{\mathfrak{p}, 0} \\ H_{\theta}^{\prime} & \text { if } \theta \notin \Sigma_{\mathfrak{p}, 0} .\end{cases}
$$

Similarly, letting $G_{\theta}^{\prime}=\bar{\theta}\left(\beta_{\mathfrak{p}^{\prime}}\right) G_{\theta}$ if $\theta=\theta_{\mathfrak{p}^{\prime}, i, 1}$ and $G_{\theta}^{\prime}=G_{\theta}$ otherwise, we have $V_{\mathfrak{p}}\left(G_{\theta}^{\prime}\right)=$ $G_{\sigma^{-1} \theta}^{n_{\theta}}$ if $\theta \in \Sigma_{\mathfrak{p}}$ and $V_{\mathfrak{p}}\left(G_{\theta}^{\prime}\right)=G_{\theta}$ if $\theta \notin \Sigma_{\mathfrak{p}}$.

Now for any sufficiently small $U$ of level prime to $p$, consider the $\mathbb{F}$-algebra

$$
M_{\mathrm{tot}}(U ; \mathbb{F})=\bigoplus_{\mathbf{k}, \mathbf{l} \in\left(\mathbb{Z}^{\Sigma}\right)^{2}} M_{\mathbf{k}, \mathbf{l}}(U ; \mathbb{F})
$$

of Hilbert modular forms of all weights and level $U$ (where we let $M_{\mathbf{k}, \mathbf{l}}(U ; \mathbb{F})=0$ if $\chi_{\mathbf{k}+2 \mathbf{l}, \mathbb{F}}$ is nontrivial on $U \cap \mathcal{O}_{F}^{\times}$). We may then consider $V_{\mathfrak{p}}$ (respectively $\Theta_{\tau}$ ) as an $\mathbb{F}$-algebra homomorphism (respectively $\mathbb{F}$-linear derivation) $M_{\text {tot }}(U ; \mathbb{F}) \rightarrow M_{\text {tot }}(U ; \mathbb{F})$ for any $\mathfrak{p} \in S_{p}$ and $\tau \in \Sigma_{\mathfrak{p}, 0}$. Furthermore, letting $\mathfrak{I}$ denote the ideal $\left\langle H_{\theta}^{\prime}-1, G_{\theta}^{\prime}-1\right\rangle_{\theta \in \Sigma}$ in $M_{\text {tot }}(U ; \mathbb{F})$ and $R_{U}=M^{\text {tot }}(U ; \mathbb{F}) / \mathfrak{I}$, we see that $V_{\mathfrak{p}}(\mathfrak{I}) \subset \mathfrak{I}$ and $\Theta_{\tau}(\mathfrak{I}) \subset \mathfrak{I}$, so we obtain an $\mathbb{F}$-algebra homomorphism $V_{\mathfrak{p}}$ and derivation $\Theta_{\tau}$ such that the composite

$$
R_{U} \stackrel{V_{\mathfrak{p}}}{\longrightarrow} R_{U} \stackrel{\Theta_{\tau}}{\longrightarrow} R_{U}
$$

is zero for any $\mathfrak{p} \in S_{p}, \tau \in \Sigma_{\mathfrak{p}, 0}$.

Let $\Lambda$ denote the subgroup $\bigoplus_{\theta \in \Sigma} \mathbb{Z} \mathbf{h}_{\theta}$ of $\mathbb{Z}^{\Sigma}=\bigoplus_{\theta \in \Sigma} \mathbb{Z} \mathbf{e}_{\theta}$, so $\Lambda$ is the image of the image of the endomorphism of $\mathbb{Z}^{\Sigma}$ defined by $\sum_{\theta} m_{\theta} \mathbf{e}_{\theta} \mapsto \sum_{\theta} m_{\theta} \mathbf{h}_{\theta}$. Writing $\mathbf{h}_{\theta}=\sum_{\theta^{\prime}} n_{\theta, \theta^{\prime}} \mathbf{e}_{\theta^{\prime}}$, it is straightforward to check that the matrix $\left(n_{\theta, \theta^{\prime}}\right)$ has determinant $\prod_{\mathfrak{p} \in S_{p}}\left(p^{f_{\mathfrak{p}}}-1\right)$, so this is the index of $\Lambda$ in $\mathbb{Z}^{\Sigma}$. On the other hand, let $\Psi$ denote the group of characters 
$\psi:\left(\mathcal{O}_{F} / \mathfrak{q}\right)^{\times}=\bigoplus_{\mathfrak{p} \in S_{p}}\left(\mathcal{O}_{F} / \mathfrak{p}\right)^{\times} \longrightarrow \mathbb{F}^{\times}$and consider the surjective homomorphism $\varrho:$ $\mathbb{Z}^{\Sigma} \rightarrow \Psi$ defined by

$$
\varrho(\mathbf{k})=\prod_{\theta \in \Sigma} \bar{\theta}^{k_{\theta}}=\bigoplus_{\mathfrak{p} \in S_{p}}\left(\prod_{\theta \in \Sigma_{\mathfrak{p}}} \bar{\theta}^{k_{\theta}}\right) .
$$

Note that $\varrho\left(\mathbf{h}_{\theta}\right)$ is trivial for all $\theta \in \Sigma$, so $\Lambda \subset \operatorname{ker}(\varrho)$. Since $\mathbb{Z}^{\Sigma} / \Lambda$ and $\Psi$ each have order $\prod_{\mathfrak{p} \in S_{p}}\left(p^{f_{\mathfrak{p}}}-1\right)$, it follows that

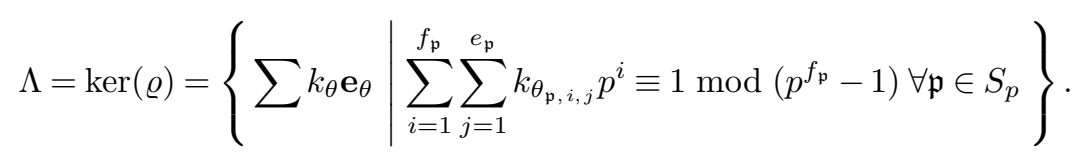

Remark 9.3.1. Recall that the $G_{\theta}$, and hence $G_{\theta}^{\prime}$, are invertible in $M^{\text {tot }}(U ; \mathbb{F})$, so if $\mathbf{l}^{\prime}$ $\mathbf{l}=\sum_{\theta} m_{\theta} \mathbf{h}_{\theta} \in \Lambda$, then multiplication by $\prod_{\theta} G_{\theta}^{\prime m_{\theta}}$ defines an isomorphism $M_{\mathbf{k}, \mathbf{l}}(U ; \mathbb{F}) \stackrel{\sim}{\rightarrow}$ $M_{\mathbf{k}, I^{\prime}}(U ; \mathbb{F})$. We may therefore write $R_{U}$ as the quotient of $\bigoplus_{\mathbf{k} \in \mathbb{Z}^{\Sigma}, \psi \in \Psi} M_{\mathbf{k}, \psi}(U ; \mathbb{F})$ by the ideal $\left\langle H_{\theta}^{\prime}-1\right\rangle_{\theta \in \Sigma}$, where $M_{\mathbf{k}, \varrho(1)}(U ; \mathbb{F})$ is canonically isomorphic to $M_{\mathbf{k}, 1}(U ; \mathbb{F})$ for each $l \in \mathbb{Z}^{\Sigma}$. Furthermore, the main result of [10] immediately implies that the natural map

$$
\bigoplus_{\mathbf{k} \in \Xi^{\min }, \psi \in \Psi} M_{\mathbf{k}, \psi}(U ; \mathbb{F}) \longrightarrow R_{U}
$$

is surjective, so we may also replace $\mathbb{Z}^{\Sigma}$ by the submonoid $\Xi^{\min }+\Lambda$ as the index set for $\mathbf{k}$ in the definition of $R_{U}$.

We will now describe the ideal $\mathfrak{I}$ in terms of $q$-expansions. For each cusp $\mathcal{C} \in Y_{U}^{\infty}$ we choose a representative $(H, I,[\lambda],[\eta])$, and for each $\mathbf{k}, \mathbf{l} \in \mathbb{Z}^{\Sigma}$, we let $\bar{D}_{\mathbf{k}, \mathbf{l}}^{\mathcal{C}}$ denote the one-dimensional vector space $\mathbb{F} \otimes_{\mathcal{O}} D_{\mathbf{k}, 1}$ over $\mathbb{F}$ (where $D_{\mathbf{k}, 1}$ is defined by (20)). We then let $\bar{D}_{\text {tot }}^{\mathcal{C}}$ denote $\bigoplus_{\mathbf{k}, \mathbf{l} \in \mathbb{Z}_{\Sigma}} \bar{D}_{\mathbf{k}, \mathbf{1}}$ with its natural $\mathbb{F}$-algebra structure. For each $\theta \in \Sigma$, let $c_{\theta} \in \bar{D}_{\mathbf{h}_{\theta}, \mathbf{0}}^{\mathcal{C}}$ denote the (constant) $q$-expansion of $H_{\theta}^{\prime}$ at $\mathcal{C}$. (Recall that the $q$-expansions of $H_{\theta}$ were explicitly described in Subsection 8.1, from which one gets a description of $c_{\theta}$ by multiplying by $\bar{\theta}\left(b_{\mathfrak{p}}\right)$ if $\theta=\theta_{\mathfrak{p}, i, 1}$.) Similarly, let $d_{\theta} \in \bar{D}_{\mathbf{0}, \mathbf{h}_{\theta}}^{\mathcal{C}}$ denote the (constant) $q$-expansion of $G_{\theta}^{\prime}$ at $\mathcal{C}$, and define $\mathfrak{I}^{\mathcal{C}}$ to be the ideal $\left\langle c_{\theta}-1, d_{\theta}-1\right\rangle_{\theta \in \Sigma}$ of $\bar{D}_{\text {tot }}^{\mathcal{C}}$. We may then view the quotient $\bar{D}_{\text {tot }}^{\mathcal{C}} / \mathfrak{I}^{\mathcal{C}}$ as the space of $\Lambda^{2}$-coinvariants of the free $\mathbb{F}\left[\left(\mathbb{Z}^{\Sigma}\right)^{2}\right]$-module $\bar{D}_{\text {tot }}^{\mathcal{C}}$ and decompose

$$
\bar{D}_{\text {tot }}^{\mathcal{C}} / \mathfrak{I}^{\mathcal{C}}=\bigoplus_{\chi, \psi \in \Psi} \bar{D}_{\chi, \psi}^{\mathcal{C}}
$$

so that the natural projection map $\bar{D}_{\mathbf{k}, 1}^{\mathcal{C}} \rightarrow \bar{D}_{\varrho(\mathbf{k}), \varrho(1)}^{\mathcal{C}}$ is an isomorphism for all $\mathbf{k}, \mathbf{l} \in \mathbb{Z}^{\Sigma}$. Now observe that the collection of $q$-expansion maps

$$
M_{\mathbf{k}, \mathbf{l}}(U ; \mathbb{F}) \rightarrow \bar{D}_{\mathbf{k}, 1}^{\mathcal{C}} \otimes_{\mathbb{F}} \mathbb{F}\left[\left[q^{m}\right]\right]_{m \in N^{-1}} M_{+}^{\mathcal{C}} \cup\{0\}
$$


(where $M^{\mathcal{C}}=\mathfrak{d}^{-1} I^{-1} J$ and $U(N) \subset U$ ) induces an $\mathbb{F}$-algebra homomorphism

$$
\bar{q}: M_{\mathrm{tot}}(U ; \mathbb{F}) \longrightarrow \bigoplus_{\mathcal{C} \in Y_{U}^{\infty}} \bigoplus_{\chi, \psi \in \Psi} \bar{D}_{\chi, \psi}^{\mathcal{C}} \otimes_{\mathbb{F}} \mathbb{F}\left[\left[q^{m}\right]\right]_{m \in N^{-1} M_{+}^{\mathcal{C}} \cup\{0\}}
$$

Lemma 9.3.2. The kernel of $\bar{q}$ is $\mathfrak{I}$.

Proof. The inclusion $\mathfrak{I} \subset \operatorname{ker}(\bar{q})$ is clear from the definitions.

Suppose then that $\bar{q}(f)=0$ and write $f=\sum_{\mathbf{k}, \mathbf{l} \in W} f_{\mathbf{k}, \mathbf{l}}$ for some finite subset $W$ of $\mathbb{Z}^{\Sigma}$. For each $\chi \in \Psi$, choose $\mathbf{k}_{\chi} \in \varrho^{-1}(\chi)$ sufficiently large that $\mathbf{k} \leq_{\text {Ha }} \mathbf{k}_{\chi}$ for all $\mathbf{k} \in$ $\varrho^{-1}(\chi) \cap W$. Thus, for each $\mathbf{k} \in W$, there is a unique $\mathbf{m}_{\mathbf{k}}=\sum_{\theta} m_{\mathbf{k}, \theta} \mathbf{e}_{\theta} \in \mathbb{Z}_{\geq 0}^{\Sigma}$ such that $\mathbf{k}_{\varrho(\mathbf{k})}=\mathbf{k}+\sum_{\theta} m_{\mathbf{k}, \theta} \mathbf{h}_{\theta}$. Now note that

$$
g:=\sum_{\mathbf{k}, \mathbf{l} \in W} H^{\prime \mathbf{m}_{\mathbf{k}}} G^{\prime \mathbf{m}_{\mathbf{l}}} f_{\mathbf{k}, \mathbf{l}} \in \bigoplus_{\chi, \psi \in \Psi} M_{\mathbf{k}_{\chi}, \mathbf{k}_{\psi}}(U ; \mathbb{F})
$$

and that $f-g \in \mathfrak{I}$ (where $H^{\prime \mathbf{m}_{\mathbf{k}}}=\prod_{\theta} H_{\theta}^{\prime m_{\mathbf{k}, \theta}}$ and $G^{\prime \mathbf{m}_{\mathbf{l}}}=\prod_{\theta} G_{\theta}^{\prime m_{\mathbf{1}, \theta}}$ ). Since $\mathfrak{I} \subset \operatorname{ker}(\bar{q})$, it follows that $\bar{q}(g)=0$. However, $\bar{q}$ restricts to the $q$-expansion map

$$
\bigoplus_{\chi, \psi \in \Psi} M_{\mathbf{k}_{\chi}, \mathbf{k}_{\psi}}(U ; \mathbb{F}) \longrightarrow \bigoplus_{\mathcal{C} \in Y_{U}^{\infty}} \bigoplus_{\chi, \psi \in \Psi} \bar{D}_{\chi, \psi}^{\mathcal{C}} \otimes_{\mathbb{F}} \mathbb{F}\left[\left[q^{m}\right]\right]_{m \in N^{-1} M_{+}^{\mathcal{c}} \cup\{0\}}
$$

which is injective by Proposition 7.2.2, so $g=0$, and hence $f=f-g \in \mathfrak{I}$.

We also extract the following observation from the proof of the lemma.

Lemma 9.3.3. If $W \subset\left(\mathbb{Z}^{\Sigma}\right)^{2}$ is such that $(\varrho, \varrho): W \rightarrow \Psi^{2}$ is injective, then

$$
\mathfrak{I} \cap \bigoplus_{(\mathbf{k}, \mathbf{l}) \in W} M_{\mathbf{k}, \mathbf{l}}(U ; \mathbb{F})=0
$$

We are now ready to interpret the description of the kernel of the partial $\Theta$-operator in terms of the algebra $R_{U}$.

Theorem 9.3.4. If $\mathfrak{p} \in S_{p}, \tau \in \Sigma_{\mathfrak{p}, 0}$ and $U$ is any sufficiently small open compact subgroup of $\mathrm{GL}_{2}\left(\mathbb{A}_{F, \mathbf{f}}\right)$ containing $\mathrm{GL}_{2}\left(\mathcal{O}_{F, p}\right)$, then the sequence

$$
0 \longrightarrow R_{U} \stackrel{V_{\mathfrak{p}}}{\longrightarrow} R_{U} \stackrel{\Theta_{\tau}}{\longrightarrow} R_{U}
$$

is exact. 
Proof. The injectivity of $V_{\mathfrak{p}}: R_{U} \rightarrow R_{U}$ follows ${ }^{16}$ from Lemma 9.3.2 and the commutativity of the diagram

$$
\begin{array}{ccc}
M_{\mathrm{tot}}(U ; \mathbb{F}) & \stackrel{\bar{q}^{\prime}}{\longrightarrow} \bigoplus_{\mathcal{C}^{\prime} \in Y_{U}^{\infty}} \bigoplus_{\chi, \psi \in \Psi} \bar{D}_{\chi, \psi}^{\mathcal{C}^{\prime}} \otimes_{\mathbb{F}} \mathbb{F}\left[\left[q^{m}\right]\right]_{m \in N^{-1}} M_{+}^{\mathcal{C}^{\prime}} \cup\{0\} \\
V_{\mathfrak{p}} \downarrow & \downarrow \\
M_{\mathrm{tot}}(U ; \mathbb{F}) \stackrel{\bar{q}}{\longrightarrow} \bigoplus_{\mathcal{C} \in Y_{U}^{\infty}} \bigoplus_{\chi, \psi \in \Psi} \bar{D}_{\chi, \psi}^{\mathcal{C}} \otimes_{\mathbb{F}} \mathbb{F}\left[\left[q^{m}\right]\right]_{m \in N^{-1} M_{+}^{\mathcal{C}} \cup\{0\}},
\end{array}
$$

where the right downward arrow is the direct sum over $(\mathcal{C}, \chi, \psi)$ of the tensor product of the isomorphism $\bar{D}_{\chi, \psi}^{\mathcal{C}^{\prime}} \rightarrow \bar{D}_{\chi, \psi}^{\mathcal{C}}$ induced by (31) and the injective $\mathbb{F}$-algebra homomorphism (32). (Note that $(\varrho(\mathbf{k}), \varrho(\mathbf{l}))=\left(\varrho\left(\mathbf{k}^{\prime \prime}\right), \varrho\left(\mathbf{l}^{\prime \prime}\right)\right)$, and recall that the data $(H, I,[\lambda],[\eta])$ representing each cusp of $Y_{U}^{\infty}$ were implicitly chosen differently for the source and target of $\Phi_{\mathfrak{p}}$ to simplify the resulting description of $V_{\mathfrak{p}}$ on $q$-expansions in Subsection 8.3.)

Since $\Theta_{\tau} \circ V_{\mathfrak{p}}=0$, it just remains to prove that $\operatorname{ker}\left(\Theta_{\tau}\right) \subset \operatorname{im}\left(V_{\mathfrak{p}}\right)$. Suppose then that $f \in$ $M_{\text {tot }}(U ; \mathbb{F})$ is such that $\Theta_{\tau}(f) \in \mathfrak{I}$. As in the proof of Lemma 9.3.2, write $f=\sum_{\mathbf{k}, \mathbf{l} \in W} f_{\mathbf{k}, \mathbf{l}}$ for some finite subset $W$ of $\mathbb{Z}^{\Sigma}$, and for each $\chi \in \Psi$ choose $\mathbf{k}_{\chi} \in \varrho^{-1}(\chi)$ so that $\mathbf{k} \leq_{\mathrm{Ha}} \mathbf{k}_{\chi}$ for all $\mathbf{k} \in \varrho^{-1}(\chi) \cap W$ and consider

$$
g:=\sum_{\mathbf{k}, \mathbf{l} \in W} H^{\prime \mathbf{m}_{\mathbf{k}}} G^{\prime \mathbf{m}_{\mathbf{l}}} f_{\mathbf{k}, \mathbf{l}} \in \bigoplus_{\chi, \psi \in \Psi} M_{\mathbf{k}_{\chi}, \mathbf{k}_{\psi}}(U ; \mathbb{F}) .
$$

Since $f-g \in \mathfrak{I}$ and $\Theta_{\tau}(f) \in \mathfrak{I}$, we have $\Theta_{\tau}(g) \in \mathfrak{I}$. Note, however, that

$$
\Theta_{\tau}(g) \in \bigoplus_{\chi, \psi \in \Psi} M_{\mathbf{k}_{\chi}^{\prime}, \mathbf{l}_{\psi}^{\prime}}(U ; \mathbb{F})
$$

where $\mathbf{k}_{\chi}^{\prime}=\mathbf{k}_{\chi}+n_{\theta_{0}} \mathbf{e}_{\sigma^{-1} \theta_{0}}+\mathbf{e}_{\theta_{0}}$ and $\mathbf{l}_{\psi}^{\prime}=\mathbf{k}_{\psi}-\mathbf{e}_{\theta_{0}}$, so Lemma 9.3.3 implies that $\Theta_{\tau}(g)=0$. Writing $g=\sum_{\chi, \psi} g_{\chi, \psi}$ with $g_{\chi, \psi} \in M_{\mathbf{k}_{\chi}, \mathbf{k}_{\psi}}$, this means that $\Theta_{\tau}\left(g_{\chi, \psi}\right)=0$ for all $\chi, \psi \in \Psi$.

Corollary 9.1.2 now implies that for each $\chi, \psi \in \Psi$, we have

$$
g_{\chi, \psi}=G^{\prime \mathbf{m}} H^{\prime \mathbf{n}} V_{\mathfrak{p}}\left(h_{\chi, \psi}\right)
$$

for some $h_{\chi, \psi} \in M_{\mathbf{k}_{0}, \mathbf{l}_{0}}(U ; \mathbb{F})$, where $\mathbf{k}_{0}, \mathbf{l}_{0}, \mathbf{m} \in \mathbb{Z}^{\Sigma}$ and $\mathbf{n} \in \mathbb{Z}_{\geq 0}^{\Sigma}$ depend on $\chi$ and $\psi$. It follows that $g_{\chi, \psi}-V_{\mathfrak{p}}\left(h_{\chi, \psi}\right) \in \mathfrak{I}$, so setting $h=\sum_{\chi, \psi} h_{\chi, \psi}$, we conclude that $f-V_{\mathfrak{p}}(h)=$ $(f-g)+\left(g-V_{\mathfrak{p}}(h)\right) \in \mathfrak{I}$, as required.

Finally, consider $R=\lim _{U} R_{U}$, where the direct limit is over all sufficiently small open compact subgroups $U$ containing $\mathrm{GL}_{2}\left(\mathcal{O}_{F, p}\right)$. (Note that this is the same as the quotient of

$$
M_{\text {tot }}(\mathbb{F}):=\underset{U}{\lim } M_{\text {tot }}(U ; \mathbb{F}) \cong \bigoplus_{\mathbf{k}, \mathbf{l} \in \mathbb{Z}^{\Sigma}}\left(\underset{\lim _{U}}{\longrightarrow} M_{\mathbf{k}, \mathbf{l}}(U ; \mathbb{F})\right)=\bigoplus_{\mathbf{k}, \mathbf{l} \in \mathbb{Z}^{\Sigma}} M_{\mathbf{k}, \mathbf{l}}(\mathbb{F})
$$

${ }^{16}$ Alternatively, one can appeal to Lemma 9.3.3 instead of Lemma 9.3.2 and argue similarly to the forthcoming proof of the other exactness assertion. 
by the ideal $\left\langle H_{\theta}^{\prime}-1, G_{\theta}^{\prime}-1\right\rangle_{\theta \in \Sigma}$.) Since the maps $V_{\mathfrak{p}}$ and $\Theta_{\tau}$ are Hecke-equivariant in the obvious sense, we obtain an $\mathbb{F}$-algebra endomorphism $V_{\mathfrak{p}}$ of $R$ and an $\mathbb{F}$-linear derivation $\Theta_{\tau}$ on $R$, each of which is $\mathrm{GL}_{2}\left(\mathbb{A}_{F, \mathbf{f}}^{(p)}\right)$-equivariant and such that the sequence

$$
0 \longrightarrow R \stackrel{V_{\mathfrak{p}}}{\longrightarrow} R \stackrel{\Theta_{\tau}}{\longrightarrow} R
$$

is exact.

Acknowledgements. The author thanks Payman Kassaei and Shu Sasaki for numerous helpful conversations, particularly in the context of collaboration on forthcoming work that motivates the results in this article. It is also a pleasure to acknowledge the evident debt this work owes to that of Reduzzi and Xiao in [32]. The author is also grateful to Deo, Dimitrov and Wiese, whose interest in partial $\Theta$-operators (and their kernels) encouraged him to explore the ramifications of the approach in [12] and to the referee for pointing out a number of typos and useful references.

Competing Interest. None.

\section{References}

[1] F. Andreatta and E. Z. Goren, Hilbert modular forms: $\bmod p$ and $p$-adic aspects, Mem. Amer. Math. Soc. 173(819) (2005), vi+100.

[2] P. Berthelot, L. Breen and W. Messing, Théorie de Dieudonné cristalline. II, Volume 930 of Lecture Notes in Mathematics (Springer-Verlag, Berlin, 1982).

[3] P. Berthelot and W. Messing, Théorie de Dieudonné cristalline. III. Théorèmes d'équivalence et de pleine fidélité, in The Grothendieck Festschrift, Vol. I. Vol. 86 of Progr. Math. (Birkhäuser, Boston, 1990), 173-247.

[4] C.-L. ChaI, Arithmetic minimal compactification of the Hilbert-Blumenthal moduli spaces, Ann. Math. (2) 131(3) (1990), 541-554.

[5] M. De Maria, Hilbert Modular Forms Modulo $p$ of Partial Weight One and Unramifiedness of Galois Representations, Ph.D. Thesis (University of Lille and University of Luxembourg, 2020).

[6] E. De Shalit And E. Z. Goren, A theta operator on Picard modular forms modulo an inert prime, Res. Math. Sci. 65 (2016) Paper No. 8, 65pp.

[7] E. De Shalit and E. Z. Goren, Theta operators on unitary Shimura varieties, Algebra Number Theory 13(8) (2019), 1829-1877.

[8] S. V. Deo, M. Dimitrov and G. Wiese, Unramifiedness of weight one Hilbert Hecke algebras, Preprint, 2019 available at https://arxiv.org/abs/1911.11196.

[9] F. Diamond and P. L. Kassaei, Minimal weights of Hilbert modular forms in characteristic p, Compos. Math. 153(9) (2017) 1769-1778,.

[10] F. Diamond and P. L. Kassaei, The cone of minimal weights for $\bmod p$ Hilbert modular forms, Preprint, 2020, https://arxiv.org/abs/2004.13227.

[11] F. Diamond, P. L. Kassaei and S. Sasaki, A mod $p$ Jacquet-Langlands relation and Serre filtration via the geometry of Hilbert modular varieties: Splicing and dicing, Astérisque, to appear.

[12] F. Diamond And S. SASAKi, A Serre weight conjecture for geometric Hilbert modular forms in characteristic $p$, Preprint, 2017, https://arxiv.org/abs/1712.03775.

[13] M. Dimitrov, Compactifications arithmétiques des variétés de Hilbert et formes modulaires de Hilbert pour $\Gamma_{1}(c, n)$, in Geometric Aspects of Dwork Theory, Vols. I, II (Walter de Gruyter, Berlin, 2004), 527-554. 
[14] B. Edixhoven, The weight in Serre's conjectures on modular forms, Invent. Math. 109(3) (1992), 563-594.

[15] E. Eischen, J. Fintzen, E. Mantovan and I. Varma, Differential operators and families of automorphic forms on unitary groups of arbitrary signature, Doc. Math. 23 (2018), 445-495.

[16] E. Eischen, A. Ghitza, J. Fintzen, E. Mantovan and A. McAndrew, Differential operators $\bmod p$ : analytic continuation and consequences, Algebra \& Number Theory, 15(6) (2021), 1469-1504 doi:10.2140/ant.2021.15.1469.

[17] E. Eischen And E. Mantovan, Entire theta operators at unramified primes, IMRN, (2021), doi:10.1093/imrn/rnab190.

[18] E. Z. Goren, Hasse invariants for Hilbert modular varieties, Israel J. Math. 122 (2001), $157-174$.

[19] E. Z. GoRen, Hilbert modular forms modulo $p^{m}$ : the unramified case, J. Number Theory 90(2) (2001), 341-375.

[20] B. H. Gross, A tameness criterion for Galois representations associated to modular forms $(\bmod$ p), Duke Math. J. 61(2) (1990), 445-517.

[21] N. Jochnowitz, Congruences between systems of eigenvalues of modular forms, Trans. Amer. Math. Soc. 270(1) (1982), 269-285.

[22] N. Jochnowitz, A study of the local components of the Hecke algebra mod l, Trans. Amer. Math. Soc. 270(1) (1982), 253-267.

[23] N. M. Katz, p-adic properties of modular schemes and modular forms, In Modular Functions of One Variable, III (Proc. Internat. Summer School, Univ. Antwerp, Antwerp, 1972), Lecture Notes in Mathematics, Vol. 350 (Springer, Berlin, 1973), 69-190.

[24] N. M. Katz, A result on modular forms in characteristic $p$, in Modular Functions of One Variable, V (Proc. Second Internat. Conf., Univ. Bonn, Bonn, 1976), Lecture Notes in Math., Vol. 601 (Springer, Berlin, 1977), 53-61.

[25] N. M. KATz, p-adic L-functions for CM fields, Invent. Math. 49(3) (1978), 199-297.

[26] T. Kimura And H. Nirtsuma, On Kunz's conjecture, J. Math. Soc. Japan 34(2) (1982), 371-378.

[27] K.-W. Lan, Arithmetic Compactifications of PEL-Type Shimura Varieties, Vol. 36 of London Mathematical Society Monographs Series (Princeton University Press, Princeton, NJ, 2013).

[28] K.-W. Lan, Higher Koecher's principle, Math. Res. Lett. 23(1) (2016), 163-199.

[29] G. PAPpas and M. RAPOport, Local models in the ramified case. II. Splitting models, Duke Math. J. 127(2) (2005), 193-250.

[30] S. Ramanujan, On certain arithmetical functions, in Collected Papers of Srinivasa Ramanujan (AMS Chelsea Publ., Providence, RI, 2000), 136-162.

[31] M. Rapoport, Compactifications de l'espace de modules de Hilbert-Blumenthal, Compos. Math. 36(3) (1978), 255-335.

[32] D. A. Reduzzi and L. XiaO, Partial Hasse invariants on splitting models of Hilbert modular varieties, Ann. Sci. Éc. Norm. Supér. (4) 50(3) (2017), 579-607.

[33] S. SASAKI, Integral models of Hilbert modular varieties in the ramified case, deformations of modular Galois representations, and weight one forms, Invent. Math. 215(1) (2019), $171-264$.

[34] J.-P. Serre, Congruences et formes modulaires [d'après H. P. F. Swinnerton-Dyer], in Séminaire Bourbaki, Lecture Notes in Math., Vol. 317 (Springer, Berlin, 1973), 319-338.

[35] I. Vollaard, On the Hilbert-Blumenthal moduli problem, J. Inst. Math. Jussieu 4(4) (2005), 653-683.

[36] T. Yamauchi, The weight reduction of $\bmod p$ Siegel modular forms for $\mathrm{GSp}_{4}$, Preprint, 2014, https://arxiv.org/abs/1410.7894. 